\title{
Asymptotic Distribution of Quasi-Normal Modes for Kerr-de Sitter Black Holes
}

\author{
Semyon Dyatlov
}

\begin{abstract}
We establish a Bohr-Sommerfeld type condition for quasinormal modes of a slowly rotating Kerr-de Sitter black hole, providing their full asymptotic description in any strip of fixed width. In particular, we observe a Zeeman-like splitting of the high multiplicity modes at $a=0$ (Schwarzschild-de Sitter), once spherical symmetry is broken. The numerical results presented in Appendix B show that the asymptotics are in fact accurate at very low energies and agree with the numerical results established by other methods in the physics literature. We also prove that solutions of the wave equation can be asymptotically expanded in terms of quasi-normal modes; this confirms the validity of the interpretation of their real parts as frequencies of oscillations, and imaginary parts as decay rates of gravitational waves.
\end{abstract}

Quasi-normal modes (QNMs) of black holes are a topic of continued interest in theoretical physics: from the classical interpretation as ringdown of gravitational waves [11] to the recent investigations in the context of string theory [33]. The ringdown ${ }^{1}$ plays a role in experimental projects aimed at the detection of gravitational waves, such as LIGO [1]. See [35] for an overview of the vast physics literature on the topic and $[6,36,37,53]$ for some more recent developments.

In this paper we consider the Kerr-de Sitter model of a rotating black hole and assume that the speed of rotation $a$ is small; for $a=0$, one gets the stationary Schwarzschild-de Sitter black hole. The de Sitter model corresponds to assuming that the cosmological constant $\Lambda$ is positive, which is consistent with the current Lambda-CDM standard model of cosmology.

\footnotetext{
${ }^{1}$ Here is an irresistible quote of Chandrasekhar "... we may expect that any initial perturbation will, during its last stages, decay in a manner characteristic of the black hole itself and independent of the cause. In other words, we may expect that during these last stages, the black hole emits gravitational waves with frequencies and rates of damping that are characteristic of the black hole itself, in the manner of a bell sounding its last dying notes."
} 
A rigorous definition of quasi-normal modes for Kerr-de Sitter black holes was given using the scattering resolvent in [26]. In Theorem 1 below we give an asymptotic description of QNMs in a band of any fixed width, that is, for any bounded decay rate. The result confirms the heuristic analogy with the Zeeman effect: the high multiplicity modes for the Schwarzschild black hole split.

Theorem 2 confirms the standard interpretation of QNMs as complex frequencies of exponentially decaying gravitational waves; namely, we show that the solutions of the scalar linear wave equation in the Kerr-de Sitter background can be expanded in terms of QNMs.

In the mathematics literature, quasi-normal modes of black holes were studied by Bachelot, Motet-Bachelot, and Pravica [3-5,39] using the methods of scattering theory. QNMs of Schwarzschild-de Sitter metric were then investigated by Sá Barreto-Zworski [41], resulting in the lattice of pseudopoles given by (0.3) below. For this case, Bony-Häfner [10] established polynomial cutoff resolvent estimates and a resonance expansion, Melrose-Sá Barreto-Vasy [38] obtained exponential decay for solutions to the wave equation up to the event horizons, and Dafermos-Rodnianski [17] used physical space methods to obtain decay of linear waves better than any power of $t$.

Quasi-normal modes for Kerr-de Sitter were rigorously defined in [26] and exponential decay beyond event horizons was proved in [27]. Vasy [50] has recently obtained a microlocal description of the scattering resolvent and in particular recovered the results of $[26,27]$ on meromorphy of the resolvent and exponential decay; see [50, Appendix] for how his work relates to [26]. The crucial component for obtaining exponential decay was the work of WunschZworski [52] on resolvent estimates for normally hyperbolic trapping.

We add that there have been many papers on decay of linear waves for Schwarzschild and Kerr black holes - see [2,9,18, 19, 22, 23, 28, 29,46, 47,49] and references given there. In that case the cosmological constant is 0 (unlike in the de Sitter case, where it is positive), and the methods of scattering theory are harder to apply because of an asymptotically Euclidean infinity.

Theorem 1. Fix the mass $M_{0}$ of the black hole and the cosmological constant

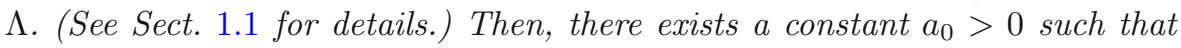
for $|a|<a_{0}$ and each $\nu_{0}$, there exist constants $C_{\omega}, C_{m}{ }^{2}$ such that the set of quasi-normal modes $\omega$ satisfying

$$
\operatorname{Re} \omega>C_{\omega}, \operatorname{Im} \omega>-\nu_{0}
$$

coincides modulo $O\left(|\omega|^{-\infty}\right)$ with the set of pseudopoles

$$
\omega=\mathcal{F}(m, l, k), m, l, k \in \mathbb{Z}, 0 \leq m \leq C_{m},|k| \leq l .
$$

(Since the set of QNMs is symmetric with respect to the imaginary axis, one also gets an asymptotic description for Re $\omega$ negative. Also, by [26, Theorem 4],

\footnotetext{
${ }^{2}$ As in [26], the indices $\omega, m, \ldots$ next to constants, symbols, operators, and functions do not imply differentiation.
} 
all QNMs lie in the lower half-plane). Here $\mathcal{F}$ is a complex valued classical symbol ${ }^{3}$ of order 1 in the $(l, k)$ variables, defined and smooth in the cone $\left\{m \in\left[0, C_{m}\right],|k| \leq l\right\} \subset \mathbb{R}^{3}$. The principal symbol $\mathcal{F}_{0}$ of $\mathcal{F}$ is real-valued and independent of $m$; moreover,

$$
\begin{gathered}
\mathcal{F}=\frac{\sqrt{1-9 \Lambda M_{0}^{2}}}{3 \sqrt{3} M_{0}}[(l+1 / 2)-i(m+1 / 2)]+O\left(l^{-1}\right) \quad \text { for } a=0 \\
\left(\partial_{k} \mathcal{F}_{0}\right)(m, \pm k, k)=\frac{\left(2+9 \Lambda M_{0}^{2}\right) a}{27 M_{0}^{2}}+O\left(a^{2}\right)
\end{gathered}
$$

The pseudopoles $(0.2)$ can be computed numerically; we have implemented this computation in a special case $l-|k|=O(1)$ and compared the pseudopoles with the QNMs computed by the authors of [6]. The results are described in Appendix B. One should note that the quantization condition of [41] was stated up to $O\left(l^{-1}\right)$ error, while Theorem 1 has error $O\left(l^{-\infty}\right)$; we demonstrate numerically that increasing the order of the quantization condition leads to a substantially better approximation.

Another difference between (0.2) and the quantization condition of [41] is the extra parameter $k$, resulting from the lack of spherical symmetry of the problem. In fact, for $a=0$ each pole in (0.3) has multiplicity $2 l+1$; for $a \neq 0$ this pole splits into $2 l+1$ distinct QNMs, each corresponding to its own value of $k$, the angular momentum with respect to the axis of rotation. (The resulting QNMs do not coincide for small values of $a$, as illustrated by (0.4)). In the physics literature this is considered an analog of the Zeeman effect.

Since the proof of Theorem 1 only uses microlocal analysis away from the event horizons, it implies estimates on the cutoff resolvent polynomial in $\omega$ (Proposition 1.3). Combining these with the detailed analysis away from the trapped set (and in particular near the event horizons) by Vasy [50], we obtain estimates on the resolvent on the whole space (Proposition 1.2). These in turn allow a contour deformation argument leading to an expansion of waves in terms of quasinormal modes. Such expansions have a long tradition in scattering theory going back to Lax-Phillips and Vainberg - see [45] for the strongly trapping case and for references.

For Schwarzschild-de Sitter black holes, a full expansion involving infinite sums over quasinormal modes was obtained in [10] (see also [13] for simpler expansions involving infinite sums over resonances). The next theorem presents an expansion of waves for Kerr-de Sitter black holes in the same style as the Bony-Häfner expansion:

Theorem 2. Under the assumptions of Theorem 1, take $\nu_{0}>0$ such that for some $\varepsilon>0$, every $Q N M \omega$ has $\left|\operatorname{Im} \omega+\nu_{0}\right|>\varepsilon$. (Such $\nu_{0}$ exists and can be chosen arbitrarily large, as the imaginary parts of QNMs lie within $O\left(|a|+l^{-1}\right)$ of those in (0.3)). Then, for s large enough depending on $\nu_{0}$, there exists a constant $C$ such that every solution $u$ to the Cauchy problem on the Kerr-de Sitter

\footnotetext{
${ }^{3}$ Here 'symbol' means a microlocal symbol as in for example [48, Section 8.1]. For the proofs, however, we will mostly use semiclassical symbols, as defined in Sect. 2.1.
} 
space

$$
\square_{g} u=0,\left.u\right|_{t^{*}=0}=f_{0} \in H^{s}\left(X_{\delta}\right),\left.\partial_{t^{*}} u\right|_{t^{*}=0}=f_{1} \in H^{s-1}\left(X_{\delta}\right),
$$

where $X_{\delta}=\left(r_{-}-\delta, r_{+}+\delta\right) \times \mathbb{S}^{2}$ is the space slice, $t^{*}$ is the time variable, and $\delta>0$ is a small constant (see Sect. 1.1 for details), satisfies for $t^{*}>0$,

$$
\left\|u\left(t^{*}\right)-\Pi_{\nu_{0}}\left(f_{0}, f_{1}\right)\left(t^{*}\right)\right\|_{H^{1}\left(X_{\delta}\right)} \leq C e^{-\nu_{0} t^{*}}\left(\left\|f_{0}\right\|_{H^{s}}+\left\|f_{1}\right\|_{H^{s-1}}\right) .
$$

Here

$$
\Pi_{\nu_{0}}\left(f_{0}, f_{1}\right)\left(t^{*}\right)=\sum_{\operatorname{Im} \widehat{\omega}>-\nu_{0}} e^{-i t^{*} \widehat{\omega}} \sum_{0 \leq j<J_{\widehat{\omega}}}\left(t^{*}\right)^{j} \Pi_{\widehat{\omega}, j}\left(f_{0}, f_{1}\right) ;
$$

the outer sum is over QNMs $\widehat{\omega}, J_{\widehat{\omega}}$ is the algebraic multiplicity of $\widehat{\omega}$ as a pole of the scattering resolvent, and $\Pi_{\widehat{\omega}, j}$ are finite rank operators mapping $H^{s}\left(X_{\delta}\right) \oplus H^{s-1}\left(X_{\delta}\right) \rightarrow C^{\infty}\left(X_{\delta}\right)$. Moreover, for $|\widehat{\omega}|$ large enough (that is, for all but a finite number of QNMs in the considered strip), $J_{\widehat{\omega}}=1, \Pi_{\widehat{\omega}, 0}$ has rank one, and

$$
\left\|\Pi_{\widehat{\omega}, 0}\right\|_{H^{s}\left(X_{\delta}\right) \oplus H^{s-1}\left(X_{\delta}\right) \rightarrow H^{1}\left(X_{\delta}\right)} \leq C|\widehat{\omega}|^{N-s} .
$$

Here $N$ is a constant depending on $\nu_{0}$, but not on $s$; therefore, the series (0.7) converges in $H^{1}$ for $s>N+2$.

The proofs start with the Teukolsky separation of variables used in [26], which reduces our problem to obtaining quantization conditions and resolvent estimates for certain radial and angular operators (Propositions 1.5 and 1.6). These conditions are stated and used to obtain Theorems 1 and 2 in Sect. 1. Also, at the end of Sect. 1.2 we present the separation argument in the simpler special case $a=0$, for convenience of the reader.

In the spherically symmetric case $a=0$, the angular problem is the eigenvalue problem for the Laplace-Beltrami operator on the round sphere. For $a \neq 0$, the angular operator $P_{\theta}$ is not self-adjoint; however, in the semiclassical scaling it is an operator of real principal type with completely integrable Hamiltonian flow. We can then use some of the methods of [31] to obtain a microlocal normal form for $h^{2} P_{\theta}$; since our perturbation is $O(h)$, we are able to avoid using analyticity of the coefficients of $P_{\theta}$. The quantization condition we get is global, similarly to [51]. The proof is contained in Sect. 3; it uses various tools from semiclassical analysis described in Sect. 2.

To complete the proof of the angular quantization condition, we need to extract information about the joint spectrum of $h^{2} P_{\theta}$ and $h D_{\varphi}$ from the microlocal normal form; for that, we formulate a Grushin problem for several commuting operators. The problem that needs to be overcome here is that existence of joint spectrum is only guaranteed by exact commutation of the original operators, while semiclassical methods always give $O\left(h^{\infty}\right)$ errors. This complication does not appear in $[31,32]$ as they study the spectrum of a single operator, nor in earlier works $[12,51]$ on joint spectrum of differential operators, as they use spectral theory of self-adjoint operators. Since this part of the construction can be formulated independently of the rest, we describe Grushin problems for several operators in an abstract setting in Appendix A. 
The radial problem is equivalent to one-dimensional semiclassical potential scattering. The principal part of the potential is real-valued and has a unique quadratic maximum; the proof of the quantization condition follows the methods developed in $[16,40,42]$. In [16], the microlocal behavior of the principal symbol near a hyperbolic critical point is studied in detail; however, only self-adjoint operators are considered and the phenomenon that gives rise to resonances in our case does not appear. The latter phenomenon is studied in [40] and [42]; our radial quantization condition, proved in Sect. 4, can be viewed as a consequence of [40, Theorems 2 and 4]. However, we do not compute the scattering matrix, which simplifies the calculations; we also avoid using analyticity of the potential near its maximum and formulate the quantization condition by means of real microlocal analysis instead of the action integral in the complex plane. As in [40], we use analyticity of the potential near infinity and the exact WKB method to relate the microlocal approximate solutions to the outgoing condition at infinity; however, the construction is somewhat simplified compared to [40, Sections 2 and 3] using the special form of the potential.

It would be interesting to see whether our statements still hold if one perturbs the metric, or if one drops the assumption of smallness of $a$. Near the event horizons, we rely on [26, Section 6], which uses a perturbation argument (thus smallness of $a$ ) and analyticity of the metric near the event horizons. Same applies to Sect. 4.2 of the present paper; the exact WKB construction there requires analyticity and Proposition 4.2 uses that the values $\omega_{ \pm}$defined in (4.9) are nonzero, which might not be true for large $a$. However, it is very possible that the construction of the scattering resolvent of [50] can be used instead. The methods of [50] are stable under rather general perturbations, see [50, Section 2.7], and apply in particular to Kerr-de Sitter black holes with $a$ satisfying $[50,(6.12)]$.

A more serious problem is the fact that Theorem 1 is a quantization condition, and thus is expected to hold only when the geodesic flow is completely integrable, at least on the trapped set. For large $a$, the separation of variables of Sect. 1.2 is still valid, and it is conceivable that the global structure of the angular integrable system in Sect. 3.2 and of the radial barrier-top Schrödinger operator in Sect. 4.1 would be preserved, yielding Theorem 1 in this case. Even then, the proof of Theorem 2 no longer applies as it relies on having gaps between the imaginary parts of resonances, which might disappear for large $a$.

However, a generic smooth perturbation of the metric supported near the trapped set will destroy complete integrability and thus any hope of obtaining Theorem 1. One way of dealing with this is to impose the condition that the geodesic flow is completely integrable on the trapped set. In principle, the global analysis of [51] together with the methods for handling $O(h)$ nonself-adjoint perturbations developed in Sect. 3 and Appendix A should provide the quantization condition in the direction of the trapped set, while the barrier-top resonance analysis of Sect. 4.3 should handle the transversal directions. However, without separation of variables one might need to merge these 
methods and construct a normal form at the trapped set which is not presented here.

Another possibility is to try to establish Theorem 2 without a quantization condition, perhaps under the (stable under perturbations) assumption that the trapped set is normally hyperbolic as in [52]. However, this will require to rethink the contour deformation argument, as it is not clear which contour to deform to when there is no stratification of resonances by depth, corresponding to the parameter $m$ in Theorem 1 .

\section{Proofs of Theorems 1 and 2}

\subsection{Kerr-de Sitter Metric}

First of all, we define Kerr-de Sitter metric and briefly review how solutions of the wave equation are related to the scattering resolvent; see also [26, Section 1] and $[50$, Section 6$]$. The metric is given by

$$
\begin{aligned}
g= & -\rho^{2}\left(\frac{d r^{2}}{\Delta_{r}}+\frac{d \theta^{2}}{\Delta_{\theta}}\right)-\frac{\Delta_{\theta} \sin ^{2} \theta}{(1+\alpha)^{2} \rho^{2}}\left(a d t-\left(r^{2}+a^{2}\right) d \varphi\right)^{2} \\
& +\frac{\Delta_{r}}{(1+\alpha)^{2} \rho^{2}}\left(d t-a \sin ^{2} \theta d \varphi\right)^{2} .
\end{aligned}
$$

Here $\theta \in[0, \pi]$ and $\varphi \in \mathbb{R} / 2 \pi \mathbb{Z}$ are the spherical coordinates on $\mathbb{S}^{2}$ and $r, t$ take values in $\mathbb{R} ; M_{0}$ is the mass of the black hole, $\Lambda$ is the cosmological constant, and $a$ is the angular momentum;

$$
\begin{aligned}
\Delta_{r} & =\left(r^{2}+a^{2}\right)\left(1-\frac{\Lambda r^{2}}{3}\right)-2 M_{0} r, \quad \Delta_{\theta}=1+\alpha \cos ^{2} \theta, \\
\rho^{2} & =r^{2}+a^{2} \cos ^{2} \theta, \quad \alpha=\frac{\Lambda a^{2}}{3} .
\end{aligned}
$$

The metric in the $(t, r, \theta, \varphi)$ coordinates is defined for $\Delta_{r}>0$; we assume that this happens on an open interval $r \in\left(r_{-}, r_{+}\right)$, where $r_{ \pm}$are two of the roots of the fourth order polynomial equation $\Delta_{r}(r)=0$. The metric becomes singular at $r=r_{ \pm}$; however, this apparent singularity goes away if we consider the following version of the Kerr-star coordinates (see [18, Section 5.1] and [47]):

$$
t^{*}=t-F_{t}(r), \varphi^{*}=\varphi-F_{\varphi}(r),
$$

with the functions $F_{t}, F_{\varphi}$ blowing up like $c_{ \pm} \log \left|r-r_{ \pm}\right|$as $r$ approaches $r_{ \pm}$. One can choose $F_{t}, F_{\varphi}$ so that the metric continues smoothly across the surfaces $\left\{r=r_{ \pm}\right\}$, called event horizons, to

$$
M_{\delta}=\mathbb{R}_{t} \times X_{\delta}, X_{\delta}=\left(r_{-}-\delta, r_{+}+\delta\right) \times \mathbb{S}^{2},
$$

with $\delta>0$ is a small constant. Moreover, the surfaces $\left\{t^{*}=\right.$ const $\}$ are spacelike, while the surfaces $\{r=$ const $\}$ are timelike for $r \in\left(r_{-}, r_{+}\right)$, spacelike for $r \notin\left[r_{-}, r_{+}\right]$, and null for $r \in\left\{r_{-}, r_{+}\right\}$. See [26, Section 1], [27, Section 1.1], or [50, Section 6.4] for more information on how to construct $F_{t}, F_{\varphi}$ with these properties. 
Let $\square_{g}$ be the d'Alembert-Beltrami operator of the Kerr-de Sitter metric. Take $f \in H^{s-1}\left(M_{\delta}\right)$ for some $s \geq 1$, and furthermore assume that $f$ is supported in $\left\{0 \leq t^{*} \leq 1\right\}$. Then, since the boundary of $M_{\delta}$ is spacelike and every positive time oriented vector at $\partial M_{\delta}$ points outside of $M_{\delta}$, by the theory of hyperbolic equations (see for example [18, Proposition 3.1.1] or [48, Sections 2.8 and 7.7]) there exists unique solution $u \in H_{\mathrm{loc}}^{s}\left(M_{\delta}\right)$ to the problem

$$
\square_{g} u=f, \operatorname{supp} u \subset\left\{t^{*} \geq 0\right\} .
$$

We will henceforth consider the problem (1.2); the Cauchy problem (0.5) can be reduced to (1.2) as follows. Assume that $u$ solves (0.5) with some $f_{0} \in H^{s}\left(X_{\delta}\right)$, $f_{1} \in H^{s-1}\left(X_{\delta}\right)$. Take a function $\chi \in C^{\infty}(\mathbb{R})$ such that supp $\chi \subset\left\{t^{*}>0\right\}$ and $\operatorname{supp}(1-\chi) \subset\left\{t^{*}<1\right\}$; then, $\chi\left(t^{*}\right) u$ solves (1.2) with $f=\left[\square_{g}, \chi\right] u$ supported in $\left\{0 \leq t^{*} \leq 1\right\}$ and the $H^{s-1}$ norm of $f$ is controlled by $\left\|f_{0}\right\|_{H^{s}}+\left\|f_{1}\right\|_{H^{s-1}}$.

Since the metric is stationary, there exists a constant $C_{e}$ such that every solution $u$ to (1.2) grows slower than $e^{\left(C_{e}-1\right) t^{*}}$; see [26, Proposition 1.1]. Therefore, the Fourier-Laplace transform

$$
\hat{u}(\omega)=\int e^{i \omega t^{*}} u\left(t^{*}\right) \mathrm{d} t^{*}
$$

is well defined and holomorphic in $\left\{\operatorname{Im} \omega \geq C_{e}\right\}$. Here both $u\left(t^{*}\right)$ and $u(\omega)$ are functions on $X_{\delta}$. Moreover, if $\hat{f}(\omega)$ is the Fourier-Laplace transform of $f$, then

$$
P_{g}(\omega) \hat{u}(\omega)=\rho^{2} \hat{f}(\omega), \quad \operatorname{Im} \omega \geq C_{e},
$$

where $P_{g}(\omega)$ is the stationary d'Alembert-Beltrami operator, obtained by replacing $D_{t^{*}}$ with $-\omega$ in $\rho^{2} \square_{g}$. (The $\rho^{2}$ factor will prove useful in the next subsection). Finally, since $f$ is supported in $\left\{0 \leq t^{*} \leq 1\right\}$, the function $\hat{f}(\omega)$ is holomorphic in the entire $\mathbb{C}$, and

$$
\left\|\langle\omega\rangle^{s-1} \hat{f}(\omega)\right\|_{H_{\langle\omega\rangle}^{s-1}\left(X_{\delta}\right)} \leq C\|f\|_{H^{s-1}\left(M_{\delta}\right)}
$$

for $\operatorname{Im} \omega$ bounded by a fixed constant. Here $H_{h}^{s-1}, h>0$, is the semiclassical Sobolev space, consisting of the same functions as $H^{s-1}$, but with norm $\left\|\langle h D\rangle^{s-1} f\right\|_{L^{2}}$ instead of $\left\|\langle D\rangle^{s-1} f\right\|_{L^{2}}$.

If $P_{g}(\omega)$ was, say, an elliptic operator, then the Eq. (1.3) would have many solutions; however, because of the degeneracies occurring at the event horizons, the requirement that $\hat{u} \in H^{s}$ acts as a boundary condition. This situation was examined in detail in [50]; the following proposition follows from [50, Theorem 1.2 and Lemma 3.1] (see [26, Proposition 1.2] for the cutoff version):

Proposition 1.1. Fix $\nu_{0}>0$. Then, for s large enough depending on $\nu_{0}$, there exists a family of operators (called the scattering resolvent)

$$
R(\omega): H^{s-1}\left(X_{\delta}\right) \rightarrow H^{s}\left(X_{\delta}\right), \quad \operatorname{Im} \omega \geq-\nu_{0},
$$

meromorphic with poles of finite rank and such that for $u$ solving (1.2), we have

$$
\hat{u}(\omega)=R(\omega) \hat{f}(\omega), \quad \operatorname{Im} \omega \geq C_{e} .
$$


Note that even though we originally defined the left-hand side of (1.5) for $\operatorname{Im} \omega \geq C_{e}$, the right-hand side of this equation makes sense in a wider region $\operatorname{Im} \omega \geq-\nu_{0}$, and in fact in the entire complex plane if $f$ is smooth. The idea now is to use Fourier inversion formula

$$
u\left(t^{*}\right)=\frac{1}{2 \pi} \int_{\operatorname{Im} \omega=C_{e}} e^{-i \omega t^{*}} R(\omega) \hat{f}(\omega) \mathrm{d} \omega
$$

and deform the contour of integration to $\left\{\operatorname{Im} \omega=-\nu_{0}\right\}$ to get exponential decay via the $e^{-i \omega t^{*}}$ factor. We pick up residues from the poles of $R(\omega)$ when deforming the contour; therefore, one defines quasi-normal modes as the poles of $R(\omega)$.

Our ability to deform the contour and estimate the resulting integral depends on having polynomial resolvent estimates. To formulate these, let us give the technical.

Definition 1.1. Let $h>0$ be a parameter and $\mathcal{R}(\omega ; h): \mathcal{H}_{1} \rightarrow \mathcal{H}_{2}, \omega \in \mathcal{U}(h) \subset$ $\mathbb{C}$, be a meromorphic family of operators, with $\mathcal{H}_{j}$ Hilbert spaces. Let also $\Omega(h) \subset \mathcal{U}(h)$ be open and $\mathcal{Z}(h) \subset \mathbb{C}$ be a finite subset; we allow elements of $\mathcal{Z}(h)$ to have multiplicities. We say that the poles of $\mathcal{R}$ in $\Omega(h)$ are simple with a polynomial resolvent estimate and given modulo $O\left(h^{\infty}\right)$ by $\mathcal{Z}(h)$, if for $h$ small enough, there exist maps $\mathcal{Q}$ and $\Pi$ from $\mathcal{Z}(h)$ to $\mathbb{C}$ and the algebra of bounded operators $\mathcal{H}_{1} \rightarrow \mathcal{H}_{2}$, respectively, such that:

- for each $\widehat{\omega}^{\prime} \in \mathcal{Z}(h), \widehat{\omega}=\mathcal{Q}\left(\widehat{\omega}^{\prime}\right)$ is a pole of $\mathcal{R},\left|\widehat{\omega}-\widehat{\omega}^{\prime}\right|=O\left(h^{\infty}\right)$, and $\Pi\left(\widehat{\omega}^{\prime}\right)$ is a rank one operator;

- there exists a constant $N$ such that $\left\|\Pi\left(\widehat{\omega}^{\prime}\right)\right\|_{\mathcal{H}_{1} \rightarrow \mathcal{H}_{2}}=O\left(h^{-N}\right)$ for each $\widehat{\omega}^{\prime} \in \mathcal{Z}(h)$ and, moreover,

$$
\mathcal{R}(\omega ; h)=\sum_{\widehat{\omega}^{\prime} \in \mathcal{Z}(h)} \frac{\Pi\left(\widehat{\omega}^{\prime}\right)}{\omega-\mathcal{Q}\left(\widehat{\omega}^{\prime}\right)}+O_{\mathcal{H}_{1} \rightarrow \mathcal{H}_{2}}\left(h^{-N}\right), \quad \omega \in \Omega(h) .
$$

In particular, every pole of $\mathcal{R}$ in $\Omega(h)$ lies in the image of $\mathcal{Q}$.

The quantization condition and resolvent estimate that we need to prove Theorems 1 and 2 are contained in

Proposition 1.2. Fix $\nu_{0}>0$ and let $h>0$ be a parameter. Then, for a small enough (independently of $\nu_{0}$ ), the poles of $R(\omega)$ in the region

$$
|\operatorname{Im} \omega|<\nu_{0}, h^{-1}<|\operatorname{Re} \omega|<2 h^{-1},
$$

are simple with a polynomial resolvent estimate and given modulo $O\left(h^{\infty}\right)$ by

$$
\begin{gathered}
\omega=h^{-1} \mathcal{F}^{\omega}(m, h l, h k ; h), m, l, k \in \mathbb{Z}, \\
0 \leq m \leq C_{m}, C_{l}^{-1} \leq h l \leq C_{l},|k| \leq l .
\end{gathered}
$$

Here $C_{m}$ and $C_{l}$ are some constants and $\mathcal{F}^{\omega}(m, \tilde{l}, \tilde{k} ; h)$ is a classical symbol:

$$
\mathcal{F}^{\omega}(m, \tilde{l}, \tilde{k} ; h) \sim \sum_{j \geq 0} h^{j} \mathcal{F}_{j}^{\omega}(m, \tilde{l}, \tilde{k}) .
$$


The principal symbol $\mathcal{F}_{0}^{\omega}$ is real-valued and independent of $m$; moreover,

$$
\begin{aligned}
\mathcal{F}^{\omega}(m, \tilde{l}, \tilde{k} ; h)= & \frac{\sqrt{1-9 \Lambda M_{0}^{2}}}{3 \sqrt{3} M_{0}}(\tilde{l}+h / 2-i h(m+1 / 2))+O\left(h^{2}\right) \quad \text { for } a=0, \\
& \left(\partial_{\tilde{k}} \mathcal{F}_{0}^{\omega}\right)(m, \pm \tilde{k}, \tilde{k})=\frac{\left(2+9 \Lambda M_{0}^{2}\right) a}{27 M_{0}^{2}}+O\left(a^{2}\right) .
\end{aligned}
$$

Finally, if we consider $R(\omega)$ as a family of operators between the semiclassical Sobolev spaces $H_{h}^{s-1} \rightarrow H_{h}^{s}$, then the constant $N$ in Definition 1.1 is independent of $s$.

Theorem 1 follows from here almost immediately. Indeed, since $R(\omega)$ is independent of $h$, each $\mathcal{F}_{j}^{\omega}$ is homogeneous in $(\tilde{l}, \tilde{k})$ variables of degree $1-j$; we can then extend this function homogeneously to the cone $|\tilde{k}| \leq \tilde{l}$ and define the (nonsemiclassical) symbol

$$
\mathcal{F}(m, l, k) \sim \sum_{j \geq 0} \mathcal{F}_{j}^{\omega}(m, l, k) .
$$

Note that $\mathcal{F}(m, l, k)=h^{-1} \mathcal{F}^{\omega}(m, h l, h k ; h)+O\left(h^{\infty}\right)$ whenever $C_{l}^{-1} \leq h l \leq C_{l}$. We can then cover the region $(0.1)$ for large $C_{\omega}$ with the regions (1.7) for a sequence of small values of $h$ to see that QNMs in (0.1) are given by $(0.2)$ modulo $O\left(|\omega|^{-\infty}\right)$.

Now, we prove Theorem 2. Let $u$ be a solution to (1.2), with $f \in H^{s-1}$ and $s$ large enough. We claim that one can deform the contour in (1.6) to get $u\left(t^{*}\right)=i \sum_{\operatorname{Im} \widehat{\omega}>-\nu_{0}} \operatorname{Res}_{\omega=\widehat{\omega}}\left[e^{-i \omega t^{*}} R(\omega) \hat{f}(\omega)\right]+\frac{1}{2 \pi} \int_{\operatorname{Im} \omega=-\nu_{0}} e^{-i \omega t^{*}} R(\omega) \hat{f}(\omega) \mathrm{d} \omega$.

The series in (1.9) is over QNMs $\widehat{\omega}$; all but a finite number of them in the region $\left\{\operatorname{Im} \omega>-\nu_{0}\right\}$ are equal to $\mathcal{Q}\left(\widehat{\omega}^{\prime}\right)$ for some $\widehat{\omega}^{\prime}$ given by $(0.2)$ and the residue in this case is $e^{-i \widehat{\omega} t^{*}} \Pi\left(\widehat{\omega}^{\prime}\right) \hat{f}(\widehat{\omega})$. Here $\mathcal{Q}$ and $\Pi$ are taken from Definition 1.1. Now, by (1.4), we have

$$
\begin{aligned}
\left\|\Pi\left(\widehat{\omega}^{\prime}\right) \hat{f}(\widehat{\omega})\right\|_{H^{1}} & \leq C\langle\widehat{\omega}\rangle^{N-s}\|f\|_{H^{s-1}} ; \\
\|R(\omega) \hat{f}(\omega)\|_{H^{1}} & \leq C\langle\omega\rangle^{N-s}\|f\|_{H^{s-1}}, \quad \operatorname{Im} \omega=-\nu_{0},
\end{aligned}
$$

for some constant $N$ independent of $s$; therefore, for $s$ large enough, the series in (1.9) converges in $H^{1}$ and the $H^{1}$ norm of the integral in (1.9) can be estimated by $C e^{-\nu_{0} t^{*}}\|f\|_{H^{s-1}}$, thus proving Theorem 2 .

To prove (1.9), take small $h>0$. There are $O\left(h^{-2}\right)$ QNMs in the region (1.7); therefore, by pigeonhole principle we can find $\omega_{0}(h) \in\left[h^{-1}, 2 h^{-1}\right]$ such that there are no QNMs $h^{2}$-close to the segments

$$
\gamma_{ \pm}(h)=\left\{\operatorname{Re} \omega= \pm \omega_{0}(h),-\nu_{0} \leq \operatorname{Im} \omega \leq C_{e}\right\} .
$$

Then, $\|R(\omega) \hat{f}(\omega)\|_{H^{1}}=O\left(h^{s-N-4}\right)$ on $\gamma_{ \pm}(h)$; we can now apply the residue theorem to the rectangle formed from $\gamma_{ \pm}(h)$ and segments of the lines $\left\{\operatorname{Im} \omega=C_{e}\right\},\left\{\operatorname{Im} \omega=-\nu_{0}\right\}$, and then let $h \rightarrow 0$. 


\subsection{Separation of Variables}

First of all, using [50, (A.2), (A.3)], we reduce Proposition 1.2 to the following ${ }^{4}$

Proposition 1.3. Take $\delta>0$ and put

$$
K_{\delta}=\left(r_{-}+\delta, r_{+}-\delta\right) \times \mathbb{S}^{2}, R_{g}(\omega)=1_{K_{\delta}} R(\omega) 1_{K_{\delta}}: L^{2}\left(K_{\delta}\right) \rightarrow H^{2}\left(K_{\delta}\right) .
$$

Then, for a small enough $h^{5}$ and fixed $\nu_{0}$, the poles of $R_{g}(\omega)$ in the region (1.7) are simple with a polynomial resolvent estimate $L^{2} \rightarrow L^{2}$ and given modulo $O\left(h^{\infty}\right)$ by (1.8).

Furthermore, by [50, Proposition A.1] the family of operators $R_{g}(\omega)$ coincides with the one constructed in [26, Theorem 2], if the functions $F_{t}, F_{\varphi}$ in (1.1) are chosen so that $(t, \varphi)=\left(t^{*}, \varphi^{*}\right)$ in $\mathbb{R}_{t} \times K_{\delta}$. We now review how the construction of $R_{g}(\omega)$ in [26] works and reduce Proposition 1.3 to two separate spectral problems in the radial and the angular variables. For the convenience of reader, we include the simpler separation of variables procedure for the case $a=0$ at the end of this section.

First of all, the operator $P_{g}(\omega)$ is invariant under the rotation $\varphi \mapsto \varphi+$ $s$; therefore, the spaces $\mathcal{D}_{k}^{\prime}=\operatorname{Ker}\left(D_{\varphi}-k\right)$ of functions of angular momentum $k \in \mathbb{Z}$ are invariant under both $P_{g}(\omega)$ and $R_{g}(\omega)$. In [26], we construct $R_{g}(\omega)$ by piecing together the restrictions $R_{g}(\omega, k)=\left.R_{g}(\omega)\right|_{\mathcal{D}_{k}^{\prime}}$ for all $k$. Then, Proposition 1.3 follows from

Proposition 1.4. Under the assumptions of Proposition 1.3, there exists a constant $C_{k}$ such that for each $k \in \mathbb{Z}$,

1. if $h|k|>C_{k}$, then $R_{g}(\omega, k)$ has no poles in the region (1.7) and its $L^{2} \rightarrow L^{2}$ norm is $O\left(|k|^{-2}\right)$; (This is a reformulation of [26, Proposition 3.3]).

2. if $h|k| \leq C_{k}$, then the poles of $R_{g}(\omega, k)$ in the region (1.7) are simple with a polynomial resolvent estimate $L^{2} \rightarrow L^{2}$ and given modulo $O\left(h^{\infty}\right)$ by (1.8), with this particular value of $k$.

Now, we recall from [26, Section 1] that the restriction of $P_{g}(\omega)$ to $\mathcal{D}_{k}^{\prime}$ has the form ${ }^{6} P_{r}(\omega, k)+\left.P_{\theta}(\omega)\right|_{\mathcal{D}_{k}^{\prime}}$, where

$$
\begin{aligned}
P_{r}(\omega, k) & =D_{r}\left(\Delta_{r} D_{r}\right)-\frac{(1+\alpha)^{2}}{\Delta_{r}}\left(\left(r^{2}+a^{2}\right) \omega-a k\right)^{2}, \\
P_{\theta}(\omega) & =\frac{1}{\sin \theta} D_{\theta}\left(\Delta_{\theta} \sin \theta D_{\theta}\right)+\frac{(1+\alpha)^{2}}{\Delta_{\theta} \sin ^{2} \theta}\left(a \omega \sin ^{2} \theta-D_{\varphi}\right)^{2}
\end{aligned}
$$

are differential operators in $r$ and $(\theta, \varphi)$, respectively. Then, $R_{g}(\omega, k)$ is constructed in [26, Proof of Theorem 1] using a certain contour integral [26, (2.1)]

\footnotetext{
${ }^{4}$ One could also try to apply the results of [20] here, but we use the slightly simpler construction of [50, Appendix], exploiting the fact that we have information on the exact cutoff resolvent.

5 The smallness of $a$ is implied in all following statements.

6 The operator $P_{g}(\omega)$ of [26] differs from our operator by the conjugation done in [50, Appendix]; however, the two coincide in $K_{\delta}$.
} 
and the radial and angular resolvents

$$
\begin{aligned}
R_{r}(\omega, \lambda, k) & : L_{\mathrm{comp}}^{2}\left(r_{-}, r_{+}\right) \rightarrow H_{\mathrm{loc}}^{2}\left(r_{-}, r_{+}\right), \\
R_{\theta}(\omega, \lambda) & : L^{2}\left(\mathbb{S}^{2}\right) \rightarrow H^{2}\left(\mathbb{S}^{2}\right), \lambda \in \mathbb{C} ;
\end{aligned}
$$

$R_{r}$ is a certain right inverse to $P_{r}(\omega, k)+\lambda$, while $R_{\theta}$ is the inverse to $P_{\theta}(\omega)-\lambda$; we write $R_{\theta}(\omega, \lambda, k)=\left.R_{\theta}(\omega, \lambda)\right|_{\mathcal{D}_{k}^{\prime}}$. Recall that both $R_{r}$ and $R_{\theta}$ are meromorphic families of operators, as defined in [26, Definition 2.1]; in particular, for a fixed value of $\omega$, these families are meromorphic in $\lambda$ with poles of finite rank. By definition of $R_{g}(\omega, k)$, a number $\omega \in \mathbb{C}$ is a pole of this operator if and only if there exists $\lambda \in \mathbb{C}$ such that $(\omega, \lambda, k)$ is a pole of both $R_{r}$ and $R_{\theta}$.

Now, for small $h>0$ we put

$$
\tilde{\omega}=h \operatorname{Re} \omega, \quad \tilde{\nu}=\operatorname{Im} \omega, \quad \tilde{\lambda}=h^{2} \operatorname{Re} \lambda, \quad \tilde{\mu}=h \operatorname{Im} \lambda, \quad \tilde{k}=h k ;
$$

the assumptions of Proposition 1.4(2) imply that $1 \leq \tilde{\omega} \leq 2,|\tilde{\nu}| \leq \nu_{0}$, and $|\tilde{k}| \leq C_{k}$. Moreover, [26, Proposition 3.4] suggests that under these assumptions, all values of $\lambda$ for which $(\omega, \lambda, k)$ is a pole of both $R_{r}$ and $R_{\theta}$ have to satisfy $|\tilde{\lambda}|,|\tilde{\mu}| \leq C_{\lambda}$, for some constant $C_{\lambda}$.

We are now ready to state the quantization conditions and resolvent estimates for $R_{r}$ and $R_{\theta}$; the former is proved in Sect. 4 and the latter, in Sect. 3.

Proposition 1.5 (Radial lemma). Let $C_{\lambda}$ be a fixed constant and put $K_{r}=$ $\left(r_{-}+\delta, r_{+}-\delta\right)$. Then, the poles of $1_{K_{r}} R_{r}(\omega, \lambda, k) 1_{K_{r}}$ as a function of $\lambda$ in the region

$$
1<\tilde{\omega}<2,|\tilde{\nu}|<\nu_{0},|\tilde{k}|<C_{k},|\tilde{\lambda}|,|\tilde{\mu}|<C_{\lambda},
$$

are simple with polynomial resolvent estimate $L^{2} \rightarrow L^{2}$ (in the sense of Definition 1.1) and given modulo $O\left(h^{\infty}\right)$ by

$$
\tilde{\lambda}+i h \tilde{\mu}=\mathcal{F}^{r}(m, \tilde{\omega}, \tilde{\nu}, \tilde{k} ; h), m \in \mathbb{Z}, 0 \leq m \leq C_{m},
$$

for some constant $C_{m}$. The principal part $\mathcal{F}_{0}^{r}$ of the classical symbol $\mathcal{F}^{r}$ is real-valued, independent of $m$ and $\tilde{\nu}$, and

$$
\begin{gathered}
\mathcal{F}^{r}=\left[i h(m+1 / 2)+\frac{3 \sqrt{3} M_{0}}{\sqrt{1-9 \Lambda M_{0}^{2}}}(\tilde{\omega}+i h \tilde{\nu})\right]^{2}+O\left(h^{2}\right) \quad \text { for } a=0, \\
\mathcal{F}_{0}^{r}(\tilde{\omega}, \tilde{k})=\frac{27 M_{0}^{2}}{1-9 \Lambda M_{0}^{2}} \tilde{\omega}^{2}-\frac{6 a \tilde{k} \tilde{\omega}}{1-9 \Lambda M_{0}^{2}}+O\left(a^{2}\right) .
\end{gathered}
$$

In particular, for $\omega, k$ satisfying (1.13), every pole $\lambda$ satisfies $\tilde{\lambda}>\varepsilon$ for some constant $\varepsilon>0$.

Proposition 1.6 (Angular lemma). Let $C_{\theta}$ be a fixed constant. Then, the poles of $R_{\theta}(\omega, \lambda, k)$ as a function of $\lambda$ in the region

$$
1<\tilde{\omega}<2, \quad|\tilde{\nu}|<\nu_{0}, \quad|\tilde{k}|<C_{k}, \quad C_{\theta}^{-1}<\tilde{\lambda}<C_{\theta}, \quad|\tilde{\mu}|<C_{\theta},
$$

are simple with polynomial resolvent estimate $L^{2} \rightarrow L^{2}$ and given modulo $O\left(h^{\infty}\right)$ by

$$
\tilde{\lambda}+i h \tilde{\mu}=\mathcal{F}^{\theta}(h l, \tilde{\omega}, \tilde{\nu}, \tilde{k} ; h), \quad l \in \mathbb{Z}, \quad \max \left(|\tilde{k}|, C_{l}^{-1}\right) \leq h l \leq C_{l},
$$




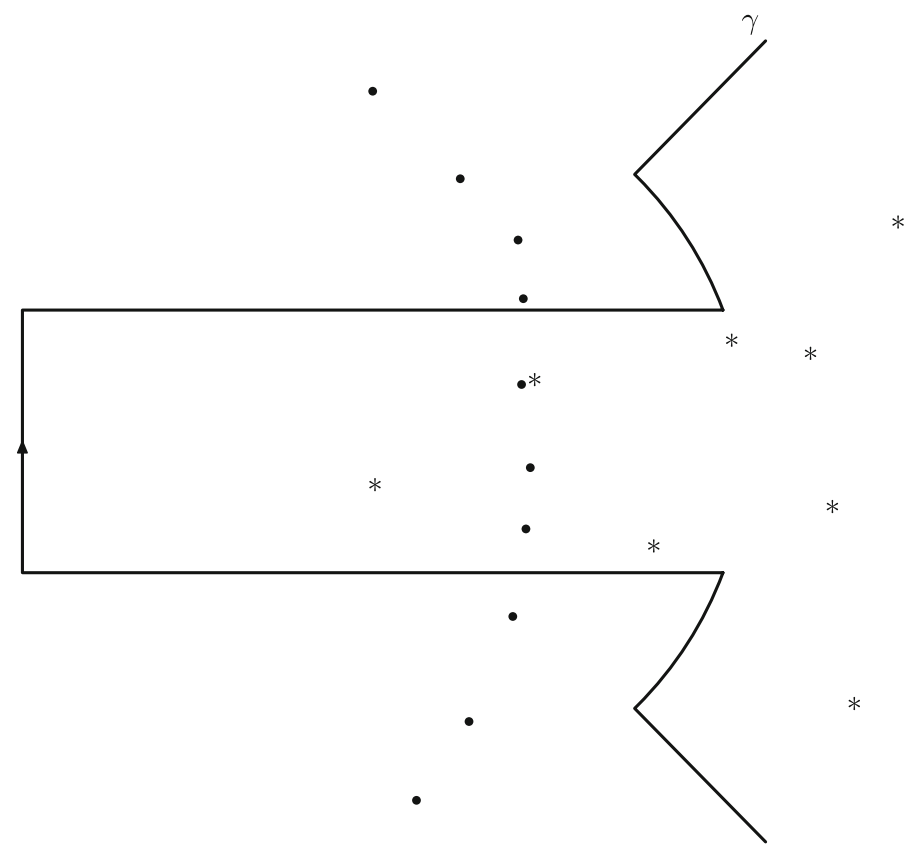

Figure 1. The contour $\gamma$ and interaction between radial poles (denoted by dots) and angular poles (denoted by asterisks)

for some constant $C_{l}$. The principal part $\mathcal{F}_{0}^{\theta}$ of the classical symbol $\mathcal{F}^{\theta}$ is real-valued, independent of $\tilde{\nu}$, and

$$
\mathcal{F}^{\theta}=\tilde{l}(\tilde{l}+h)+O\left(h^{\infty}\right) \quad \text { for } a=0 .
$$

Moreover, $\mathcal{F}_{0}^{\theta}( \pm \tilde{k}, \tilde{\omega}, \tilde{k})=(1+\alpha)^{2}(\tilde{k}-a \tilde{\omega})^{2}, \partial_{\tilde{l}} \mathcal{F}_{0}^{\theta}( \pm \tilde{k}, \tilde{\omega}, \tilde{k})= \pm 2 \tilde{k}+O\left(a^{2}\right)$, and consequently, $\partial_{\tilde{k}} \mathcal{F}_{0}^{\theta}( \pm \tilde{k}, \tilde{\omega}, \tilde{k})=-2 a \tilde{\omega}+O\left(a^{2}\right)$.

Combining Propositions 1.5 and 1.6 with the results of [26], we get

Proof of Proposition 1.4. We let $\mathcal{F}^{\omega}(m, \tilde{l}, \tilde{k} ; h)$ be the solution $\tilde{\omega}+i h \tilde{\nu}$ to the equation

$$
\mathcal{F}^{r}(m, \tilde{\omega}, \tilde{\nu}, \tilde{k} ; h)=\mathcal{F}^{\theta}(\tilde{l}, \tilde{\omega}, \tilde{\nu}, \tilde{k} ; h) .
$$

We can see that this equation has unique solution by writing $\mathcal{F}^{r}-\mathcal{F}^{\theta}=$ $\mathcal{F}^{\prime}+i h \mathcal{F}^{\prime \prime}$ and examining the principal parts of the real-valued symbols $\mathcal{F}^{\prime}, \mathcal{F}^{\prime \prime}$ for $a=0$.

The idea now is to construct an admissible contour in the sense of [26, Definition 2.3]; e.g., a contour that separates the sets of poles (in the variable $\lambda$ ) of $R_{r}$ and $R_{\theta}$ from each other; then, [26, (2.1)] provides a formula for $R_{g}(\omega, k)$, which can be used to get a resolvent estimate.

We will use the method of proof of [26, Proposition 3.4]. Take the contour $\gamma$ introduced there (Fig. 1), for $l_{2}=C_{\lambda} h^{-1}, l_{1}=L=C_{\lambda} h^{-2}$, and $C_{\lambda}$ some 
large constant. Then, we know that all angular poles are to the right of $\gamma$ (in $\left.\Gamma_{2}\right)$. Moreover, the only radial poles to the right of $\gamma$ lie in the domain $\{|\operatorname{Im} \lambda| \leq$ $\left.l_{2},|\lambda| \leq L\right\}$ and they are contained in the set $\left\{\lambda_{0}^{r}, \ldots, \lambda_{C_{m}}^{r}\right\}$ for some constant $C_{m}$, where $\lambda_{m}^{r}(\omega, k)$ is the radial pole corresponding to $h^{-2} \mathcal{F}^{r}(m, \tilde{\omega}, \tilde{\nu}, \tilde{k} ; h)$. In particular, those radial poles are contained in

$$
U_{\lambda}=\left\{C_{\theta}^{-1}<\tilde{\lambda}<C_{\theta},|\tilde{\mu}| \leq C_{\theta}\right\}
$$

for some constant $C_{\theta}$.

Assume that $\omega$ is not a pole of $R_{g}(\omega, k)$; then, we can consider the admissible contour composed of $\gamma$ and the circles $\gamma_{m}, 0 \leq m \leq C_{m}$, enclosing $\lambda_{m}^{r}(\omega, k)$, but none of the other poles of $R_{r}$ or $R_{\theta}$. Using the meromorphic decomposition of $R_{r}$ at $\lambda_{m}^{r}$ and letting its principal part be $\Pi_{m}^{r} /\left(\lambda-\lambda_{m}^{r}\right)$, we get

$$
\begin{aligned}
R_{g}(\omega, k)= & \sum_{m} \Pi_{m}^{r}(\omega, k) \otimes R_{\theta}\left(\omega, \lambda_{m}^{r}(\omega, k), k\right) \\
& +\frac{1}{2 \pi i} \int_{\gamma} R_{r}(\omega, \lambda, k) \otimes R_{\theta}(\omega, \lambda, k) d \lambda .
\end{aligned}
$$

Here we only include the poles $\lambda_{m}^{r}$ lying to the right of $\gamma$; one might need to change $l_{1}$ in the definition of $\gamma$ a little bit in case some $\lambda_{m}^{r}$ comes close to $\gamma$. The integral in (1.18) is holomorphic and bounded polynomially in $h$, by the bounds for $R_{r}$ given by Proposition 1.5, together with the estimates in the proof of [26, Proposition 3.4].

Now, the poles of $R_{\theta}$ in $U_{\lambda}$ are given by (1.16); let $\lambda_{l}^{\theta}(\omega, k)$ be the pole corresponding to $h^{-2} \mathcal{F}^{\theta}(h l, \tilde{\omega}, \tilde{\nu}, \tilde{k} ; h)$ and $\Pi_{l}^{\theta} /\left(\lambda-\lambda_{l}^{\theta}\right)$ be the principal part of the corresponding meromorphic decomposition. Then, the resolvent estimates on $R_{\theta}$ given by Proposition 1.6 together with (1.18) imply

$$
R_{g}(\omega, k)=\operatorname{Hol}(\omega)+\sum_{m, l} \frac{\Pi_{m}^{r}(\omega, k) \otimes \Pi_{l}^{\theta}(\omega, k)}{\lambda_{m}^{r}(\omega, k)-\lambda_{l}^{\theta}(\omega, k)} .
$$

Here $\operatorname{Hol}(\omega)$ is a family of operators holomorphic in $\omega$ and bounded polynomially in $h$. Moreover,

$$
\lambda_{m}^{r}(\omega, k)-\lambda_{l}^{\theta}(\omega, k)=h^{-2}\left(\mathcal{F}^{r}(m, \tilde{\omega}, \tilde{\nu}, \tilde{k} ; h)-\mathcal{F}^{\theta}(h l, \tilde{\omega}, \tilde{\nu}, \tilde{k} ; h)\right)+O\left(h^{\infty}\right) ;
$$

therefore, the equation $\lambda_{m}^{r}(\omega, k)-\lambda_{l}^{\theta}(\omega, k)=0$ is an $O\left(h^{\infty}\right)$ perturbation of (1.17) and it has a unique solution $\omega_{m, l}(k)$, which is $O\left(h^{\infty}\right)$ close to $h^{-1} \mathcal{F}^{\omega}(m, h l, \tilde{k} ; h)$. Finally, in the region $(1.7)$ we can write by $(1.19)$

$$
R_{g}(\omega, k)=\operatorname{Hol}(\omega)+\sum_{m, l} \frac{\Pi_{m, l}(k)}{\omega-\omega_{m, l}(k)},
$$

with $\operatorname{Hol}(\omega)$ as above and $\Pi_{m, l}(k)$ being the product of a coefficient polynomially bounded in $h$ with $\left(\Pi_{m}^{r} \otimes \Pi_{l}^{\theta}\right)\left(\omega_{m, l}(k), k\right)$; this finishes the proof. 
Finally, let us present the simplified separation of variables for the case $a=0$, namely the Schwarzschild-de Sitter metric:

$$
\begin{gathered}
g=\frac{\Delta_{r}}{r^{2}} d t^{2}-\frac{r^{2}}{\Delta_{r}} d r^{2}-r^{2}\left(d \theta^{2}+\sin ^{2} \theta d \varphi^{2}\right), \\
\Delta_{r}=r^{2}\left(1-\frac{\Lambda r^{2}}{3}\right)-2 M_{0} r .
\end{gathered}
$$

Note that $d \theta^{2}+\sin ^{2} \theta d \varphi^{2}$ is just the round metric on the unit sphere. The metric decouples without the need to take Fourier series in $\varphi$; the stationary d'Alembert-Beltrami operator has the form $P_{g}(\omega)=P_{r}(\omega)+P_{\theta}$, where

$$
\begin{gathered}
P_{r}(\omega)=D_{r}\left(\Delta_{r} D_{r}\right)-\frac{r^{4}}{\Delta_{r}} \omega^{2}, \\
P_{\theta}=\frac{1}{\sin \theta} D_{\theta}\left(\sin \theta D_{\theta}\right)+\frac{D_{\varphi}^{2}}{\sin ^{2} \theta} .
\end{gathered}
$$

Here $P_{\theta}$ is the Laplace-Beltrami operator on the round sphere; it is self-adjoint (thus, no need for the contour integral construction of [26, Section 2]) and is known to have eigenvalues $l(l+1)$, where $l \geq 0$. Each such eigenvalue has multiplicity $2 l+1$, corresponding to the values $-l, \ldots, l$ of the $\varphi$-angular momentum $k$. The angular Lemma 1.6 follows immediately. (We nevertheless give a more microlocal explanation in this case at the end of Sect. 3.1.) One can now decompose $L^{2}$ into an orthogonal sum of the eigenspaces of $P_{\theta}$; on the space $V_{\lambda}$ corresponding to the eigenvalue $\lambda=l(l+1)$, we have

$$
\left.P_{g}(\omega)\right|_{V_{\lambda}}=P_{r}(\omega)+\lambda .
$$

Therefore, the only problem is to show the radial Lemma 1.5 in this case, which is in fact no simpler than the general case. (Note that we take a different path here than [41] and [10], using only real microlocal analysis near the trapped set, which immediately gives polynomial resolvent bounds).

\section{Preliminaries}

\subsection{Pseudodifferential Operators and Microlocalization}

First of all, we review the classes of semiclassical pseudodifferential operators on manifolds and introduce notation used for these classes; see [54, Sections 9.3 and 14.2] or [21] for more information.

For $k \in \mathbb{R}$, we consider the symbol class $S^{k}\left(\mathbb{R}^{n}\right)$ consisting of functions $a(x, \xi ; h)$ smooth in $(x, \xi) \in \mathbb{R}^{2 n}$ and satisfying the following growth conditions: for each compact set $K \subset \mathbb{R}^{n}$ and each pair of multi-indices $\alpha, \beta$, there exists a constant $C_{\alpha \beta K}$ such that

$$
\left|\partial_{x}^{\alpha} \partial_{\xi}^{\beta} a(x, \xi ; h)\right| \leq C_{\alpha \beta K}\langle\xi\rangle^{k-|\beta|}, \quad x \in K, \xi \in \mathbb{R}^{n}, h>0 .
$$

If we treat $\mathbb{R}^{2 n}$ as the cotangent bundle to $\mathbb{R}^{n}$, then the class $S^{k}$ is invariant under changes of variables; this makes it possible, given a manifold $M$, to define the class $S^{k}(M)$ of symbols depending on $(x, \xi) \in T^{*} M$. 
If $k \in \mathbb{R}$ and $a_{j}(x, \xi) \in S^{k-j}(M), j=0,1, \ldots$, is a sequence of symbols, then there exists the asymptotic sum

$$
a(x, \xi ; h) \sim \sum_{j \geq 0} h^{j} a_{j}(x, \xi) ;
$$

i.e., a symbol $a(x, \xi ; h) \in S^{k}(M)$ such that for every $J=0,1, \ldots$,

$$
a(x, \xi ; h)-\sum_{0 \leq j<J} h^{j} a_{j}(x, \xi) \in h^{J} S^{k-J} .
$$

The asymptotic sum $a$ is unique modulo the class $h^{\infty} S^{-\infty}$ of symbols all of whose derivatives decay faster than $h^{N}\langle\xi\rangle^{-N}$ for each $N$ on any compact set in $x$. If $a$ is given by an asymptotic sum of the form (2.1), then we call it a classical symbol and write $a \in S_{\mathrm{cl}}^{k}(M)$. We call $a_{0}(x, \xi)$ the principal part of the symbol $a(x, \xi ; h)$.

Let $\Psi^{k}(M)$ be the algebra of (properly supported) semiclassical pseudodifferential operators on $M$ with symbols in $S^{k}(M)$. If $H_{h, \text { loc }}^{m}(M), m \in \mathbb{R}$, consists of functions locally lying in the semiclassical Sobolev space, then every element of $\Psi^{k}(M)$ is continuous $H_{h \text {,loc }}^{m}(M) \rightarrow H_{h, \text { loc }}^{m-k}(M)$ with every operator seminorm being $O(1)$ as $h \rightarrow 0$. Let $\Psi_{\mathrm{cl}}^{k}(M)$ be the algebra of operators with symbols in $S_{\mathrm{cl}}^{k}(M)$ and $\Psi_{\mathrm{cl}}(M)$ be the union of $\Psi_{\mathrm{cl}}^{k}$ for all $k$. Next, let the operator class $h^{\infty} \Psi^{-\infty}(M)$ correspond to the symbol class $h^{\infty} S^{-\infty}(M)$; it can be characterized as follows: $A \in h^{\infty} \Psi^{-\infty}(M)$ if and only if for each $N, A$ is continuous $H_{h, \text { loc }}^{-N}(M) \rightarrow H_{h, \text { loc }}^{N}(M)$, with every operator seminorm being $O\left(h^{N}\right)$. The full symbol of an element of $\Psi^{k}(M)$ cannot be recovered as a function on $T^{*} M$; however, if $A \in \Psi_{\mathrm{cl}}^{k}(M)$, then the principal symbol of $A$ is an invariantly defined function on the cotangent bundle. If $M$ is an open subset of $\mathbb{R}^{n}$, then we can define the full symbol of a pseudodifferential operator modulo $h^{\infty} S^{-\infty}$; we will always use Weyl quantization.

We now introduce microlocalization; see also [54, Section 8.4] and [44, Section 3]. Define $U \subset T^{*} M$ to be conic at infinity, if there exists a conic set $V \subset T^{*} M$ such that the symmetric difference of $U$ and $V$ is bounded when restricted to every compact subset of $M$. For $a \in S^{k}(M)$ and $U \subset T^{*} M$ open and conic at infinity, we say that $a$ is rapidly decaying on $U$, if for every $V \subset U$ closed in $T^{*} M$, conic at infinity, and with compact projection onto $M$, every derivative of a decays on $V$ faster than $h^{N}\langle\xi\rangle^{-N}$ for every $N$. We say that $A \in \Psi^{k}(M)$ vanishes microlocally on $U$ if its full symbol (in any coordinate system) is rapidly decaying on $U$. If $A, B \in \Psi^{k}(M)$, then we say that $A=B$ microlocally on $U$, if $A-B$ vanishes microlocally on $U$.

For $A \in \Psi^{k}(M)$, we define the semiclassical wavefront set $\mathrm{WF}_{h}(A) \subset$ $T^{*} M$ as follows: $(x, \xi) \notin \mathrm{WF}_{h}(A)$ if and only if $A$ vanishes microlocally on some neighborhood of $(x, \xi)$. The set $\mathrm{WF}_{h}(A)$ is closed; however, it need not be conic at infinity. Next, we say that $A$ is compactly microlocalized, if there exists a compact set $K \subset T^{*} M$ such that $A$ vanishes microlocally on $T^{*} M \backslash K$. We denote by $\Psi^{\text {comp }}(M)$ the set of compactly microlocalized operators. Here are some properties of microlocalization: 
- If $A$ vanishes microlocally on $U_{1}$ and $U_{2}$, then it vanishes microlocally on $U_{1} \cup U_{2}$.

- The set of pseudodifferential operators vanishing microlocally on some open and conic at infinity $U \subset T^{*} M$ is a two-sided ideal; so is the set of operators with wavefront set contained in some closed $V \subset T^{*} M$. In particular, $\mathrm{WF}_{h}(A B) \subset \mathrm{WF}_{h}(A) \cap \mathrm{WF}_{h}(B)$.

- $A$ vanishes microlocally on the whole $T^{*} M$ if and only if it lies in $h^{\infty} \Psi^{-\infty}$.

- If $A$ vanishes microlocally on $U$, then $\mathrm{WF}_{h}(A) \cap U=\emptyset$; the converse is true if $U$ is bounded. However, ${ }^{7} A$ does not necessarily vanish microlocally on the complement of $\mathrm{WF}_{h}(A)$; for example, the operator $A=e^{-1 / h}$ lies in $\Psi^{0}$ and has an empty wavefront set, yet it does not lie in $h^{\infty} \Psi^{-\infty}$.

- The set $\Psi^{\text {comp }}$ forms a two-sided ideal and it lies in $\Psi^{-N}$ for every $N$.

- Each $A \in \Psi^{\text {comp }}$ vanishes microlocally on the complement of $\mathrm{WF}_{h}(A)$.

- Let $A \in \Psi_{\mathrm{cl}}^{k}(M)$ and let its symbol in some coordinate system have the form (2.1); introduce

$$
V=\bigcup_{j \geq 0} \operatorname{supp} a_{j} .
$$

Then, $A$ vanishes microlocally on some open set $U$ if and only if $U \cap V=\emptyset$; $A$ is compactly microlocalized if $V$ is bounded, and $\mathrm{WF}_{h}(A)$ is the closure of $V$.

We now consider microlocally defined operators. Let $U \subset T^{*} M$ be open. A local pseudodifferential operator $A$ on $U$ is, by definition, a map

$$
B \mapsto[A \cdot B] \in \Psi^{\mathrm{comp}}(M) / h^{\infty} \Psi^{-\infty}(M), B \in \Psi^{\mathrm{comp}}(M), \mathrm{WF}_{h}(B) \subset U,
$$

such that:

- $\mathrm{WF}_{h}([A \cdot B]) \subset \mathrm{WF}_{h}(B)$.

- If $B_{1}, B_{2} \in \Psi^{\operatorname{comp}}(M)$ and $\mathrm{WF}_{h}\left(B_{j}\right) \subset U$, then $\left[A \cdot\left(B_{1}+B_{2}\right)\right]=[A$. $\left.B_{1}\right]+\left[A \cdot B_{2}\right]$.

- If $C \in \Psi^{k}(M)$, then $[A \cdot B] C=[A \cdot(B C)]$.

We denote by $\Psi^{\text {loc }}(U)$ the set of all local operators on $U$. Note that a local operator is only defined modulo an $h^{\infty} \Psi^{-\infty}$ remainder. If $A \in \Psi^{k}(M)$, then the corresponding local operator $\widetilde{A}$ is given by $[\widetilde{A} \cdot B]=A B \bmod h^{\infty} \Psi^{-\infty}$; we say that $A$ represents $\widetilde{A}$. For $M=\mathbb{R}^{n}$ and $U \subset T^{*} M$, there is a one-to-one correspondence between local operators and their full symbols modulo $h^{\infty}$; the symbols of local operators are functions $a(x, \xi ; h)$ smooth in $(x, \xi) \in U$ all of whose derivatives are uniformly bounded in $h$ on compact subsets of $U$. In fact, for a symbol $a(x, \xi ; h)$, the corresponding local operator is defined by $[A \cdot B]=(a \# b)^{w}\left(x, h D_{x}\right)$, where $b(x, \xi ; h)$ is the full symbol of $B \in \Psi^{\text {comp; }}$ since $b$ is compactly supported inside $U$ modulo $O\left(h^{\infty}\right)$ and $a$ is defined on $U$, we can define the symbol product $a \# b$ uniquely modulo $O\left(h^{\infty}\right)$. In particular, a classical local operator $A \in \Psi_{\mathrm{cl}}^{\text {loc }}(M)$ is uniquely determined by the terms of

\footnotetext{
7 This issue can be avoided if we consider $\mathrm{WF}_{h}(A)$ as a subset of the fiber compactified cotangent bundle $\overline{T^{*} M}$, as in [50, Section 2.1]. Then, an operator is compactly microlocalized if and only if its wavefront set does not intersect the fiber infinity.
} 
the decomposition (2.1) of its full symbol. Note that $U$ is not required to be conic at infinity, and we do not impose any conditions on the growth of $a$ as $\xi \rightarrow \infty$.

Local operators form a sheaf of algebras; that is, one can multiply local operators defined on the same set, restrict a local operator to a smaller set, and reconstruct a local operator from its restrictions to members of some finite open covering of $U$. This makes it possible to describe any local operator $A \in \Psi^{\text {loc }}(U)$ on a manifold using its full symbols in various coordinate charts. For $A \in \Psi^{\text {loc }}(U)$, one can define its wavefront set $\mathrm{WF}_{h}(A)$ as follows: $\left(x_{0}, \xi_{0}\right) \notin$ $\mathrm{WF}_{h}(A)$ if and only if the full symbol of $A$ is $O\left(h^{\infty}\right)$ in some neighborhood of $\left(x_{0}, \xi_{0}\right)$. If $A \in \Psi^{k}$ represents $\widetilde{A} \in \Psi^{\text {loc }}$, then $\mathrm{WF}_{h}(A)=\mathrm{WF}_{h}(\widetilde{A})$; in general, wavefront sets of local operators obey $\mathrm{WF}_{h}(A+B) \subset \mathrm{WF}_{h}(A) \cup \mathrm{WF}_{h}(B)$ and $\mathrm{WF}_{h}(A B) \subset \mathrm{WF}_{h}(A) \cap \mathrm{WF}_{h}(B)$.

Finally, we study microlocalization of arbitrary operators. Let $M_{1}$ and $M_{2}$ be two manifolds. An $h$-dependent family of (properly supported) operators $A(h): C^{\infty}\left(M_{1}\right) \rightarrow \mathcal{D}^{\prime}\left(M_{2}\right)$ is called tempered, or polynomially bounded, if for every compact $K_{1} \subset M_{1}$, there exist $N$ and $C$ such that for any $u \in C_{0}^{\infty}\left(K_{1}\right)$, $\|A(h) u\|_{H_{h}^{-N}\left(M_{2}\right)} \leq C h^{-N}\|u\|_{H_{h}^{N}}$. Note that the composition of a tempered operator with an element of $\Psi^{k}$ is still tempered. We can also treat distributions on $M_{2}$ as operators from a singleton to $M_{2}$.

For a tempered family $A(h)$, we define its wavefront set $\mathrm{WF}_{h}(A) \subset$ $T^{*}\left(M_{1} \times M_{2}\right)$ as follows: $(x, \xi ; y, \eta) \notin \mathrm{WF}_{h}(A)$, if and only if there exist neighborhoods $U_{1}(x, \xi)$ and $U_{2}(y, \eta)$ such that for every $B_{j} \in \Psi^{\operatorname{comp}}\left(M_{j}\right)$ with $\mathrm{WF}_{h}\left(B_{j}\right) \subset U_{j}$, we have $B_{2} A(h) B_{1} \in h^{\infty} \Psi^{-\infty}$. We say that $A_{1}=A_{2}$ microlocally in some open and bounded $U \subset T^{*} M$, if $\mathrm{WF}_{h}\left(A_{1}-A_{2}\right) \cap U=\emptyset$. Also, $A(h)$ is said to be compactly microlocalized, if there exist $C_{j} \in \Psi^{\operatorname{comp}}\left(M_{j}\right)$ such that $A(h)-C_{2} A(h) C_{1} \in h^{\infty} \Psi^{-\infty}$. In this case, all operator norms $\|A(h)\|_{H_{h}^{N_{1}} \rightarrow H_{h}^{N_{2}}}$ are equivalent modulo $O\left(h^{\infty}\right)$; if any of these norms is $O\left(h^{r}\right)$ for some constant $r$, we write $\|A(h)\|=O\left(h^{r}\right)$. Here are some properties:

- If $A$ is compactly microlocalized, then $\mathrm{WF}_{h}(A)$ is compact. The converse, however, need not be true.

- If $A \in \Psi^{k}(M)$, then the two definitions of compact microlocalization of $A$ (via its symbol and as a tempered family of operators) agree; the wavefront set of $A$ as a tempered family of operators is just $\{(x, \xi ; x, \xi) \mid$ $\left.(x, \xi) \in \mathrm{WF}_{h}(A)\right\}$.

- If $A_{1}, A_{2}$ are two tempered operators and at least one of them is either compactly microlocalized or pseudodifferential, then the product $A_{2} A_{1}$ is a tempered operator, and

$$
\begin{aligned}
& \mathrm{WF}_{h}\left(A_{2} A_{1}\right) \subset \mathrm{WF}_{h}\left(A_{1}\right) \circ \mathrm{WF}_{h}\left(A_{2}\right) \\
& \quad=\left\{(x, \xi ; z, \zeta) \mid \exists(y, \eta):(x, \xi ; y, \eta) \in \mathrm{WF}_{h}\left(A_{1}\right),(y, \eta ; z, \zeta) \in \mathrm{WF}_{h}\left(A_{2}\right)\right\} .
\end{aligned}
$$

Moreover, if both $A_{1}, A_{2}$ are compactly microlocalized, so is $A_{2} A_{1}$.

Let us quote the following microlocalization fact for oscillatory integrals, which is the starting point for the construction of semiclassical Fourier integral operators used in Sect. 2.3: 
Proposition 2.1. Assume that $M$ is a manifold, $U \subset M \times \mathbb{R}^{m}$ is open, $\varphi(x, \theta)$ is a smooth real-valued function on $U$, with $x \in M$ and $\theta \in \mathbb{R}^{m}$, and $a(x, \theta) \in$ $C_{0}^{\infty}(U)$. Then, the family of distributions

$$
u(x)=\int_{\mathbb{R}^{m}} e^{i \varphi(x, \theta) / h} a(x, \theta) \mathrm{d} \theta
$$

is compactly microlocalized and

$$
\mathrm{WF}_{h}(u) \subset\left\{\left(x, \partial_{x} \varphi(x, \theta)\right) \mid(x, \theta) \in \operatorname{supp} a, \partial_{\theta} \varphi(x, \theta)=0\right\} .
$$

\subsection{Ellipticity and Formal Functional Calculus}

Assume that $U \subset T^{*} M$ is open and $A \in \Psi_{\mathrm{cl}}^{\text {loc }}(U)$. We say that $A$ is (semiclassically) elliptic on $U$ if its principal symbol does not vanish on $U$. Under this condition, there exists unique operator $A^{-1} \in \Psi_{\mathrm{cl}}^{\text {loc }}(U)$ such that $A^{-1} A, A A^{-1}=I$ as local operators. The next proposition provides the form of the symbol of $A^{-1}$; it is based on the standard parametrix construction:

Proposition 2.2. Fix a coordinate system on $M$. Assume that $A \in \Psi_{\mathrm{cl}}^{\mathrm{loc}}(U)$ is elliptic and has the full symbol $a \sim a_{0}+h a_{1}+h^{2} a_{2}+\cdots$. Then, $A^{-1}$ has the full symbol $b \sim b_{0}+h b_{1}+h^{2} b_{2}+\cdots$, where each $b_{j}$ is a linear combination with constant coefficients of the terms of the form

$$
a_{0}^{-M-1} \prod_{m=1}^{M} \partial_{x}^{\alpha_{m}} \partial_{\xi}^{\beta_{m}} a_{l_{m}} .
$$

Here the (multi)indices $\alpha_{m}, \beta_{m}, l_{m}$ satisfy the condition

$$
\sum_{m=1}^{M}\left|\alpha_{m}\right|=\sum_{m=1}^{M}\left|\beta_{m}\right|=j-\sum_{m=1}^{M} l_{m} .
$$

Furthermore, we can assume that $\left|\alpha_{m}\right|+\left|\beta_{m}\right|+l_{m}>0$ for all $m$.

Proof. We call (2.2) an expression of type $\left(M_{\alpha}, M_{\beta}, L\right)$, where $M_{\alpha}, M_{\beta}, L$ are the sums of $\left|\alpha_{m}\right|,\left|\beta_{m}\right|$, and $l_{m}$, respectively. If $f$ is an expression of type $\left(M_{\alpha}, M_{\beta}, L\right)$, then we can prove by induction that $\partial_{x}^{\alpha} \partial_{\xi}^{\beta} f$ is an expression of type $\left(M_{\alpha}+|\alpha|, M_{\beta}+|\beta|, L\right)$. Now, we write the equation $a \# b=1$; the principal term gives $b_{0}=a_{0}^{-1}$, and the next terms give that each $b_{j}$ is the sum of expressions of type $(j-L, j-L, L)$, by induction and the formula for the symbol product $a \# b$.

Let $A \in \Psi_{\mathrm{cl}}^{k}$; we say that it is elliptic on an open conic at infinity $U \subset T^{*} M$ in the class $\Psi^{k}$ (or microlocally elliptic), if its principal symbol $a_{0}$ satisfies $\left|a_{0}(x, \xi)\right| \geq\langle\xi\rangle^{k} / C(K)$ for $(x, \xi)$ in any given closed conic at infinity $K \subset U$ with compact projection onto $M$, and some constant $C(K)$ depending on $K$. In this case, the full symbol of $A^{-1}$ satisfies the decay conditions of the class $\Psi^{-k}$ in $U$. In particular, if $A \in \Psi_{\mathrm{cl}}^{k}$ is elliptic in the class $\Psi^{k}$ everywhere, then we can define $A^{-1} \in \Psi_{\mathrm{cl}}^{-k}$ for $h$ small enough.

We now construct functional calculus of local real principal pseudodifferential operators. For this, we use holomorphic functional calculus 
[25, Section 7.3]; another approach would be via almost analytic continuation [21, Chapter 8]. First, assume that $A \in \Psi_{\mathrm{cl}}^{\text {comp }}(M)$ has compactly supported Schwartz kernel. In particular, the principal symbol $a_{0}$ of $A$ is compactly supported; let $K \subset \mathbb{C}$ be the image of $a_{0}$. Let $f(z)$ be holomorphic in a neighborhood $\Omega$ of $K$, and let $\gamma \subset \Omega$ be a contour such that $K$ lies inside of $\gamma$. For each $h$, the operator $A$ is bounded $L^{2} \rightarrow L^{2}$; for $h$ small enough, its spectrum lies inside of $\gamma$. Then, we can define the operator $f(A)$ by the formula

$$
f(A)=\frac{1}{2 \pi i} \oint_{\gamma} f(z)(z-A)^{-1} \mathrm{~d} z .
$$

For $z \in \gamma$, the operator $z-A$ is elliptic in the class $\Psi^{0}$; therefore, $(z-A)^{-1} \in$ $\Psi_{\mathrm{cl}}^{0}(M)$. It follows that $f(A) \in \Psi_{\mathrm{cl}}^{0}(M)$. By Proposition 2.2, the full symbol of $f(A)$ (in any coordinate system) is the asymptotic sum

$$
\sum_{j=0}^{\infty} h^{j} \sum_{M=0}^{2 j} f^{(M)}\left(a_{0}(x, \xi)\right) b_{j M}(x, \xi) .
$$

Here $a \sim a_{0}+h a_{1}+\cdots$ is the full symbol of $A ; b_{j M}$ are the functions resulting from applying certain nonlinear differential operators to $a_{0}, a_{1}, \ldots$.

Now, assume that $U \subset T^{*} M$ is open and $A \in \Psi_{\mathrm{cl}}^{\mathrm{loc}}(U)$ has real-valued principal symbol $a_{0}$. Then, the formula $(2.3)$ can be used to define an operator $f[A] \in \Psi_{\mathrm{cl}}^{\text {loc }}(U)$ for any $f \in C^{\infty}(\mathbb{R})$. Note that the principal symbol of $(z-A)^{-1}$ is $\left(z-a_{0}\right)^{-1}$; therefore, the principal symbol of $f[A]$ is $f \circ a_{0}$. The constructed operation possesses the following properties of functional calculus:

Proposition 2.3. Assume that $U \subset T^{*} M$ is open, $A \in \Psi_{\mathrm{cl}}^{\mathrm{loc}}(U)$, and $f, g \in$ $C^{\infty}(\mathbb{R})$. Then:

1. $\mathrm{WF}_{h}(f[A]) \subset a_{0}^{-1}(\operatorname{supp} f)$, where $a_{0}$ is the principal symbol of $A$.

2. If $f(t)=\sum_{j=0}^{K} f_{j} t^{j}$ is a polynomial, then $f[A]=f(A)$, where $f(A)=$ $\sum_{j} f_{j} A^{j}$

3. $(f+g)[A]=f[A]+g[A]$ and $(f g)[A]=f[A] g[A]$.

4. If $B \in \Psi_{\mathrm{cl}}^{\mathrm{loc}}(U)$ and $[A, B]=0$, then $[f[A], B]=0$.

The identities in parts 2-4 are equalities of local operators; in particular, they include the $h^{\infty} \Psi^{-\infty}$ error. In fact, the operator $f[A]$ is only defined uniquely modulo $h^{\infty} \Psi^{-\infty}$.

Proof. 1. Follows immediately from (2.3).

2. Take an open set $V$ compactly contained in $U$; then, there exists $\widetilde{A} \in$ $\Psi_{\mathrm{cl}}^{\mathrm{comp}}(M)$ such that $A=\widetilde{A}$ microlocally on $V$. Since $f$ is entire and $\widetilde{A}$ is compactly microlocalized, we can define $f(\widetilde{A})$ by means of holomorphic functional calculus; it is be a pseudodifferential operator representing $f[\widetilde{A}]$. Now, $f(A)=f(\widetilde{A})$ microlocally on $V$ by properties of multiplication of pseudodifferential operators and $f[A]=f[\widetilde{A}]$ microlocally on $V$ by (2.3); therefore, $f(A)=f[A]$ microlocally on $V$. Since $V$ was arbitrary, we have $f(A)=f[A]$ microlocally on the whole $U$. 
3. We only prove the second statement. It suffices to show that for every coordinate system on $M$, the full symbols of $(f g)[A]$ and $f[A] g[A]$ are equal. However, the terms in the asymptotic decomposition of the full symbol of $(f g)[A]-f[A] g[A]$ at $(x, \xi)$ only depend on the derivatives of the full symbol of $A$ at $(x, \xi)$ and the derivatives of $f$ and $g$ at $a_{0}(x, \xi)$. Therefore, it suffices to consider the case when $f$ and $g$ are polynomials. In this case, we can use the previous part of the proposition and the fact that $f(A) g(A)=(f g)(A)$.

4. This is proven similarly to the previous part, using the fact that $[A, B]=0$ yields $[f(A), B]=0$ for every polynomial $f$.

Finally, under certain conditions on the growth of $f$ and the symbol of $A$ at infinity, $f[A]$ is a globally defined operator:

Proposition 2.4. Assume that $A \in \Psi_{\mathrm{cl}}^{k}(M)$, with $k \geq 0$, and that $A$ is elliptic in the class $\Psi^{k}$ outside of a compact subset of $T^{*} M$. Also, assume that $f \in C^{\infty}(\mathbb{R})$ is a symbol of order $s$, in the sense that for each $l$, there exists a constant $C_{l}$ such that

$$
\left|f^{(l)}(t)\right| \leq C_{l}\langle t\rangle^{s-l}, \quad t \in \mathbb{R} .
$$

Then, $f[A]$ is represented by an operator in $\Psi_{\mathrm{cl}}^{s k}(M)$.

Proof. We use (2.3); by Proposition 2.2, the symbol $b_{j M}$ lies in $S^{k M-j}$. Since $f$ is a symbol of order $s, f^{(M)}$ is a symbol of order $s-M$. Then, since $a_{0} \in S^{k}$ is elliptic outside of a compact set and $k \geq 0$, we have $f^{(M)} \circ a_{0} \in S^{k(s-M)}$. It follows that each term in (2.3) lies in $S^{s \bar{k}-j}$; therefore, this asymptotic sum gives an element of $\Psi_{\mathrm{cl}}^{s k}$.

\subsection{Quantizing Canonical Transformations}

Assume that $M_{1}$ and $M_{2}$ are two manifolds of the same dimension. Recall that the symplectic form $\omega_{j}^{S}$ on $T^{*} M_{j}$ is given by $\omega_{j}^{S}=d \sigma_{j}^{S}$, where $\sigma_{j}^{S}=\xi d x$ is the canonical 1-form. We let $K_{j} \subset T^{*} M_{j}$ be compact and assume that $\Phi: T^{*} M_{1} \rightarrow T^{*} M_{2}$ is a symplectomorphism defined in a neighborhood of $K_{1}$ and such that $\Phi\left(K_{1}\right)=K_{2}$. Then, the form $\sigma_{1}^{S}-\Phi^{*} \sigma_{2}^{S}$ is closed; we say that $\Phi$ is an exact symplectomorphism if this form is exact. Define the classical action over a closed curve in $T^{*} M_{j}$ as the integral of $\sigma_{j}^{S}$ over this curve; then, $\Phi$ is exact if and only if for each closed curve $\gamma$ in the domain of $\Phi$, the classical action over $\gamma$ is equal to the classical action over $\Phi \circ \gamma$. We can quantize exact symplectomorphisms as follows:

Proposition 2.5. Assume that $\Phi$ is an exact symplectomorphism. Then, there exist $h$-dependent families of operators

$$
B_{1}: \mathcal{D}^{\prime}\left(M_{1}\right) \rightarrow C_{0}^{\infty}\left(M_{2}\right), B_{2}: \mathcal{D}^{\prime}\left(M_{2}\right) \rightarrow C_{0}^{\infty}\left(M_{1}\right)
$$

such that:

1. Each $B_{j}$ is compactly microlocalized and has operator norm $O(1)$; moreover, $\mathrm{WF}_{h}\left(B_{1}\right)$ is contained in the graph of $\Phi$ and $\mathrm{WF}_{h}\left(B_{2}\right)$ is contained in the graph of $\Phi^{-1}$. 
2. The operators $B_{1} B_{2}$ and $B_{2} B_{1}$ are equal to the identity microlocally near $K_{2} \times K_{2}$ and $K_{1} \times K_{1}$, respectively.

3. For each $P \in \Psi_{\mathrm{cl}}\left(M_{1}\right)$, there exists $Q \in \Psi_{\mathrm{cl}}\left(M_{2}\right)$ that is intertwined with $P$ via $B_{1}$ and $B_{2}$ :

$$
B_{1} P=Q B_{1}, \quad P B_{2}=B_{2} Q
$$

microlocally near $K_{1} \times K_{2}$ and $K_{2} \times K_{1}$, respectively. Similarly, for each $Q \in \Psi_{\mathrm{cl}}\left(M_{2}\right)$ there exists $P \in \Psi_{\mathrm{cl}}\left(M_{1}\right)$ intertwined with it. Finally, if $P$ and $Q$ are intertwined via $B_{1}$ and $B_{2}$ and $p$ and $q$ are their principal symbols, then $p=q \circ \Phi$ near $K_{1}$.

If the properties $1-3$ hold, we say that the pair $\left(B_{1}, B_{2}\right)$ quantizes the canonical transformation $\Phi$ near $K_{1} \times K_{2}$.

Proof. We take $B_{1}, B_{2}$ to be semiclassical Fourier integral operators associated with $\Phi$ and $\Phi^{-1}$, respectively; their symbols are taken compactly supported and elliptic in a neighborhood of $K_{1} \times K_{2}$. The existence of globally defined elliptic symbols follows from the exactness of $\Phi$; the rest follows from calculus of Fourier integral operators. See [30, Chapter 8] or [51, Chapter 2] for more details.

Note that the operators $B_{1}$ and $B_{2}$ quantizing a given canonical transformation are not unique. In fact, if $X_{j} \in \Psi_{\mathrm{cl}}^{\mathrm{comp}}\left(M_{j}\right)$ are elliptic near $K_{j}$ and $Y_{j} \in \Psi_{\mathrm{cl}}^{\mathrm{comp}}\left(M_{j}\right)$ are their inverses near $K_{j}$, then $\left(X_{2} B_{1} X_{1}, Y_{1} B_{2} Y_{2}\right)$ also quantizes $\Phi$; moreover, $P$ is intertwined with $Q$ via the new pair of operators if and only if $X_{1} P Y_{1}$ is intertwined with $Y_{2} Q X_{2}$ via $\left(B_{1}, B_{2}\right)$.

We now study microlocal properties of Schrödinger propagators. Take $A \in \Psi_{\mathrm{cl}}^{\mathrm{comp}}(M)$ with compactly supported Schwartz kernel and let $a_{0}$ be its principal symbol; we assume that $a_{0}$ is real-valued. In this case the Hamiltonian flow $\exp \left(t H_{a_{0}}\right), t \in \mathbb{R}$, is a family of symplectomorphisms defined on the whole $T^{*} M$; it is the identity outside of $\operatorname{supp} a_{0}$. Moreover, $\exp \left(t H_{a_{0}}\right)$ is exact; indeed, if $V=H_{a_{0}}$, then by Cartan's formula

$$
\mathcal{L}_{V} \sigma^{S}=d\left(i_{V} \sigma^{S}\right)+i_{V} d\left(\sigma^{S}\right)=d\left(i_{V} \sigma^{S}-a_{0}\right)
$$

is exact. Therefore,

$$
d_{t} \exp (t V)^{*} \sigma^{S}=\exp (t V)^{*} \mathcal{L}_{V} \sigma^{S}
$$

is exact and $\exp (t V)^{*} \sigma^{S}-\sigma^{S}$ is exact for all $t$.

For each $t$, define the operator $\exp (i t A / h)$ as the solution to the Schrödinger equation

$$
h D_{t} \exp (i t A / h)=A \exp (i t A / h)=\exp (i t A / h) A
$$

in the algebra of bounded operators on $L^{2}(M)$, with the initial condition $\exp (i 0 A / h)=I$. Such a family exists since $A$ is a bounded operator on $L^{2}(M)$ for all $h$. Here are some of its properties (see also [54, Chapter 10]):

Proposition 2.6. $\quad$ 1. The operator $\exp (i t A / h)-I$ is compactly microlocalized and has operator norm $O(1)$. 
2. If $A, B \in \Psi_{\mathrm{cl}}^{\mathrm{comp}}$ have real-valued principal symbols and $[A, B]=O\left(h^{\infty}\right)$, then

$$
\begin{aligned}
{[\exp (i t A / h), B] } & =O\left(h^{\infty}\right), \exp (i t(A+B) / h) \\
& =\exp (i t A / h) \exp (i t B / h)+O\left(h^{\infty}\right) .
\end{aligned}
$$

(We do not specify the functional spaces as the estimated families of operators are compactly microlocalized, so all Sobolev norms are equivalent.) In particular, if $B=O\left(h^{\infty}\right)$, then the propagators of $A$ and $A+B$ are the same modulo $O\left(h^{\infty}\right)$.

3. Let $P \in \Psi_{\mathrm{cl}}$ and take

$$
P_{t}=\exp (i t A / h) P \exp (-i t A / h) .
$$

Then, $P_{t}$ is pseudodifferential and its full symbol depends smoothly on $t$. The principal symbol of $P_{t}$ is $p_{0} \circ \exp \left(t H_{a_{0}}\right)$, where $p_{0}$ is the principal symbol of $P$; moreover,

$$
\mathrm{WF}_{h}\left(P_{t}\right)=\exp \left(-t H_{a_{0}}\right)\left(\mathrm{WF}_{h}(P)\right) .
$$

4. Let $K \subset T^{*} M$ be a compact set invariant under the Hamiltonian flow of $a_{0}$. If $X \in \Psi_{\mathrm{cl}}^{\mathrm{comp}}$ is equal to the identity microlocally near $K$, then the pair $(X \exp (-i t A / h), X \exp (i t A / h))$ quantizes the canonical transformation $\exp \left(t H_{a_{0}}\right)$ near $K \times K$. Moreover, if $P, Q \in \Psi_{\mathrm{cl}}^{\mathrm{comp}}$ are intertwined via these two operators, then $Q=\exp (-i t A / h) P \exp (i t A / h)$ microlocally near $K$.

5. Assume that $V$ is a compactly supported vector field on $M$, and let $\exp (t V): M \rightarrow M$ be the corresponding flow, defined for all $t$; denote by $\exp (t V)^{*}$ the pull-back operator, acting on functions on $M$. Let $K \subset T^{*} M$ be compact and invariant under the flow of $V$, and $X \in \Psi_{\mathrm{cl}}^{\mathrm{comp}}$ have realvalued principal symbol and be equal to the identity microlocally near $K$; consider $(h V / i) X \in \Psi_{\mathrm{cl}}^{\mathrm{comp}}$. Then, for each $t$,

$$
\exp (i t(h V / i) X / h)=\exp (t V)^{*}
$$

microlocally near $K \times K$.

The statements above are true locally uniformly in $t$.

Proof. 1. First, take $u \in L^{2}(M)$; then, since the principal symbol of $A$ is real-valued, we have $\left\|A-A^{*}\right\|_{L^{2} \rightarrow L^{2}}=O(h)$ and thus

$$
\begin{aligned}
D_{t}\|\exp (i t A / h) u\|_{L^{2}}^{2} & =h^{-1}\left(\left(A-A^{*}\right) \exp (i t A / h) u, \exp (i t A / h) u\right)_{L^{2}} \\
& =O\left(\|\exp (i t A / h) u\|_{L^{2}}^{2}\right) ;
\end{aligned}
$$

therefore, $\exp (i t A / h)$ is tempered:

$$
\|\exp (i t A / h)\|_{L^{2} \rightarrow L^{2}}=O\left(e^{C|t|}\right) .
$$

The rest follows from the identity

$$
\exp (i t A / h)=I+\frac{i t}{h} A+\frac{i}{h} A \int_{0}^{t}(t-s) \exp (i s A / h) \mathrm{d} s \cdot \frac{i}{h} A .
$$


2. We have

$$
\begin{aligned}
D_{t}(\exp (i t A / h) B \exp (-i t A / h)) & =h^{-1} \exp (i t A / h)[A, B] \exp (-i t A / h) \\
& =O\left(h^{\infty}\right) ;
\end{aligned}
$$

this proves the first identity. The second one is proved in a similar fashion:

$$
D_{t}(\exp (-i t(A+B) / h) \exp (i t A / h) \exp (i t B / h))=O\left(h^{\infty}\right) .
$$

3. We construct a family $\widetilde{P}_{t}$ of classical pseudodifferential operators, each equal to $P$ microlocally outside of a compact set, solving the initial-value problem

$$
D_{t} \widetilde{P}_{t}=h^{-1}\left[A, \widetilde{P}_{t}\right]+O\left(h^{\infty}\right), \widetilde{P}_{0}=P+O\left(h^{\infty}\right) .
$$

For that, we can write a countable system of equations on the components of the full symbol of $\widetilde{P}_{t}$. In particular, if $p(t)$ is the principal symbol of $\widetilde{P}_{t}$, we get

$$
\partial_{t} p(t)=\left\{a_{0}, p(t)\right\}=H_{a_{0}} p(t) ;
$$

it follows that $p(t)=p_{0} \circ \exp \left(t H_{a_{0}}\right)$. Similarly we can recover the wavefront set of $\widetilde{P}_{t}$ from that of $P$. Now,

$$
\partial_{t}\left(\exp (-i t A / h) \widetilde{P}_{t} \exp (i t A / h)\right)=O\left(h^{\infty}\right) ;
$$

therefore, $P_{t}=\widetilde{P}_{t}+O\left(h^{\infty}\right)$.

4. Since $X$ is compactly microlocalized, so are the operators $B_{1}=$ $X \exp (-i t A / h)$ and $B_{2}=X \exp (i t A / h)$. Next, if $Y_{2}, Y_{1} \in \Psi_{\mathrm{cl}}^{\mathrm{comp}}$, then

$$
Y_{2} B_{1} Y_{1}=Y_{2} X\left(\exp (-i t A / h) Y_{1} \exp (i t A / h)\right) \exp (-i t A / h) ;
$$

using our knowledge of the wavefront set of the operator in brackets, we see that this is $O\left(h^{\infty}\right)$ if

$$
\mathrm{WF}_{h}\left(Y_{2}\right) \cap \exp \left(t H_{a_{0}}\right) \mathrm{WF}_{h}\left(Y_{1}\right)=\emptyset .
$$

Therefore, $\mathrm{WF}_{h}\left(B_{1}\right)$ is contained in the graph of $\exp \left(t H_{a_{0}}\right)$; similarly, $\mathrm{WF}_{h}\left(B_{2}\right)$ is contained in the graph of $\exp \left(-t H_{a_{0}}\right)$. Next,

$$
B_{1} B_{2}=X(\exp (-i t A / h) X \exp (i t A / h)) ;
$$

however, the operator in brackets is the identity microlocally near $K$, as $X$ is the identity microlocally near $K$ and $K$ is invariant under $\exp \left(t H_{a_{0}}\right)$. Therefore, $B_{1} B_{2}$ is the identity microlocally near $K$. The intertwining property is proved in a similar fashion.

5. We have

$$
\partial_{t}\left(\exp (t V X) \exp (-t V)^{*}\right)=\exp (t V X) V(X-I) \exp (-t V)^{*}=O\left(h^{\infty}\right)
$$

microlocally near $K \times K$.

Finally, we consider the special case $a_{0}=0$; in other words, we study $\exp (i t A)$, where $A \in \Psi_{\mathrm{cl}}^{\mathrm{comp}}$. Since the associated canonical transformation is the identity, it is not unexpected that $\exp (i t A)$ is a pseudodifferential operator: 
Proposition 2.7. Let $a_{1}$ be the principal symbol of $A$. Then:

1. $\exp (i t A)-I \in \Psi_{\mathrm{cl}}^{\mathrm{comp}}$ and the principal symbol of $\exp (i t A)$ equals $e^{\text {ita }_{1}}$. Moreover, if $A_{1}=A_{2}$ microlocally in some open set, then $\exp \left(\right.$ it $\left.A_{1}\right)=$ $\exp \left(i t A_{2}\right)$ microlocally in the same set.

2. For any $P \in \Psi_{\mathrm{cl}}^{\mathrm{comp}}$, we have the following asymptotic sum:

$$
\exp (i t A) P \exp (-i t A) \sim \sum_{j \geq 0} \frac{\left(i t \operatorname{ad}_{A}\right)^{j} P}{j !},
$$

where $\operatorname{ad}_{A} Q=[A, Q]$ for every $Q$.

3. If $U \subset T^{*} M$ is connected and $\exp (i A)=I$ microlocally in $U$, then $A=2 \pi l$ microlocally in $U$, where $l$ is an integer constant.

Proof. 1. We can find a family of pseudodifferential operators $B_{t}$ solving

$$
\partial_{t} B_{t}=i A B_{t}+O\left(h^{\infty}\right), \quad B_{0}=I,
$$

by subsequently finding each member of the asymptotic decomposition of the full symbol of $B_{t}$. Then

$$
\partial_{t}\left(\exp (-i t A) B_{t}\right)=O\left(h^{\infty}\right) ;
$$

therefore, $\exp (i t A)=B_{t}+O\left(h^{\infty}\right)$. The properties of $B_{t}$ can be verified directly.

2. Follows directly from the equation

$$
\partial_{t}(\exp (i t A) P \exp (-i t A))=i \operatorname{ad}_{A}(\exp (i t A) P \exp (-i t A)) .
$$

3. By calculating the principal symbol of $\exp (i A)$, we see that $a_{1}$ has to be equal to $2 \pi l$ in $U$ for some constant $l \in \mathbb{Z}$. Subtracting this constant, we reduce to the case when $A=O(h)$. However, if $A=O\left(h^{N}\right)$ for some $N \geq 1$, then $\exp (i A)=I+i A+O\left(h^{N+1}\right)$; by induction, we get $A=O\left(h^{N}\right)$ microlocally in $U$ for all $N$.

\subsection{Integrable Systems}

Assume that $M$ is a two-dimensional manifold and $p_{1}, p_{2}$ are two real-valued functions defined on an open set $U \subset T^{*} M$ such that:

- $\left\{p_{1}, p_{2}\right\}=0$;

- for $\mathbf{p}=\left(p_{1}, p_{2}\right): U \rightarrow \mathbb{R}^{2}$ and each $\rho \in \mathbf{p}(U)$, the set $\mathbf{p}^{-1}(\rho)$ is compact and connected.

We call such $\mathbf{p}$ an integrable system. Note that if $V \subset \mathbb{R}^{2}$ is open and intersects $\mathbf{p}(U)$, and $F: V \rightarrow \mathbb{R}^{2}$ is a diffeomorphism onto its image, then $F(\mathbf{p})$ is an integrable system on $\mathbf{p}^{-1}(V)$.

We say that an integrable system $\mathbf{p}: U \rightarrow \mathbb{R}^{2}$ is nondegenerate on $U$, if the differentials of $p_{1}$ and $p_{2}$ are linearly independent everywhere on $U$. The following two propositions describe the normal form for nondegenerate integrable systems:

Proposition 2.8. Assume that the integrable system $\mathbf{p}$ is nondegenerate on $U$. Then: 
1. For each $\rho \in \mathbf{p}(U)$, the set $\mathbf{p}^{-1}(\rho) \subset T^{*} M$ is a Lagrangian torus. Moreover, the family of diffeomorphisms

$$
\phi_{t}=\exp \left(t_{1} H_{p_{1}}+t_{2} H_{p_{2}}\right), \quad t=\left(t_{1}, t_{2}\right) \in \mathbb{R}^{2},
$$

defines a transitive action of $\mathbb{R}^{2}$ on $\mathbf{p}^{-1}(\rho)$. The kernel of this action is a rank two lattice depending smoothly on $\rho$; we call it the periodicity lattice (at $\rho)$.

2. For each $\rho_{0} \in \mathbf{p}(U)$, there exists a neighborhood $V\left(\rho_{0}\right)$ and a diffeomorphism $F: V \rightarrow \mathbb{R}^{2}$ onto its image such that the nondegenerate integrable system $F(\mathbf{p})$ has periodicity lattice $2 \pi \mathbb{Z}^{2}$ at every point. Moreover, if the Hamiltonian flow of $p_{2}$ is periodic with minimal period $2 \pi$, we can take the second component of $F(\mathbf{p})$ to be $p_{2}$.

3. Assume that $V \subset \mathbf{p}(U)$ is open and connected and $F_{1}, F_{2}: V \rightarrow \mathbb{R}^{2}$ are two maps satisfying the conditions of part 2. Then, there exist $A \in \mathrm{GL}(2, \mathbb{Z})$ and $b \in \mathbb{R}^{2}$ such that $F_{2}=A \cdot F_{1}+b$.

Proof. This is a version of Arnold-Liouville theorem; see [24, Section 1] for the proof.

Proposition 2.9. Assume that $\mathbf{p}: U \rightarrow \mathbb{R}^{2}, \mathbf{p}^{\prime}: U^{\prime} \rightarrow \mathbb{R}^{2}$, are nondegenerate integrable systems with periodicity lattices $2 \pi \mathbb{Z}^{2}$ at every point; here $U \subset T^{*} M$, $U^{\prime} \subset T^{*} M^{\prime}$. Take $\rho_{0} \in \mathbf{p}(U) \cap \mathbf{p}^{\prime}\left(U^{\prime}\right)$. Then:

1. There exists a symplectomorphism $\Phi$ from a neighborhood of $\mathbf{p}^{-1}\left(\rho_{0}\right)$ in $T^{*} M$ onto a neighborhood of $\left(\mathbf{p}^{\prime}\right)^{-1}\left(\rho_{0}\right)$ in $T^{*} M^{\prime}$ such that $\mathbf{p}=\mathbf{p}^{\prime} \circ \Phi$.

2. $\Phi$ is exact, as defined in Sect. 2.3, if and only if

$$
\int_{\gamma_{j}} \sigma^{S}=\int_{\gamma_{j}^{\prime}} \sigma^{S}, \quad j=1,2,
$$

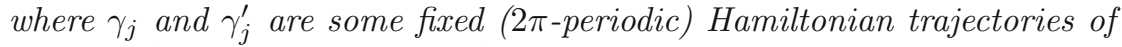
$p_{j}$ on $\mathbf{p}^{-1}\left(\rho_{0}\right)$ and $p_{j}^{\prime}$ on $\left(\mathbf{p}^{\prime}\right)^{-1}\left(\rho_{0}\right)$, respectively.

Proof. Part 1 again follows from Arnold-Liouville theorem. For part 2, we use that the closed 1-form $\sigma^{S}-\Phi^{*} \sigma^{\prime S}$ on a tubular neighborhood of $\mathbf{p}^{-1}\left(\rho_{0}\right)$ is exact if and only if its integral over each $\gamma_{j}$ is zero. Since $\gamma_{j}$ lie in $\mathbf{p}^{-1}\left(\rho_{0}\right)$ and the restriction of $d \sigma^{S}=\omega^{S}$ to $\mathbf{p}^{-1}\left(\rho_{0}\right)$ is zero, we may shift $\gamma_{j}$ to make both of them start at a fixed point $\left(x_{0}, \xi_{0}\right) \in \mathbf{p}^{-1}\left(\rho_{0}\right)$. Similarly, we may assume that both $\gamma_{j}^{\prime}$ start at $\Phi\left(x_{0}, \xi_{0}\right)$. But in this case $\gamma_{j}^{\prime}=\Phi \circ \gamma_{j}$ and

$$
\int_{\gamma_{j}} \sigma^{S}-\Phi^{*} \sigma^{\prime S}=\int_{\gamma_{j}} \sigma^{S}-\int_{\gamma_{j}^{\prime}} \sigma^{\prime S},
$$

which finishes the proof.

Next, we establish normal form for one-dimensional Hamiltonian systems with one degenerate point. For that, consider $\mathbb{R}_{x, \xi}^{2}$ with the standard symplectic form $d \xi \wedge d x$, and define $\zeta=\left(x^{2}+\xi^{2}\right) / 2$; then, $\zeta$ has unique critical point at zero and its Hamiltonian flow is $2 \pi$-periodic. 
Proposition 2.10. Assume that $p(x, \xi)$ is a real-valued function defined on an open subset of $\mathbb{R}^{2}$ and for some $A \in \mathbb{R}$,

- the set $K_{A}=\{p \leq A\}$ is compact;

- $p$ has exactly one critical point $\left(x_{0}, \xi_{0}\right)$ in $K_{A}, p\left(x_{0}, \xi_{0}\right)<A$, and the Hessian of $p$ at $\left(x_{0}, \xi_{0}\right)$ is positive definite.

Then, there exists a smooth function $F$ on the segment $\left[p\left(x_{0}, \xi_{0}\right), A\right]$, with $F^{\prime}>0$ everywhere and $F\left(p\left(x_{0}, \xi_{0}\right)\right)=0$, and a symplectomorphism $\Psi$ from $K_{A}$ onto the disk $\{\zeta \leq F(A)\} \subset \mathbb{R}^{2}$ such that $F(p)=\zeta \circ \Psi$. Moreover, $F^{\prime}\left(p\left(x_{0}, \xi_{0}\right)\right)=\left(\operatorname{det} \nabla^{2} p\left(x_{0}, \xi_{0}\right)\right)^{-1 / 2}$. If $p$ depends smoothly on some parameter $Z$, then $F$ and $\Psi$ can be chosen locally to depend smoothly on this parameter as well.

Proof. Without loss of generality, we may assume that $p\left(x_{0}, \xi_{0}\right)=0$. Recall that in one dimension, symplectomorphisms are diffeomorphisms that preserve both area and orientation. By Morse lemma, there exists an orientation preserving diffeomorphism $\Theta$ from a neighborhood of $\left(x_{0}, \xi_{0}\right)$ onto a neighborhood of the origin such that $p=\zeta \circ \Theta$. Using the gradient flow of $p$, we can extend $\Theta$ to a diffeomorphism from $K_{A}$ to the disk $\{\zeta \leq A\}$ such that $p=\zeta \circ \Theta$. Let $J$ be the Jacobian of $\Theta^{-1}$; then, the integral of $J$ inside the disk $\{\zeta \leq a\}$ is a smooth function of $a$. Therefore, there exists unique function $F$ smooth on $[0, A]$ such that $F^{\prime}>0$ everywhere, $F(0)=0$, and the integral of $J$ inside the disk $\{F(\zeta) \leq a\}$, that is, the area of $\Theta^{-1}(\{F(\zeta) \leq a\})=\{F(p) \leq a\} \subset K_{A}$, is equal to $2 \pi a$.

Let $\widetilde{\Theta}$ be a diffeomorphism from $K_{A}$ onto $\{\zeta \leq F(A)\}$ such that $F(p)=$ $\zeta \circ \widetilde{\Theta}$ (constructed as in the previous paragraph, taking $F(p)$ in place of $p$ ) and let $\tilde{J}$ be the Jacobian of $\widetilde{\Theta}^{-1}$. We know that for $0 \leq a \leq F(A)$, the integral of $\tilde{J}-1$ over $\{\zeta \leq a\}$ is equal to 0 . Introduce polar coordinates $(r, \varphi)$; then, there exists a smooth function $\psi$ such that $\tilde{J}=1+\partial_{\varphi} \psi$ (see Proposition 3.7). The transformation

$$
\widetilde{\Psi}:(r, \varphi) \mapsto(r, \varphi+\psi)
$$

is a diffeomorphism from $\{\zeta \leq F(A)\}$ to itself and has Jacobian $\tilde{J}$; it remains to put $\Psi=\widetilde{\Psi} \circ \widetilde{\Theta}$. To compute $F^{\prime}\left(p_{0}\left(x_{0}, \xi_{0}\right)\right)$, we can compare the Hessians of $F(p)$ and $\zeta \circ \Phi$ at $\left(x_{0}, \xi_{0}\right)$.

The function $F$ is uniquely determined by $p$ and thus will depend smoothly on $Z$. As for $\Psi$, we first note that $\widetilde{\Theta}$ was constructed using Morse lemma and thus can be chosen locally to depend smoothly on $Z$ (see for example [54, Proof of Theorem 3.15]). Next, we can fix $\psi$ by requiring that it integrates to zero over each circle centered at the origin (see Proposition 3.7); then $\psi$, and thus $\widetilde{\Psi}$, will depend smoothly on $Z$.

\section{Angular Problem}

\subsection{Outline of the Proof}

Consider the semiclassical differential operators (using the notation of (1.12)) 


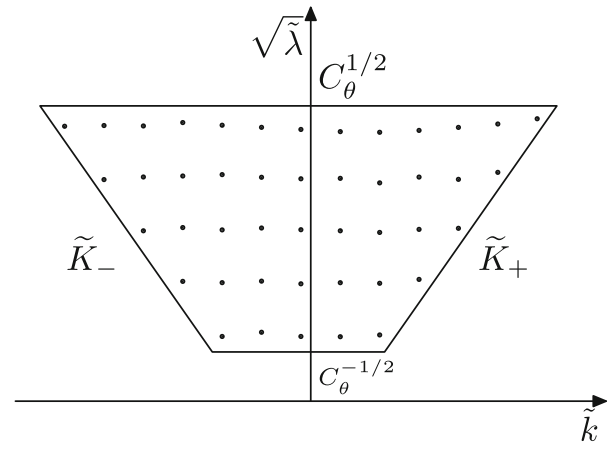

Figure 2. The joint spectrum and the set $\widetilde{K}$

$$
\begin{gathered}
P_{1}(\tilde{\omega}, \tilde{\nu} ; h)=h^{2} P_{\theta}(\omega)=\frac{1}{\sin \theta}\left(h D_{\theta}\right)\left(\Delta_{\theta} \sin \theta \cdot h D_{\theta}\right) \\
+\frac{(1+\alpha)^{2}}{\Delta_{\theta} \sin ^{2} \theta}\left(a(\tilde{\omega}+i h \tilde{\nu}) \sin ^{2} \theta-h D_{\varphi}\right)^{2}, \\
P_{2}(h)=h D_{\varphi}
\end{gathered}
$$

on the sphere $\mathbb{S}^{2}$. Then, $(\omega, \lambda, k)$ is a pole of $R_{\theta}$ if and only if $(\tilde{\lambda}+i h \tilde{\mu}, \tilde{k})$ lies in the joint spectrum of the operators $\left(P_{1}, P_{2}\right)$ (see Definition A.1). For $a=0$, $P_{1}$ is the Laplace-Beltrami operator on the round sphere (multiplied by $-h^{2}$ ); therefore, the joint spectrum of $\left(P_{1}, P_{2}\right)$ is given by the spherical harmonics $(\tilde{l}(\tilde{l}+h), \tilde{k}), \tilde{k}, \tilde{l} \in h \mathbb{Z},|\tilde{k}| \leq \tilde{l}$ (see for example [48, Section 8.4]). In the end of this subsection, we give a short description of which parts of the angular problem are simplified for $a=0$. For general small $a$, we will prove that the joint spectrum is characterized by the following

Proposition 3.1. Let $\tilde{\omega}, \tilde{\nu}$ satisfy (1.15); we suppress dependence of the operators and symbols on these parameters. Consider (Fig. 2)

$$
\begin{gathered}
\tilde{K}=\left\{(\tilde{\lambda}, \tilde{k}) \mid C_{\theta}^{-1} \leq \tilde{\lambda} \leq C_{\theta}, \tilde{\lambda} \geq(1+\alpha)^{2}(\tilde{k}-a \tilde{\omega})^{2}\right\} \subset \mathbb{R}^{2}, \\
\widetilde{K}_{ \pm}=\{(\tilde{\lambda}, \tilde{k}) \in \tilde{K} \mid(1+\alpha)(\tilde{k}-a \tilde{\omega})= \pm \sqrt{\tilde{\lambda}}\} .
\end{gathered}
$$

Then, there exist functions $G_{ \pm}(\tilde{\lambda}, \tilde{k} ; h)$ such that:

1. $G_{ \pm}$is a complex valued classical symbol in $h$, smooth in a fixed neighborhood of $\widetilde{K}$. For $(\tilde{\lambda}, \tilde{k})$ near $\widetilde{K}$ and $|\tilde{\mu}| \leq C_{\theta}$, we can define $G_{ \pm}(\tilde{\lambda}+i h \tilde{\mu}, \tilde{k})$ by means of an asymptotic (analytic) Taylor series for $G_{ \pm}$at $(\tilde{\lambda}, \tilde{k})$.

2. For $a=0, G_{ \pm}(\tilde{\lambda}, \tilde{k} ; h)=-h / 2+\sqrt{\tilde{\lambda}+h^{2} / 4} \mp \tilde{k}$.

3. $G_{-}(\tilde{\lambda}, \tilde{k} ; h)-G_{+}(\tilde{\lambda}, \tilde{k} ; h)=2 \tilde{k}$.

4. Let $F_{ \pm}$be the principal symbol of $G_{ \pm}$. Then, $F_{ \pm}$is real-valued, $\partial_{\tilde{\lambda}} F_{ \pm}>0$ and $\mp \partial_{\tilde{k}} F_{ \pm}>0$ on $\widetilde{K}$, and $\left.F_{ \pm}\right|_{\widetilde{K}_{ \pm}}=0$.

5. For $h$ small enough, the set of elements $(\tilde{\lambda}+i h \tilde{\mu}, \tilde{k})$ of the joint spectrum of $\left(P_{1}, P_{2}\right)$ satisfying (1.15) lies within $O(h)$ of $\widetilde{K}$ and coincides modulo 
$O\left(h^{\infty}\right)$ with the set of solutions to the quantization conditions

$$
\tilde{k} \in h \mathbb{Z}, G_{ \pm}(\tilde{\lambda}+i h \tilde{\mu}, \tilde{k}) \in h \mathbb{N} ;
$$

here $\mathbb{N}$ is the set of nonnegative integers. Note that the conditions $G_{+} \in \mathbb{Z}$ and $G_{-} \in \mathbb{Z}$ are equivalent; however, we also require that both $G_{+}$and $G_{-}$be nonnegative. Moreover, the corresponding joint eigenspaces are one dimensional.

Proposition 1.6 follows from the proof of Proposition 3.1. In fact, the symbol $\mathcal{F}^{\theta}(\tilde{l}, \tilde{\omega}, \tilde{\nu}, \tilde{k} ; h)$ is defined as the solution $\tilde{\lambda}+i h \tilde{\mu}$ to the equation

$$
G_{+}(\tilde{\lambda}+i h \tilde{\mu}, \tilde{k}, \tilde{\omega}+i h \tilde{\nu} ; h)=\tilde{l}-\tilde{k} ;
$$

this proves part (1) of Definition 1.1. The resolvent estimates are an immediate corollary of the ones stated in Proposition 3.8 below. The decomposition of $\mathcal{F}_{0}^{\theta}$ at $a=0$ follows from Proposition 3.4.

We now give the schema of the proof of Proposition 3.1. Let $p_{j 0}$ be the principal symbol of $P_{j}$; note that both $p_{10}$ and $p_{20}$ are real-valued; also, define $\mathbf{p}=\left(p_{10}, p_{20}\right): T^{*} \mathbb{S}^{2} \rightarrow \mathbb{R}^{2}$. In Sect. 3.2, we construct the principal parts $F_{ \pm}$of the quantization symbols globally in $\widetilde{K}$ and show that the intersection of the image of $\mathbf{p}$ with $\left\{C_{\theta}^{-1} \leq \tilde{\lambda} \leq C_{\theta}\right\}$ is exactly $\widetilde{K}$. Using the theory of integrable systems described in Sect. 2.4, we then construct local symplectomorphisms conjugating $\left(F_{ \pm}(\mathbf{p}), p_{20}\right)$ away from $\widetilde{K}_{\mp}$ to the system $(\zeta, \eta)$ on $T^{*} \mathcal{M}$, where $\mathcal{M}=\mathbb{R}_{x} \times \mathbb{S}_{y}^{1}$ is called the model space, $(\xi, \eta)$ are the momenta corresponding to $(x, y)$, and

$$
\zeta=\frac{x^{2}+\xi^{2}}{2} .
$$

Note that the integrable system $(\zeta, \eta)$ is nondegenerate on $\{\zeta>0\}$ with periodicity lattice $2 \pi \mathbb{Z}^{2}$, and $d \zeta=0$ on $\{\zeta=0\}$.

Next, we take $\left(\tilde{\lambda}_{0}, \tilde{k}_{0}\right) \in \widetilde{K}$ and show that joint eigenvalues in a certain $h$-independent neighborhood of this point are given by a quantization condition. For this, we first use Egorov's theorem and the symplectomorphisms constructed in Sect. 3.2 to conjugate $P_{1}, P_{2}$ microlocally near $\mathbf{p}^{-1}\left(\tilde{\lambda}_{0}, \tilde{k}_{0}\right)$ to some pseudodifferential operators $Q_{1}, Q_{2}$ on $\mathcal{M}$. The principal symbols of $Q_{j}$ are real-valued functions of $(\zeta, \eta)$ only; in Sect. 3.3, we use Moser averaging to further conjugate $Q_{1}, Q_{2}$ by elliptic pseudodifferential operators so that the full symbols of $Q_{j}$ depend only on $(\zeta, \eta)$. In Sect. 3.4, we use spectral theory to construct a local Grushin problem for $\left(Q_{1}, Q_{2}\right)$, which we can conjugate back to a local Grushin problem for $\left(P_{1}, P_{2}\right)$; then, we can apply the results of Appendix A to obtain local quantization conditions (Proposition 3.8). To pass from these local conditions to the global one, we use

Proposition 3.2. Assume that $G_{j}(\tilde{\lambda}, \tilde{k} ; h)$ are two complex valued classical symbols in $h$ defined in some open set $U \subset \mathbb{R}^{2}$, their principal symbols are both equal to some real-valued $F(\tilde{\lambda}, \tilde{k})$, with $\partial_{\tilde{\lambda}} F \neq 0$ everywhere and $\{F \geq 0\}$ convex, and solution sets to quantization conditions

$$
\tilde{k} \in h \mathbb{Z}, G_{j}(\tilde{\lambda}+i h \tilde{\mu}, \tilde{k}) \in h \mathbb{N}
$$


in the region $(\tilde{\lambda}, \tilde{k}) \in U, \tilde{\mu}=O(1)$ coincide modulo $O\left(h^{\infty}\right)$. Then, $G_{1}-G_{2}=$ $h l+O\left(h^{\infty}\right)$ on $\{F \geq 0\}$ for some constant $l \in \mathbb{Z}$. Moreover, if $\{F=0\} \cap U \neq \emptyset$, then $l=0$.

Proof. Assume that $\left(\tilde{\lambda}_{1}, \tilde{k}_{1}\right) \in\{F \geq 0\}$. Then, for every $h$, there is a solution $(\tilde{\lambda}(h)+i h \tilde{\mu}(h), \tilde{k}(h))$ to the quantization conditions within $O(h)$ of $\left(\tilde{\lambda}_{1}, \tilde{k}_{1}\right)$; we know that

$$
G_{j}(\tilde{\lambda}(h)+i h \tilde{\mu}(h), \tilde{k}(h)) \in h \mathbb{Z}+O\left(h^{\infty}\right), \quad j=1,2,
$$

and thus $\left(G_{1}-G_{2}\right)(\tilde{\lambda}(h)+i h \tilde{\mu}(h), \tilde{k}(h))=h l(h)+O\left(h^{\infty}\right)$, for some $l(h) \in \mathbb{Z}$. Since $G_{1}-G_{2}=O(h)$ in particular in $C^{1}$, we have

$$
\left|\left(G_{1}-G_{2}\right)\left(\tilde{\lambda}_{1}, \tilde{k}_{1}\right)-h l(h)\right|=O\left(h^{2}\right) .
$$

Therefore, $l(h)$ is constant for $h$ small enough and it is equal to the difference of subprincipal symbols of $G_{1}$ and $G_{2}$ at $\left(\tilde{\lambda}_{1}, \tilde{k}_{1}\right)$. It follows that $l(h)$ is independent of $\left(\tilde{\lambda}_{1}, \tilde{k}_{1}\right)$; we can subtract it from one of the symbols to reduce to the case when $G_{1}-G_{2}=O\left(h^{2}\right)$. The analysis in the beginning of this proof then shows that

$$
\left\|G_{1}-G_{2}\right\|_{C(F \geq 0)}=O\left(h\left\|G_{1}-G_{2}\right\|_{C^{1}(F \geq 0)}+h^{\infty}\right) .
$$

Arguing by induction, we get $G_{1}-G_{2}=O\left(h^{N}\right)$ for all $N$. The last statement follows directly by taking solutions to the quantization conditions with $G_{j}=0$ and requiring that they satisfy the quantization conditions $G_{3-j} \geq 0$.

We can now cover $\widetilde{K}$ by a finite family of open sets, on each of which there exists a local quantization condition. Using Proposition 3.2 and starting from $\widetilde{K}_{ \pm}$, we can modify the local quantization conditions and piece them together to get unique (modulo $\left.h^{\infty}\right)$ global $G_{ \pm}$. The joint spectrum of $\left(P_{1}, P_{2}\right)$ in a neighborhood of $\widetilde{K}$ is then given by the global quantization condition; the joint spectrum outside of this neighborhood, but satisfying (1.15), is empty by part 2 of Proposition 3.8.

Also, the principal symbol of $G_{-}-G_{+}$is equal to $2 \tilde{k}$; therefore, $G_{-}-G_{+}-2 \tilde{k}$ is equal to $l \tilde{h}$ for some fixed $l \in \mathbb{Z}$. However, $G_{ \pm}$depend smoothly on $a$ and thus it is enough to prove that $l=0$ for $a=0$; in the latter case, the symbols $G_{ \pm}$are computed explicitly from the spectrum of Laplacian on the round sphere. (Without such a reference point, one would need to analyze the subprincipal symbols of $G_{ \pm}$using the Maslov index). This finishes the proof of Proposition 3.1.

Finally, let us outline the argument in the special case $a=0$ and indicate which parts of the construction are simplified. The formulas below are not used in the general argument; we provide them for the reader's convenience. The principal symbol $p_{10}$ of $P_{1}$ is just the square of the norm on $T^{*} \mathbb{S}^{2}$ generated by the round metric:

$$
p_{10}=\xi_{\theta}^{2}+\frac{\xi_{\varphi}^{2}}{\sin ^{2} \theta} .
$$


The set $\mathbf{p}^{-1}(\tilde{\lambda}, \tilde{k})$ consists of all cotangent vectors with length $\sqrt{\tilde{\lambda}}$ and momentum $\tilde{k}$; therefore

1. for $\tilde{\lambda} \leq \tilde{k}^{2}$ (corresponding to the complement of $\left.\tilde{K}\right)$, the set $\mathbf{p}^{-1}(\tilde{\lambda}, \tilde{k})$ is empty;

2. for $\tilde{k}= \pm \sqrt{\tilde{\lambda}}$ (corresponding to $\widetilde{K}_{ \pm}$), the set $\mathbf{p}^{-1}(\tilde{\lambda}, \tilde{k})$ is a circle, consisting of covectors tangent to the equator with length $\sqrt{\tilde{\lambda}}$ and direction determined by the choice of sign;

3. for $\tilde{\lambda}>\tilde{k}^{2}$ (corresponding to the interior of $\left.\widetilde{K}\right)$, the set $\mathbf{p}^{-1}(\tilde{\lambda}, \tilde{k})$ is a Liouville torus.

The principal parts $F_{ \pm}$of the quantization symbols, constructed in Proposition 3.4, can be computed explicitly: $F_{ \pm}=\sqrt{\tilde{\lambda}} \mp \tilde{k}$ (see the proof of part 2 of this Proposition). Then, $F_{ \pm}^{-1}(\zeta, \eta)=(\zeta \pm \eta)^{2}$. For $\pm \tilde{k}>0$, the canonical transformation $\Phi_{ \pm}$from Proposition 3.5 can be taken in the form

$$
\begin{aligned}
& \left(\theta, \varphi, \xi_{\theta}, \xi_{\varphi}\right) \mapsto(x, y, \xi, \eta) \\
& =\left(\left(2 p_{10}\right)^{1 / 2}\left(\sqrt{p_{10}} \pm \xi_{\varphi}\right)^{-1 / 2} \cos \theta, \varphi+G,-2^{1 / 2}\left(\sqrt{p_{10}} \pm \xi_{\varphi}\right)^{-1 / 2} \sin \theta \xi_{\theta}, \xi_{\varphi}\right)
\end{aligned}
$$

here $(x, y, \xi, \eta)$ are coordinates on $T^{*} \mathcal{M}$, with $\mathcal{M}=\mathbb{R}_{x} \times \mathbb{S}_{y}^{1}$ the model space. The function $G: T^{*} \mathbb{S}^{2} \rightarrow \mathbb{S}^{1}$ here is given by

$$
\left(\sqrt{p_{10}} \pm \xi_{\varphi}\right) \cos G=p_{10}^{1 / 2} \sin \theta \pm \frac{\xi_{\varphi}}{\sin \theta},\left(\sqrt{p_{10}} \pm \xi_{\varphi}\right) \sin G=\mp \cos \theta \xi_{\theta} .
$$

In fact, the maps $\Phi_{ \pm}$defined in (3.1) extend smoothly to the poles $\{\sin \theta=0\}$ of the sphere and satisfy the conditions of Proposition 3.5 on the complement of the opposite equator $\left\{\theta=\pi / 2, \xi_{\theta}=0, \xi_{\varphi}=\mp \sqrt{\tilde{\lambda}}\right\}$.

One can then conjugate the operators $P_{1}, P_{2}$ to some model operators $Q_{1}, Q_{2}$ as in Proposition 3.6. To bring the subprincipal terms in $Q_{j}$ to normal form, one still needs Moser averaging. Once the normal form of Proposition 3.6 is obtained, it is possible to use the ellipticity of $\mathbf{p}-(\tilde{\lambda}, \tilde{k})$ away from $\mathbf{p}^{-1}(\tilde{\lambda}, \tilde{k})$ (as in Proposition A.4) and spectral theory to obtain the quantization condition. The Grushin problem construction of Sect. 3.4 and Appendix A.1 is not needed, as the operator $P_{1}$ is self-adjoint.

\subsection{Hamiltonian Flow}

Let $(\theta, \varphi)$ be the spherical coordinates on $\mathbb{S}^{2}$ and let $\left(\xi_{\theta}, \xi_{\varphi}\right)$ be the corresponding momenta. Note that $\xi_{\theta}$ is defined away from the poles $\{\sin \theta=0\}$, while $\xi_{\varphi}$ is well defined and smooth on the whole $T^{*} \mathbb{S}^{2}$. In the $\left(\theta, \varphi, \xi_{\theta}, \xi_{\varphi}\right)$ coordinates, the principal symbols of $P_{2}$ and $P_{1}$ are $p_{20}=\xi_{\varphi}$ and

$$
p_{10}\left(\theta, \varphi, \xi_{\theta}, \xi_{\varphi}\right)=\Delta_{\theta} \xi_{\theta}^{2}+\frac{(1+\alpha)^{2}}{\Delta_{\theta} \sin ^{2} \theta}\left(\xi_{\varphi}-a \tilde{\omega} \sin ^{2} \theta\right)^{2}
$$

Since $p_{10}$ does not depend on $\varphi$, we have

$$
\left\{p_{10}, p_{20}\right\}=0 \text {. }
$$


We would like to apply the results of Sect. 2.4 on integrable Hamiltonian systems to establish a normal form for $\mathbf{p}=\left(p_{10}, p_{20}\right)$. First of all, we study the points where the integrable system $\mathbf{p}$ is degenerate:

Proposition 3.3. For a small enough,

1. For $C_{\theta}^{-1} \leq \tilde{\lambda} \leq C_{\theta}$, the set $\mathbf{p}^{-1}(\tilde{\lambda}, \tilde{k})$ is nonempty if and only if $(\tilde{\lambda}, \tilde{k}) \in \tilde{K}$.

2. The integrable system $\mathbf{p}$ is nondegenerate on $\mathbf{p}^{-1}(\widetilde{K})$, except at the equators

$$
\begin{aligned}
E_{ \pm}(\tilde{\lambda})= & \left\{\theta=\pi / 2, \xi_{\theta}=0,(1+\alpha)\left(\xi_{\varphi}-a \tilde{\omega}\right)= \pm \sqrt{\tilde{\lambda}}\right\} \subset T^{*} \mathbb{S}^{2}, \\
& C_{\theta}^{-1} \leq \tilde{\lambda} \leq C_{\theta} .
\end{aligned}
$$

Moreover, $p_{10}=\tilde{\lambda}$ on $E_{ \pm}(\tilde{\lambda})$ and the union of all $E_{ \pm}(\tilde{\lambda})$ is equal to $\mathbf{p}^{-1}\left(\tilde{K}_{ \pm}\right)$. Also,

$$
d p_{10}= \pm 2(1+\alpha) \sqrt{\tilde{\lambda}} d p_{20} \text { on } E_{ \pm}(\tilde{\lambda}) .
$$

Proof. We can verify directly the statements above for $a=0$, and also (3.2) for all $a$. Then, part 2 follows for small $a$ by a perturbation argument; part 1 follows from part 2 by studying the extremum problem for $\xi_{\varphi}$ restricted to $\left\{p_{10}=\tilde{\lambda}\right\}$.

Next, we construct the principal parts $F_{ \pm}$of the quantization symbols globally:

Proposition 3.4. For a small enough,

1. There exist unique smooth real-valued functions $F_{ \pm}(\tilde{\lambda}, \tilde{k})$ on $\widetilde{K}$ such that $\left.F_{ \pm}\right|_{\widetilde{K}_{ \pm}}=0$ and $\left(F_{ \pm}(\mathbf{p}), p_{20}\right)$ is a nondegenerate completely integrable system on $\mathbf{p}^{-1}\left(\widetilde{K} \backslash\left(\widetilde{K}_{+} \cup \widetilde{K}_{-}\right)\right)$with periodicity lattice $2 \pi \mathbb{Z}^{2}$.

2. $\partial_{\tilde{\lambda}} F_{ \pm}>0, \mp \partial_{\tilde{k}} F_{ \pm}>0$, and $F_{-}(\tilde{\lambda}, \tilde{k})-F_{+}(\tilde{\lambda}, \tilde{k})=2 \tilde{k}$ on $\tilde{K}$. In particular, one can define the inverse $F_{ \pm}^{-1}(\zeta, \tilde{k})$ of $F_{ \pm}$in the $\tilde{\lambda}$ variable, with $\tilde{k}$ as a parameter. Also, $F_{ \pm}=\sqrt{\tilde{\lambda}} \mp \tilde{k}$ for $a=0$ and $\partial_{\tilde{\lambda}} F_{ \pm}= \pm(2 \tilde{k})^{-1}+O\left(a^{2}\right)$ on $\widetilde{K}_{ \pm}$.

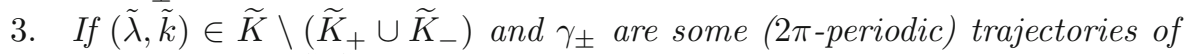
$F_{ \pm}(\mathbf{p})$ on $\mathbf{p}^{-1}(\tilde{\lambda}, \tilde{k})$, then

$$
\int_{\gamma_{ \pm}} \sigma^{S}=2 \pi F_{ \pm}(\tilde{\lambda}, \tilde{k}) .
$$

Proof. 1. We first construct $F_{+}$in a neighborhood of $\widetilde{K}_{+} \cdot \varepsilon_{k}>0$ and define $F_{+}$on the set

$$
\widetilde{K}_{\varepsilon}=\left\{(\tilde{\lambda}, \tilde{k}) \mid \tilde{k} \geq \varepsilon_{k},(1+\alpha)^{2}(\tilde{k}-a \tilde{\omega})^{2} \leq \tilde{\lambda} \leq C_{\theta}\right\} .
$$

We will pick $\varepsilon_{k}$ small enough so that $\widetilde{K}_{+} \subset \widetilde{K}_{\varepsilon}$; note, however, that $\widetilde{K}_{\varepsilon}$ does not lie in $\widetilde{K}$. Moreover, we will construct a symplectomorphism $\Phi$ from $\mathbf{p}^{-1}\left(\widetilde{K}_{\varepsilon}\right)$ onto a subset of $T^{*} \mathcal{M}$ such that $F_{+}(\mathbf{p}) \circ \Phi^{-1}=\zeta$ and $p_{20} \circ \Phi^{-1}=\eta$. 
Note that $\xi_{\varphi}=0$ on the poles of the sphere $\{\sin \theta=0\}$; therefore, $\left(\theta, \varphi, \xi_{\theta}, \xi_{\varphi}\right)$ is a symplectic system of coordinates near $\mathbf{p}^{-1}\left(\widetilde{K}_{\varepsilon}\right)$. Next, fix $\xi_{\varphi} \geq \varepsilon_{k}$ and consider $p_{10}$ as a function of $\left(\theta, \xi_{\theta}\right)$; then, for $a$ small enough, this function has a unique critical point $(0,0)$ on the compact set $\left\{p_{10}\left(\cdot, \cdot, \xi_{\varphi}\right) \leq C_{\theta}\right\}$; the Hessian at this point is positive definite. Indeed, it is enough to verify these statements for $a=0$ and check that $\partial_{\theta} p_{10}=\partial_{\xi_{\theta}} p_{10}=0$ for $\left(\theta, \xi_{\theta}\right)=(\pi / 2,0)$ and small $a$. Now, we may apply Proposition 2.10 to the function $\left\{p_{10}\left(\cdot, \cdot, \xi_{\varphi}\right)\right\}$ and obtain a function $F_{+}(\tilde{\lambda} ; \tilde{k})$ on $\widetilde{K}_{\varepsilon}$ such that $\left.F_{+}\right|_{\widetilde{K}_{+}}=0$ and $\partial_{\tilde{\lambda}} F_{+}>0$ and a mapping

$$
\Psi:\left(\theta, \xi_{\theta}, \xi_{\varphi}\right) \mapsto\left(\Psi_{x}\left(\theta, \xi_{\theta}, \xi_{\varphi}\right), \Psi_{\xi}\left(\theta, \xi_{\theta}, \xi_{\varphi}\right)\right)
$$

that defines a family of symplectomorphisms $\left(\theta, \xi_{\theta}\right) \mapsto\left(\Psi_{x}, \Psi_{\xi}\right)$, depending smoothly on the parameter $\xi_{\varphi}$, and

$$
\begin{aligned}
& F_{+}\left(p_{10}\left(\theta, \xi_{\theta}, \xi_{\varphi}\right), \xi_{\varphi}\right)=\frac{1}{2}\left(\Psi_{x}\left(\theta, \xi_{\theta}, \xi_{\varphi}\right)^{2}+\Psi_{\xi}\left(\theta, \xi_{\theta}, \xi_{\varphi}\right)^{2}\right), \\
& \left(\theta, \xi_{\theta}, \xi_{\varphi}\right) \in \mathbf{p}^{-1}\left(\widetilde{K}_{\varepsilon}\right) .
\end{aligned}
$$

Now, define $\Phi:\left(\theta, \varphi, \xi_{\theta}, \xi_{\varphi}\right) \mapsto\left(\Phi_{x}, \Phi_{y}, \Phi_{\xi}, \Phi_{\eta}\right) \in T^{*} \mathcal{M}$ by

$$
\Phi_{x}=\Psi_{x}, \Phi_{y}=\varphi+G\left(\Psi_{x}, \Psi_{\xi}, \xi_{\varphi}\right), \Phi_{\xi}=\Psi_{\xi}, \Phi_{\eta}=\xi_{\varphi} .
$$

Here $G\left(x, \xi ; \xi_{\varphi}\right)$ is some smooth function. For $\Phi$ to be a symplectomorphism, $G$ should satisfy

$$
\partial_{\xi} G\left(\Psi_{x}, \Psi_{\xi}, \xi_{\varphi}\right)=\partial_{\xi_{\varphi}} \Psi_{x}, \partial_{x} G\left(\Psi_{x}, \Psi_{\xi}, \xi_{\varphi}\right)=-\partial_{\xi_{\varphi}} \Psi_{\xi}
$$

Since $(x, \xi)$ vary in a disk, this system has a solution if and only if

$$
0=\left\{\Psi_{\xi}, \partial_{\xi_{\varphi}} \Psi_{x}\right\}+\left\{\partial_{\xi_{\varphi}} \Psi_{\xi}, \Psi_{x}\right\}=\partial_{\xi_{\varphi}}\left\{\Psi_{\xi}, \Psi_{x}\right\}
$$

this is true since $\left\{\Psi_{\xi}, \Psi_{x}\right\}=1$. The defining properties of $F_{+}$now follow from the corresponding properties of the integrable system $(\zeta, \eta)$; uniqueness follows from part 3 of Proposition 2.8 and the condition $\left.F_{+}\right|_{\widetilde{K}_{+}}=0$.

Now, by part 2 of Proposition 2.8 and Proposition 3.3, for each $\left(\tilde{\lambda}_{0}, \tilde{k}_{0}\right) \in \widetilde{K} \backslash\left(\widetilde{K}_{+} \cup \widetilde{K}_{-}\right)$, there exists a smooth function $F(\tilde{\lambda}, \tilde{k})$ defined in a neighborhood of $\left(\tilde{\lambda}_{0}, \tilde{k}_{0}\right)$ such that $\partial_{\lambda} F \neq 0$ and $\left(F(\mathbf{p}), p_{20}\right)$ has periodicity lattice $2 \pi \mathbb{Z}^{2}$; moreover, part 3 of Proposition 2.8 describes all possible $F$. Then, we can cover $\widetilde{K} \backslash \widetilde{K}_{\varepsilon}$ by a finite set of the neighborhoods above and modify the resulting functions $F$ and piece them together, to uniquely extend the function $F_{+}$constructed above from $\widetilde{K}_{\varepsilon}$ to $\widetilde{K} \backslash \widetilde{K}_{-}$. (Here we use that $\widetilde{K} \backslash \widetilde{K}_{-}$is simply connected). Similarly, we construct $F_{-}$on $\widetilde{K} \backslash \widetilde{K}_{+}$. (The fact that $F_{ \pm}$is smooth at $\widetilde{K}_{\mp}$ will follow from smoothness of $F_{\mp}$ at $\widetilde{K}_{\mp}$ and the identity $\left.F_{-}-F_{+}=2 \tilde{k}\right)$.

2. We can verify the formulas for $F_{ \pm}$for $a=0$ explicitly, using the fact that the Hamiltonian flow of $\sqrt{\tilde{\lambda}}$ is $2 \pi$-periodic in this case. The first two identities now follow immediately. As for the third one, we know by part 3 of Proposition 2.8 and the case $a=0$ that $F_{-}-F_{+}=2 \tilde{k}+c$ for some constant $c$; we can then show that $c=0$ using part 3 of this proposition. Finally, $\left.\partial_{\tilde{\lambda}} F_{ \pm}\right|_{\widetilde{K}_{ \pm}}$can be computed using Proposition 2.10. 
3. First, assume that $(\tilde{\lambda}, \tilde{k}) \in \tilde{K}_{\varepsilon}$ and let $\Phi$ be the symplectomorphism constructed in part 1 . Then

$$
\Phi \circ \gamma_{+}=\left\{\zeta=F_{+}(\tilde{\lambda}, \tilde{k}), \eta=\tilde{k}, \varphi=\mathrm{const}\right\}
$$

is a circle. Let $D_{+}$be the preimage under $\Phi$ of the disk with boundary $\Phi \circ \gamma_{+}$; then

$$
\int_{\gamma_{+}} \sigma^{S}=\int_{D_{+}} \omega^{S}=\int_{\Phi \circ D_{+}} \omega_{\mathcal{M}}^{S}=2 \pi F_{+}(\tilde{\lambda}, \tilde{k}) .
$$

We see that $(3.3)$ holds for $F_{+}$near $\widetilde{K}_{+}$; similarly, it holds for $F_{-}$ near $\widetilde{K}_{-}$. It now suffices to show that for each $\left(\tilde{\lambda}_{0}, \tilde{k}_{0}\right) \in \tilde{K} \backslash\left(\widetilde{K}_{+} \cup \widetilde{K}_{-}\right)$, there exists a neighborhood $V\left(\tilde{\lambda}_{0}, \tilde{k}_{0}\right)$ such that if $\left(\tilde{\lambda}_{j}, \tilde{k}_{j}\right) \in V, j=$

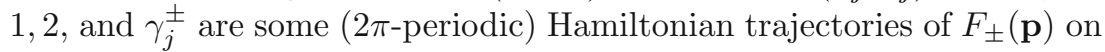
$\mathbf{p}^{-1}\left(\tilde{\lambda}_{j}, \tilde{k}_{j}\right)$, then

$$
\int_{\gamma_{2}^{ \pm}} \sigma^{S}-\int_{\gamma_{1}^{ \pm}} \sigma^{S}=2 \pi\left(F_{ \pm}\left(\tilde{\lambda}_{2}, \tilde{k}_{2}\right)-F_{ \pm}\left(\tilde{\lambda}_{1}, \tilde{k}_{1}\right)\right) .
$$

In particular, if (3.3) holds for one point of $V$, it holds on the whole $V$. One way to prove (3.4) is to use part 1 of Proposition 2.9 to conjugate $\left(F_{ \pm}(\mathbf{p}), p_{20}\right)$ to the system $\left(\xi_{x}, \xi_{y}\right)$ on the torus $\mathbb{T}_{x, y}$ and note that the left-hand side of (3.4) is the integral of the symplectic form over a certain submanifold bounded by $\gamma_{1}, \gamma_{2}$; therefore, it is the same for the conjugated system, where it can be computed explicitly.

Finally, we construct local symplectomorphisms conjugating $\left(F_{ \pm}(\mathbf{p})\right.$, $\left.p_{20}\right)$ to $(\zeta, \eta)$ :

Proposition 3.5. For each $\left(\tilde{\lambda}_{0}, \tilde{k}_{0}\right) \in \widetilde{K} \backslash \widetilde{K}_{\mp}$, there exists an exact symplectomorphism $\Phi_{ \pm}$from a neighborhood of $\mathbf{p}^{-1}\left(\tilde{\lambda}_{0}, \tilde{k}_{0}\right)$ in $T^{*} \mathbb{S}^{2}$ onto a neighborhood of

$$
\Lambda_{\mathcal{M}}=\left\{\zeta=F_{ \pm}\left(\tilde{\lambda}_{0}, \tilde{k}_{0}\right), \eta=\tilde{k}_{0}\right\}
$$

in $T^{*} \mathcal{M}$ such that

$$
p_{10} \circ \Phi_{ \pm}^{-1}=F_{ \pm}^{-1}(\zeta, \eta), p_{20} \circ \Phi_{ \pm}^{-1}=\eta .
$$

Proof. The existence of $\Phi_{ \pm}$away from $\widetilde{K}_{ \pm}$follows from part 1 of Proposition 2.9 , applied to the systems $\left(F_{ \pm}(\mathbf{p}), p_{20}\right)$ and $(\zeta, \eta)$; near $\widetilde{K}_{ \pm}$, these symplectomorphisms have been constructed in the proof of part 1 of Proposition 3.4. Exactness follows by part 2 of Proposition 2.9 (which still applies in the degenerate case); the equality of classical actions over the flows of $F_{ \pm}(\mathbf{p})$ and $\zeta$ follows from part 3 of Proposition 3.4, while the classical actions over the flows of both $p_{20}$ on $\mathbf{p}^{-1}\left(\tilde{\lambda}_{0}, \tilde{k}_{0}\right)$ and $\eta$ on $\Lambda_{\mathcal{M}}$ are both equal to $2 \pi \tilde{k}_{0}$. 


\subsection{Moser Averaging}

Fix $\left(\tilde{\lambda}_{0}, \tilde{k}_{0}\right) \in \widetilde{K} \backslash \widetilde{K}_{\mp}$, take small $\varepsilon>0$, and define (suppressing the dependence on the choice of the sign)

$$
\begin{aligned}
& \Lambda^{0}=\mathbf{p}^{-1}\left(\tilde{\lambda}_{0}, \tilde{k}_{0}\right), \zeta_{0}=F_{ \pm}\left(\tilde{\lambda}_{0}, \tilde{k}_{0}\right), \Lambda_{\mathcal{M}}^{0}=\left\{\zeta=\zeta_{0}, \eta=\tilde{k}_{0}\right\} \subset T^{*} \mathcal{M}, \\
& V^{\varepsilon}=\left\{(\tilde{\lambda}, \tilde{k})|| F_{ \pm}(\tilde{\lambda}, \tilde{k})-\zeta_{0}|\leq \varepsilon,| \tilde{k}-\tilde{k}_{0} \mid \leq \varepsilon\right\} \subset \mathbb{R}^{2} \\
& V_{\mathcal{M}}^{\varepsilon}=\left\{\left|\zeta-\zeta_{0}\right| \leq \varepsilon,\left|\eta-\tilde{k}_{0}\right| \leq \varepsilon\right\} \subset T^{*} \mathcal{M}
\end{aligned}
$$

then $V^{\varepsilon}$ and $V_{\mathcal{M}}^{\varepsilon}$ are compact neighborhoods of $\left(\tilde{\lambda}_{0}, \tilde{k}_{0}\right)$ and $\Lambda_{\mathcal{M}}^{0}$, respectively. Here the functions $F_{ \pm}$are as in Proposition 3.4. Let $\Phi_{ \pm}$be the symplectomorphism constructed in Proposition 3.5; we know that for $\varepsilon$ small enough, $\Phi_{ \pm}\left(\mathbf{p}^{-1}\left(V^{\varepsilon}\right)\right)=V_{\mathcal{M}}^{\varepsilon}$. In this subsection, we prove

Proposition 3.6. For $\left(\tilde{\lambda}_{0}, \tilde{k}_{0}\right) \in \widetilde{K} \backslash \widetilde{K}_{ \pm}$and $\varepsilon>0$ small enough, there exists a pair of operators $\left(B_{1}, B_{2}\right)$ quantizing $\Phi_{ \pm}$near $\mathbf{p}^{-1}\left(V^{\varepsilon}\right) \times V_{\mathcal{M}}^{\varepsilon}$ in the sense of Proposition 2.5 and operators $Q_{1}, Q_{2} \in \Psi_{\mathrm{cl}}^{\mathrm{comp}}(\mathcal{M})$ such that:

1. $P_{1}$ and $P_{2}$ are intertwined with $Q_{1}$ and $Q_{2}$, respectively, via $\left(B_{1}, B_{2}\right)$, near $\mathbf{p}^{-1}\left(V^{\varepsilon}\right) \times V_{\mathcal{M}}^{\varepsilon}$. It follows immediately that the principal symbols of $Q_{1}$ and $Q_{2}$ are $F_{ \pm}^{-1}(\zeta, \eta)$ and $\eta$, respectively, near $V_{\mathcal{M}}^{\varepsilon}$.

2. $Q_{2}=h D_{y}$ and the full symbol of $Q_{1}$ is a function of $(\zeta, \eta)$, microlocally near $V_{\mathcal{M}}^{\varepsilon}$. Here we use Weyl quantization on $\mathcal{M}$, inherited from the covering space $\mathbb{R}^{2}$.

First of all, we use Proposition 2.5 to find some $\left(B_{1}, B_{2}\right)$ quantizing $\Phi_{ \pm}$ and $Q_{1}, Q_{2}$ intertwined with $P_{1}$ and $P_{2}$ by $\left(B_{1}, B_{2}\right)$. Then, we will find a couple of operators $X, Y \in \Psi_{\mathrm{cl}}^{\mathrm{comp}}(\mathcal{M})$ such that $Y=X^{-1}$ near $V_{\mathcal{M}}^{\varepsilon}$ and the operators $Q_{1}^{\prime}=X Q_{1} Y, Q_{2}^{\prime}=X Q_{2} Y$ satisfy part 2 of Proposition 3.6. This is the content of this subsection and will be done in several conjugations by pseudodifferential operators using Moser averaging technique. We can then change $B_{1}, B_{2}$ following the remark after Proposition 2.5 so that $P_{1}$ and $P_{2}$ are intertwined with $Q_{1}^{\prime}$ and $Q_{2}^{\prime}$, which finishes the proof.

The averaging construction is based on the following

Proposition 3.7. Assume that the functions $p_{0}, f_{0}, g \in C^{\infty}\left(V_{\mathcal{M}}^{\varepsilon}\right)$ are given by one of the following:

1. $p_{0}=f_{0}=\eta$ and $g$ is arbitrary;

2. $p_{0}=\zeta$ and $f_{0}=f_{0}(\zeta, \eta)$ is smooth in $V_{\mathcal{M}}^{\varepsilon}$, with $\partial_{\zeta} f_{0} \neq 0$ everywhere, and $g$ is independent of $y$.

Define

$$
\langle g\rangle=\frac{1}{2 \pi} \int_{0}^{2 \pi} g \circ \exp \left(t H_{p_{0}}\right) \mathrm{d} t .
$$

Then, there exists unique $b \in C^{\infty}\left(V_{\mathcal{M}}^{\varepsilon}\right)$ such that $\langle b\rangle=0$ and

$$
g=\langle g\rangle+\left\{f_{0}, b\right\} .
$$

Moreover, in case (2) $b$ is independent of $y$. 
Proof. We only consider case (2); case (1) is proven in a similar fashion. First of all, if $b$ is $y$-independent, then $\left\{f_{0}, b\right\}=\partial_{\zeta} f_{0} \cdot\{\zeta, b\}=\left\{\zeta, \partial_{\zeta} f_{0} \cdot b\right\}$; therefore, without loss of generality we may assume that $f_{0}=\zeta$. The existence and uniqueness of $b$ now follows immediately if we treat $y, \eta$ as parameters and consider polar coordinates in the $(x, \xi)$ variables. To show that $b$ is smooth at $\zeta=0$ (in case $\zeta_{0} \leq \varepsilon$ ), let $z=x+i \xi$ and decompose $g-\langle g\rangle$ into an asymptotic sum of the terms $z^{j} \bar{z}^{k}$ with $j, k \geq 0, j \neq k$, and coefficients smooth in $(y, \eta)$; the term in $b$ corresponding to $z^{j} \bar{z}^{k}$ is $z^{j} \bar{z}^{k} /(i(k-j))$.

Henceforth in this subsection we will work with the operators $Q_{j}$ on the level of their full symbols, microlocally in a neighborhood of $V_{\mathcal{M}}^{\varepsilon}$. (The operators $X$ and $Y$ will then be given by the product of all operators used in conjugations below, multiplied by an appropriate cutoff). Denote by $q_{j}$ the full symbol of $Q_{j}$. We argue in three steps, following in part [31, Section 3].

Step 1: Use Moser averaging to make $q_{2}$ independent of $y$.

Assume that $q_{2}$ is independent of $y$ modulo $O\left(h^{n+1}\right)$ for some $n \geq 0$; more precisely,

$$
q_{2}=\sum_{j=0}^{n} h^{j} q_{2, j}(x, \xi, \eta)+h^{n+1} r_{n}(x, y, \xi, \eta)+O\left(h^{n+2}\right) .
$$

Take some $B \in \Psi_{\mathrm{cl}}^{\text {loc }}$ with principal symbol $b$ and consider the conjugated operator

$$
Q_{2}^{\prime}=\exp \left(i h^{n} B\right) Q_{2} \exp \left(-i h^{n} B\right) .
$$

Here $\exp \left( \pm i h^{n} B\right) \in \Psi_{\mathrm{cl}}^{\text {loc }}$ are well defined by Proposition 2.7 and inverse to each other; using the same proposition, we see that the full symbol of $Q_{2}^{\prime}$ is

$$
\sum_{j=0}^{n} h^{j} q_{2, j}(x, \xi, \eta)+h^{n+1}\left(r_{n}-\{\eta, b\}\right)+O\left(h^{n+2}\right) .
$$

If we choose $b$ as in Proposition 3.7(1), then $r_{n}-\{\eta, b\}=\left\langle r_{n}\right\rangle$ is a function of $(x, \xi, \eta)$ only; thus, the full symbol of $Q_{2}^{\prime}$ is independent of $y$ modulo $O\left(h^{n+2}\right)$. Arguing by induction and taking the asymptotic product of the resulting sequence of exponentials, we make the full symbol of $Q_{2}$ independent of $y$.

Step 2: Use our knowledge of the spectrum of $P_{2}$ to make $q_{2}=\eta$.

First of all, we claim that

$$
\exp \left(2 \pi i Q_{2} / h\right)=I
$$

microlocally near $V_{\mathcal{M}}^{\varepsilon} \times V_{\mathcal{M}}^{\varepsilon}$. For that, we will use Proposition 2.6. Let $X \in \Psi_{\mathrm{cl}}^{\mathrm{comp}}\left(\mathbb{S}^{2}\right)$ have real-valued principal symbol, be microlocalized in a small neighborhood of $\mathbf{p}^{-1}\left(V^{\varepsilon}\right)$, but equal to the identity microlocally near this set. Consider

$$
\mathcal{P}_{t}=\exp \left(i t Q_{2} / h\right) B_{1} \exp \left(-i t\left(P_{2} X\right) / h\right) B_{2} .
$$


We see that

$$
h D_{t} \mathcal{P}_{t}=\exp \left(i t Q_{2} / h\right)\left(Q_{2} B_{1}-B_{1} P_{2} X\right) \exp \left(-i t\left(P_{2} X\right) / h\right) B_{2}
$$

vanishes microlocally near $V_{\mathcal{M}}^{\varepsilon} \times V_{\mathcal{M}}^{\varepsilon}$; integrating between 0 and $2 \pi$ and using part 5 of Proposition 2.6 to show that $\exp \left(-2 \pi i\left(P_{2} X\right) / h\right)=I$ microlocally near $\mathbf{p}^{-1}\left(V^{\varepsilon}\right) \times \mathbf{p}^{-1}\left(V^{\varepsilon}\right)$, we get $(3.5)$.

Now, let $X_{\mathcal{M}} \in \Psi_{\mathrm{cl}}^{\mathrm{comp}}(\mathcal{M})$ be equal to the identity microlocally near $\mathrm{WF}_{h}\left(Q_{2}\right)$; since the full symbol of $Q_{2}$ is independent of $y$, we have $\left[Q_{2},\left(h D_{y}\right) X_{\mathcal{M}}\right]=O\left(h^{\infty}\right)$. Therefore, by parts 2 and 5 of Proposition 2.6

$$
\exp \left(2 \pi i\left(Q_{2}-\left(h D_{y}\right) X_{\mathcal{M}}\right) / h\right)=\exp \left(-2 \pi i D_{y} X_{\mathcal{M}}\right) \exp \left(2 \pi i Q_{2} / h\right)=I
$$

microlocally near $V_{\mathcal{M}}^{\varepsilon} \times V_{\mathcal{M}}^{\varepsilon}$. However, $R=h^{-1}\left(Q_{2}-\left(h D_{y}\right) X_{\mathcal{M}}\right) \in \Psi_{\mathrm{cl}}^{\text {loc }}$ near $V_{\mathcal{M}}^{\varepsilon}$ and thus the left-hand side $\exp (2 \pi i R)$ is pseudodifferential; by part 3 of Proposition 2.7, we get $R=l$ for some constant $l \in \mathbb{Z}$ and therefore

$$
Q_{2}=h D_{y}+h l
$$

microlocally near $V_{\mathcal{M}}^{\varepsilon}$. It remains to conjugate $Q_{2}$ by $e^{i l y}$ to get $q_{2}=\eta$.

Step 3: Use Moser averaging again to make $q_{1}$ a function of $(\zeta, \eta)$, while preserving $q_{2}=\eta$.

Recall that $\left[P_{1}, P_{2}\right]=0$; therefore, $\left[Q_{1}, Q_{2}\right]=0$ (microlocally near $\left.V_{\mathcal{M}}^{\varepsilon}\right)$. Since $q_{2}=\eta$, this means that $q_{1}$ is independent of $y$. We now repeat the argument of Step 1, using Proposition 3.7(2) with $f_{0}=F_{ \pm}^{-1}(\zeta, \eta)$. The function $b$ at each step is independent of $y$; thus, we can take $\left[B, h D_{y}\right]=O\left(h^{\infty}\right)$. But in that case, conjugation by $\exp \left(i h^{n} B\right)$ does not change $Q_{2}$; the symbol of the conjugated $Q_{1}$ is still independent of $y$. Finally, $\left\langle r_{n}\right\rangle$ is a function of $(\zeta, \eta)$; therefore, $q_{1}$ after conjugation will also be a function of $(\zeta, \eta)$.

\subsection{Construction of the Grushin Problem}

In this subsection, we establish a local quantization condition:

Proposition 3.8. 1. Assume that $\left(\tilde{\lambda}_{0}, \tilde{k}_{0}\right) \in \widetilde{K} \backslash \widetilde{K}_{\mp}$ and $V_{\varepsilon}$ is the neighborhood of $\left(\tilde{\lambda}_{0}, \tilde{k}_{0}\right)$ introduced in the beginning of Sect. 3.3. Then, for $\varepsilon>0$ small enough, there exists a classical symbol $\widetilde{G}_{ \pm}(\tilde{\lambda}, \tilde{k} ; h)$ on $V_{\varepsilon}$ with principal symbol $F_{ \pm}$and such that for $k \in \mathbb{Z}$, the poles $\tilde{\lambda}+i h \tilde{\mu}$ of $R_{\theta}(\omega, \lambda, k)$ with $(\tilde{\lambda}, \tilde{k}) \in V_{\varepsilon}$ and $|\tilde{\mu}| \leq C_{\theta}$ are simple with polynomial resolvent estimate $L^{2} \rightarrow L^{2}$, in the sense of Definition 1.1, and coincide modulo $O\left(h^{\infty}\right)$ with the solution set of the quantization condition

$$
\widetilde{G}_{ \pm}(\tilde{\lambda}+i h \tilde{\mu}, \tilde{k} ; h) \in h \mathbb{N} .
$$

2. Assume that $\left(\tilde{\lambda}_{0}, \tilde{k}_{0}\right)$ satisfies (1.15), but does not lie in $\widetilde{K}$. Then, there exists a neighborhood $V\left(\tilde{\lambda}_{0}, \tilde{k}_{0}\right)$ such that for $h$ small enough, there are no elements $(\tilde{\lambda}+i h \tilde{\mu}, \tilde{k})$ of the joint spectrum of $P_{1}, P_{2}$ with $(\tilde{\lambda}, \tilde{k}) \in V$ and $|\tilde{\mu}| \leq C_{\theta}$, and $R_{\theta}(\omega, \lambda, k)$ is bounded $L^{2} \rightarrow L^{2}$ by $O\left(h^{2}\right)$. 
To prove part 1 , we will use the microlocal conjugation constructed above. Let $\left(\tilde{\lambda}_{0}, \tilde{k}_{0}\right) \in \widetilde{K} \backslash \widetilde{K}_{\mp}$ and $\varepsilon>0, B_{1}, B_{2}, Q_{1}, Q_{2}$ be given by Proposition 3.6. Consider the operators

$$
T_{1}=\frac{1}{2}\left(\left(h D_{x}\right)^{2}+x^{2}\right)-\frac{h}{2}, \quad T_{2}=h D_{y}
$$

on $\mathcal{M}$; their full symbols are $\zeta-h / 2$ and $\eta$, respectively. We know that $T_{1}$ and $T_{2}$ commute; the joint spectrum of $T_{1}, T_{2}$ is $h(\mathbb{N} \times \mathbb{Z})$. Therefore, for any bounded function $f$ on $\mathbb{R}^{2}$, we can define $f\left(T_{1}, T_{2}\right)$ by means of spectral theory; this is a bounded operator on $L^{2}(\mathcal{M})$.

Proposition 3.9. 1. For $f \in C_{0}^{\infty}\left(\mathbb{R}^{2}\right)$, the operator $f\left(T_{1}, T_{2}\right)$ is pseudodifferential; moreover, $f\left(T_{1}, T_{2}\right) \in \Psi_{\mathrm{cl}}^{\mathrm{comp}}(\mathcal{M})$ and $\mathrm{WF}_{h}\left(f\left(T_{1}, T_{2}\right)\right) \subset\{(\zeta, \eta) \in$ $\operatorname{supp} f\}$. The full symbol of $f\left(T_{1}, T_{2}\right)$ in the Weyl quantization is a function of $\zeta$ and $\eta$ only; the principal symbol is $f(\zeta, \eta)$.

2. Assume that $\zeta_{1} \in h \mathbb{N}, \eta_{1} \in h \mathbb{Z}$. Let $u$ be the $L^{2}$ normalized joint eigenfunction of $\left(T_{1}, T_{2}\right)$ with eigenvalue $\left(\zeta_{1}, \eta_{1}\right)$. Then, $u$ is compactly microlocalized and

$$
\mathrm{WF}_{h}(u) \subset\left\{\zeta=\zeta_{1}, \eta=\eta_{1}\right\}
$$

3. Assume that the function $f(\zeta, \eta ; h)$ is Borel measurable has support contained in a compact $h$-independent subset $K_{f}$ of $\mathbb{R}^{2}$, and

$$
\max \{|f(\zeta, \eta ; h)| \mid \zeta \in h \mathbb{N}, \eta \in h \mathbb{Z}\} \leq C h^{-r}
$$

for some $r \geq 0$. Then, the operator $f\left(T_{1}, T_{2} ; h\right)$ is compactly microlocalized, its wavefront set is contained in the square of $\left\{(\zeta, \eta) \in K_{f}\right\}$, and the operator norm of $f\left(T_{1}, T_{2} ; h\right)$ is $O\left(h^{-r}\right)$.

Proof. For part 1, we can show that the operator $f\left(T_{1}, T_{2}\right)$ is pseudodifferential by means of Helffer-Sjöstrand formula in calculus of several commuting pseudodifferential operators; see for example [21, Chapter 8]. This also gives information on the principal symbol and the wavefront set of this operator. To show that the full symbol of $f\left(T_{1}, T_{2}\right)$ depends only on $(\zeta, \eta)$, note that if $A \in \Psi^{\text {loc }}(\mathcal{M})$ and $a$ is its full symbol in the Weyl quantization, then the full symbol of $\left[A, T_{1}\right]$ in the Weyl quantization is $-i h\{a, \zeta\}$; similarly, the full symbol of $\left[A, T_{2}\right]$ in the Weyl quantization is $-i h\{a, \eta\}$ (see for example [43, discussion before (1.11)]). Since $\left[f\left(T_{1}, T_{2}\right), T_{j}\right]=0$, the full symbol of $f\left(T_{1}, T_{2}\right)$ Poisson commutes with $\zeta$ and $\eta$.

To show part 2 , we take $\chi(\zeta, \eta) \in C_{0}^{\infty}\left(\mathbb{R}^{2}\right)$ equal to 1 near $\left(\zeta_{1}, \eta_{1}\right)$; then, $u=\chi\left(T_{1}, T_{2}\right) u$. Similarly, to show part 3 , we take $\chi$ equal to 1 near $K_{f}$; then, the $L^{2}$ operator norm of $f\left(T_{1}, T_{2}\right)$ can be estimated easily and $f\left(T_{1}, T_{2}\right)=$ $\chi\left(T_{1}, T_{2}\right) f\left(T_{1}, T_{2}\right) \chi\left(T_{1}, T_{2}\right)$.

Now, recall that by Proposition 3.6, the full symbol of $Q_{1}$ in the Weyl quantization is a function of $(\zeta, \eta)$ near $V_{\mathcal{M}}^{\varepsilon}$; therefore, we can find a compactly supported symbol $\widetilde{G}_{ \pm}(\tilde{\lambda}, \tilde{k} ; h)$ such that the principal symbol of $\widetilde{G}_{ \pm}$near $V^{\varepsilon}$ is $F_{ \pm}$and

$$
Q_{1}=\widetilde{G}_{ \pm}^{-1}\left(T_{1}, T_{2} ; h\right)
$$


microlocally near $V_{\mathcal{M}}^{\varepsilon}$, where $\widetilde{G}_{ \pm}^{-1}(\zeta, \tilde{k} ; h)$ is the inverse of $\widetilde{G}_{ \pm}$in the $\tilde{\lambda}$ variable. Recall also that $Q_{2}=T_{2}$ microlocally near $V_{\mathcal{M}}^{\varepsilon}$. Multiplying $Q_{1}, Q_{2}$ by an appropriate cutoff, which is a function of $T_{1}, T_{2}$, we can assume that $Q_{1}, Q_{2}$ are functions of $T_{1}, T_{2}$ modulo $h^{\infty} \Psi^{-\infty}$. We can now construct a local Grushin problem for $Q_{1}, Q_{2}$ :

Proposition 3.10. Let $\left(\tilde{\lambda}_{1}, \tilde{k}_{1}\right) \in V^{\varepsilon}$ and $\left|\tilde{\mu}_{1}\right| \leq C_{\theta}$.

1. Assume that $\left(\tilde{\lambda}_{1}+i h \tilde{\mu}_{1}, \tilde{k}_{1}\right)$ satisfies (3.6), with $\zeta_{1}=\widetilde{G}_{ \pm}\left(\tilde{\lambda}_{1}+i h \tilde{\mu}_{1}, \tilde{k}_{1}\right) \in$ $h \mathbb{N}$. Then, there exist operators $A_{1}, A_{2}, S_{1}, S_{2}$ such that conditions (L1)(L5) of Appendix A.2 are satisfied, with $r=1,\left(P_{1}, P_{2}\right)$ replaced by $\left(Q_{1}-\right.$ $\left.\tilde{\lambda}_{1}-i h \tilde{\mu}_{1}, Q_{2}-\tilde{k}_{1}\right), K=\left\{\zeta=\zeta_{1}, \eta=\tilde{k}_{1}\right\}$, and

$$
A_{1}\left(Q_{1}-\tilde{\lambda}_{1}-i h \tilde{\mu}_{1}\right)+A_{2}\left(Q_{2}-\tilde{k}_{1}\right)=I-S_{1} S_{2}
$$

microlocally near $V_{\mathcal{M}}^{\varepsilon} \times V_{\mathcal{M}}^{\varepsilon}$.

2. Fix $\delta>0$ and assume that

$$
\left|\left(\tilde{\lambda}_{1}+i h \tilde{\mu}_{1}, \tilde{k}_{1}\right)-\left(G_{ \pm}^{-1}(\zeta, \eta), \eta\right)\right| \geq \delta h, \zeta \in h \mathbb{N}, \eta \in h \mathbb{Z} .
$$

Then, there exist operators $A_{1}, A_{2}$ such that the conditions (L1)-(L2) of Appendix A.2 are satisfied, with $r=1,\left(P_{1}, P_{2}\right)$ replaced by $\left(Q_{1}-\tilde{\lambda}_{1}-\right.$ $\left.i h \tilde{\mu}_{1}, Q_{2}-\tilde{k}_{1}\right), K=\left\{\zeta=\zeta_{1}, \eta=\tilde{k}_{1}\right\}$, and

$$
A_{1}\left(Q_{1}-\tilde{\lambda}_{1}-i h \tilde{\mu}_{1}\right)+A_{2}\left(Q_{2}-\tilde{k}_{1}\right)=I
$$

microlocally near $V_{\mathcal{M}}^{\varepsilon} \times V_{\mathcal{M}}^{\varepsilon}$.

Proof. 1. Let $S_{1}: \mathbb{C} \rightarrow L^{2}(\mathcal{M})$ and $S_{2}: L^{2}(\mathcal{M}) \rightarrow \mathbb{C}$ be the inclusion and the orthogonal projection onto, respectively, the unit joint eigenfunction of $\left(T_{1}, T_{2}\right)$ with eigenvalue $\left(\zeta_{1}, \tilde{k}_{1}\right)$. The properties (L3) and (L4) now follow from part 2 of Proposition 3.9.

Next, we use a partition of unity on the circle to construct the functions $\chi_{1}, \chi_{2}$ with the following properties:

- $\chi_{j} \in C^{\infty}\left(\mathbb{R}^{2} \backslash 0\right)$ is positively homogeneous of degree 0 ;

- $\quad \chi_{j} \geq 0$ and $\chi_{1}+\chi_{2}=1$ everywhere on $\mathbb{R}^{2} \backslash 0$;

- $\chi_{j}\left(s_{1}, s_{2}\right)=0$ for $\left|s_{j}\right|<\left|s_{3-j}\right| / 2$.

It follows that

$$
\left|s_{j}^{-1} \chi_{j}\left(\left|s_{1}\right|^{2},\left|s_{2}\right|^{2}\right)\right| \leq C\left(\left|s_{1}\right|+\left|s_{2}\right|\right)^{-1} .
$$

Take $\chi(\zeta, \eta) \in C_{0}^{\infty}$ supported in a small neighborhood of $V_{\mathcal{M}}^{\varepsilon}$, while equal to 1 near $V_{\mathcal{M}}^{\varepsilon}$; define the functions $f_{1}, f_{2}$ as follows:

$$
\begin{aligned}
& f_{1}(\zeta, \eta ; h)=\frac{\chi(\zeta, \eta) \chi_{1}\left(\left|\widetilde{G}_{ \pm}^{-1}(\zeta, \eta ; h)-\tilde{\lambda}_{1}-i h \tilde{\mu}_{1}\right|^{2},\left|\eta-\tilde{k}_{1}\right|^{2}\right)}{\widetilde{G}_{ \pm}^{-1}(\zeta, \eta ; h)-\tilde{\lambda}_{1}-i h \tilde{\mu}_{1}} \\
& f_{2}(\zeta, \eta ; h)=\frac{\chi(\zeta, \eta) \chi_{2}\left(\left|\widetilde{G}_{ \pm}^{-1}(\zeta, \eta ; h)-\tilde{\lambda}_{1}-i h \tilde{\mu}_{1}\right|^{2},\left|\eta-\tilde{k}_{1}\right|^{2}\right)}{\eta-\tilde{k}_{1}}
\end{aligned}
$$

for $(\zeta, \eta) \neq\left(\zeta_{1}, \tilde{k}_{1}\right)$; we put $f_{j}\left(\zeta_{1}, \tilde{k}_{1}\right)=0$. We now take $A_{j}=$ $f_{j}\left(T_{1}, T_{2} ; h\right)$. Noticing that

$\left|\widetilde{G}_{ \pm}^{-1}(\zeta, \eta ; h)-\tilde{\lambda}_{1}\right|+\left|\eta-\tilde{k}_{1}\right| \geq h / C,(\zeta, \eta) \in h(\mathbb{N} \times \mathbb{Z}) \cap \operatorname{supp} \chi \backslash\left(\zeta_{1}, \tilde{k}_{1}\right)$, 
and using Proposition 3.9 and (3.8), we get that $A_{j}$ are compactly microlocalized and $\left\|A_{j}\right\|=O\left(h^{-1}\right)$. Moreover, if $\tilde{\chi}(\zeta, \eta)$ is equal to 1 near $\left(\zeta_{1}, \tilde{k}_{1}\right)$, then $(1-\tilde{\chi}) f_{j}$ are smooth symbols; then, $A_{j}^{\prime \prime}=(1-\tilde{\chi})\left(T_{1}, T_{2}\right) A_{j}$ belongs to $\Psi_{\mathrm{cl}}^{\text {comp }}$ by part 1 of Proposition 3.9 and $A_{j}^{\prime}=\tilde{\chi}\left(T_{1}, T_{2}\right) A_{j}$ is microlocalized in the Cartesian square of $\{(\zeta, \eta) \in \operatorname{supp} \tilde{\chi}\}$; we have established property (L1), with $r=1$. The properties (L2), (L5), and (3.7) are easy to verify, given that all the operators of interest are functions of $T_{1}, T_{2}$.

2. This is proved similarly to part 1 .

Finally, we conjugate the operators of the previous proposition by $B_{1}, B_{2}$ to get a local Grushin problem for $P_{1}, P_{2}$ and obtain information about the joint spectrum:

Proof of Propositon 3.8. 1. Assume first that $\tilde{\lambda}_{1}, \tilde{k}_{1}, \tilde{\mu}_{1}$ satisfy the conditions of part 1 of Proposition 3.10; let $A_{1}, A_{2}, S_{1}, S_{2}$ be the operators constructed there. Recall that $A_{j}$ are microlocalized in a small neighborhood of $V_{\mathcal{M}}^{\varepsilon}$. Then, the operators

$$
\widetilde{A}_{j}=B_{2} A_{j} B_{1}, \widetilde{S}_{1}=B_{2} S_{1}, \widetilde{S}_{2}=S_{2} B_{1},
$$

together with $P_{1}-\tilde{\lambda}_{1}-i h \tilde{\mu}_{1}, P_{2}-\tilde{k}_{1}$ in place of $P_{1}, P_{2}$ satisfy the conditions (L1)-(L5) of Appendix A.2 with $K=\mathbf{p}^{-1}\left(\tilde{\lambda}_{1}, \tilde{k}_{1}\right)$ and

$$
\widetilde{A}_{1}\left(P_{1}-\tilde{\lambda}_{1}-i h \tilde{\mu}_{1}\right)+\widetilde{A}_{2}\left(P_{2}-\tilde{k}_{1}\right)=I-\widetilde{S}_{1} \widetilde{S}_{2}
$$

microlocally near $\mathbf{p}^{-1}\left(V^{\varepsilon}\right)$. Moreover, $P_{1}-\tilde{\lambda}_{1}-i h \tilde{\mu}_{1}, P_{2}-\tilde{k}_{1}$ satisfy conditions (E1)-(E2) of Appendix A.2 and the set where both their principal symbols vanish is exactly $K$. We can now apply part 2 of Proposition A.4 to show that for $h$ small enough and some $\delta>0$, independent of $h, \tilde{\lambda}_{1}, \tilde{k}_{1}$, there is exactly one element of the joint spectrum of $\left(P_{1}, P_{2}\right)$ in the ball of radius $\delta h$ centered at $\left(\tilde{\lambda}_{1}+i h \tilde{\mu}_{1}, \tilde{k}_{1}\right)$, and this point is within $O\left(h^{\infty}\right)$ of the center of the ball.

Now, we assume that $(\tilde{\lambda}+i h \tilde{\mu}, \tilde{k})$ satisfies the conditions of part 2 of Proposition 3.10, with $\delta$ specified in the previous paragraph. Then, we can argue as above, using part 1 of Proposition A.4, to show that this point does not lie in the joint spectrum for $h$ small enough.

Since every point $(\tilde{\lambda}+i h \tilde{\mu}, \tilde{k})$ such that $(\tilde{\lambda}, \tilde{k}) \in V^{\varepsilon}$ and $|\tilde{\mu}| \leq C_{\theta}$ is covered by one of the two cases above, we have established that the angular poles in the indicated region coincide modulo $O\left(h^{\infty}\right)$ with the set of solutions to the quantization condition. Moreover, Proposition A.3 together with the construction of a global Grushin problem from a local one carried out in the proof of Proposition A.4 provides the resolvent estimates required in Definition 1.1.

2. The set $\mathbf{p}^{-1}\left(\tilde{\lambda}_{0}, \tilde{k}_{0}\right)$ is empty by Proposition 3.3 ; therefore, the operator

$$
T=\left(P_{1}-\tilde{\lambda}-i h \tilde{\mu}\right)^{*}\left(P_{1}-\tilde{\lambda}-i h \tilde{\mu}\right)+\left(P_{2}-\tilde{k}\right)^{2}
$$


is elliptic in the class $\Psi^{2}\left(\mathbb{S}^{2}\right)$ for $(\tilde{\lambda}, \tilde{k})$ close to $\left(\tilde{\lambda}_{0}, \tilde{k}_{0}\right)$ and $\tilde{\mu}$ bounded; therefore, for $h$ small enough, $\left\|T^{-1}\right\|_{L^{2} \rightarrow L^{2}}=O(1)$. The absence of joint spectrum and resolvent estimate follow immediately if we notice that the restriction of $T$ to $\mathcal{D}_{k}^{\prime}$ is $h^{4}\left(P_{\theta}-\lambda\right)^{*}\left(P_{\theta}-\lambda\right)$.

\section{Radial Problem}

\subsection{Trapping}

In [26, Section 4], we use a Regge-Wheeler change of variables $r \rightarrow x$, under which and after an appropriate rescaling the radial operator becomes (using the notation of (1.12))

$$
\begin{gathered}
P_{x}(h)=h^{2} D_{x}^{2}+V(x, \tilde{\omega}, \tilde{\nu}, \tilde{\lambda}, \tilde{\mu}, \tilde{k} ; h), \\
V(x ; h)=(\tilde{\lambda}+i h \tilde{\mu}) \Delta_{r}-(1+\alpha)^{2}\left(\left(r^{2}+a^{2}\right)(\tilde{\omega}+i h \tilde{\nu})-a \tilde{k}\right)^{2}
\end{gathered}
$$

(note the difference in notation with [26, Section 7]). Let $V(x ; h)=V_{0}(x)+$ $h V_{1}(x)+h^{2} V_{2}(x)$, where

$$
V_{0}(x)=\tilde{\lambda} \Delta_{r}-(1+\alpha)^{2}\left(\left(r^{2}+a^{2}\right) \tilde{\omega}-a \tilde{k}\right)^{2}
$$

is the semiclassical principal part of $V(x)$; note that $V_{0}$ is real-valued and for $1 \leq \tilde{\omega} \leq 2$ and $a$ small enough, $V_{0}( \pm \infty)<0$. Now, [26, Proposition 7.4] establishes an arbitrarily large strip free of radial poles in the nontrapping cases; therefore, the only radial poles in the region (1.13) appear in case (3) of [26, Proposition 7.3]. Using the proof of the latter proposition, we may assume that:

- $\left|\tilde{\lambda}-\tilde{\lambda}_{0}(\tilde{\omega}, \tilde{k})\right|<\varepsilon_{r}$, where $\tilde{\lambda}_{0}^{-1}$ is the value of the function

$$
F_{V}(r ; \tilde{\omega}, \tilde{k})=\frac{\Delta_{r}}{(1+\alpha)^{2}\left(\left(r^{2}+a^{2}\right) \tilde{\omega}-a \tilde{k}\right)^{2}}
$$

at its only maximum point. Under the assumptions (1.13), $1 / C \leq \tilde{\lambda}_{0} \leq C$ for some constant $C$;

- $V_{0}$, as a function of $x$, has unique global maximum $x_{0},\left|V_{0}\left(x_{0}\right)\right|<\varepsilon_{r}^{3}$ and $V_{0}^{\prime \prime}(x)<0$ for $\left|x-x_{0}\right| \leq \varepsilon_{r}$;

- $V_{0}(x)<-\varepsilon_{r}^{3}$ for $\left|x-x_{0}\right| \geq \varepsilon_{r}$.

Here $\varepsilon_{r}>0$ is a small constant we will choose later (Fig. 3). We can also compute

$$
\begin{gathered}
\tilde{\lambda}_{0}=\frac{27 M_{0}^{2} \tilde{\omega}^{2}}{1-9 \Lambda M_{0}^{2}} \quad \text { for } a=0 \\
V_{0}^{\prime \prime}\left(x_{0}\right)=-18 M_{0}^{4}\left(1-9 \Lambda M_{0}^{2}\right)^{2} \tilde{\lambda} \quad \text { for } a=0, \tilde{\lambda}=\tilde{\lambda}_{0} .
\end{gathered}
$$

Letting

$$
p_{0}(x, \xi)=\xi^{2}+V_{0}(x)
$$

be the principal symbol of $P_{x}$, we see that $p_{0}$ has a nondegenerate hyperbolic critical point at $\left(x_{0}, 0\right)$ and this is the only critical point in the set $\left\{p_{0} \geq-\varepsilon_{r}^{3}\right\}$. 


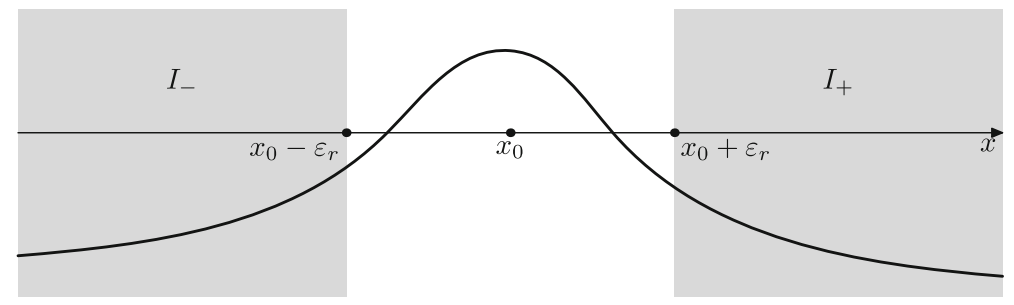

Figure 3. The potential $V_{0}$ and the intervals $I_{ \pm}$

\subsection{WKB Solutions and the Outgoing Condition}

Firstly, we obtain certain approximate solutions to the equation $P_{x} u=0$ in the region $\left|x-x_{0}\right|>\varepsilon_{r}$, where $V_{0}$ is known to be negative. (Compare with [40, Sections 2 and 3].) Define the intervals

$$
I_{+}=\left(x_{0}+\varepsilon_{r},+\infty\right), I_{-}=\left(-\infty, x_{0}-\varepsilon_{r}\right), I_{0}=\left(x_{0}-2 \varepsilon_{r}, x_{0}+2 \varepsilon_{r}\right) .
$$

Let $\psi_{0}(x)$ be a smooth function on $I_{+} \cup I_{-}$solving the eikonal equation

$$
\psi_{0}^{\prime}(x)=\operatorname{sgn}\left(x-x_{0}\right) \sqrt{-V_{0}(x)} .
$$

(We will specify a normalization condition for $\psi_{0}$ later.) Then, we can construct approximate WKB solutions

$$
u_{ \pm}^{+}(x ; h)=e^{i \psi_{0}(x) / h} a_{ \pm}^{+}(x ; h), u_{ \pm}^{-}(x ; h)=e^{-i \psi_{0}(x) / h} a_{ \pm}^{-}(x ; h), x \in I_{ \pm},
$$

such that $P_{x} u_{ \pm}^{\delta}=O\left(h^{\infty}\right)$ in $C^{\infty}\left(I_{ \pm}\right)^{8}$ and $a_{\gamma}^{\delta}$ are smooth classical symbols in $h$, for $\gamma, \delta \in\{+,-\}$. Indeed, if

$$
a_{\gamma}^{\delta}(x ; h) \sim \sum_{j \geq 0} h^{j} a_{\gamma}^{\delta(j)}(x),
$$

then the functions $a_{\gamma}^{\delta(j)}$ have to solve the transport equations

$$
\begin{gathered}
\left(2 \psi_{0}^{\prime}(x) \partial_{x}+\psi_{0}^{\prime \prime}(x) \pm i V_{1}(x)\right) a_{\gamma}^{ \pm(0)}=0 \\
\left(2 \psi_{0}^{\prime}(x) \partial_{x}+\psi_{0}^{\prime \prime}(x) \pm i V_{1}(x)\right) a_{\gamma}^{ \pm(j+1)}= \pm i\left(\partial_{x}^{2}-V_{2}(x)\right) a_{\gamma}^{ \pm(j)}, j \geq 0
\end{gathered}
$$

the latter can be solved inductively in $j$. We will fix the normalization of $a_{\gamma}^{\delta(0)}$ later; right now, we only require that for $x$ in a compact set, $a_{\gamma}^{\delta(0)} \sim 1$ in the sense that $C^{-1} \leq\left|a_{\gamma}^{\delta(0)}\right| \leq C$ for some $h$-independent constant $C$. Put

$$
\Gamma_{\gamma}^{ \pm}=\left\{\left(x, \pm \psi_{0}^{\prime}(x)\right) \mid x \in I_{\gamma}\right\} \subset T^{*} I_{\gamma}, \gamma \in\{+,-\} ;
$$

then, by Proposition 2.1 (with $m=0$ ),

$$
\mathrm{WF}_{h}\left(u_{\gamma}^{\delta}\right) \subset \Gamma_{\gamma}^{\delta}, \gamma, \delta \in\{+,-\}
$$

\footnotetext{
${ }^{8}$ Henceforth we say that $u=O\left(h^{\infty}\right)$ in $C^{\infty}(I)$ for some open set $I$, if for every compact $K \subset I$ and every $N,\|u\|_{C^{N}(K)}=O\left(h^{N}\right)$. In particular, this does not provide any information on the growth of $u$ at the ends of $I$. Similarly, we say that $u$ is polynomially bounded in $C^{\infty}(I)$ if for every $K$ and $N$, there exists $M$ such that $\|u\|_{C^{N}(K)}=O\left(h^{-M}\right)$.
} 
Now, we show that the Cauchy problem for the equation $P_{x} u=0$ is wellposed semiclassically in $I_{ \pm}$. For two smooth functions $v_{1}, v_{2}$ on some interval, define their semiclassical Wronskian by

$$
W\left(v_{1}, v_{2}\right)=v_{1} \cdot h \partial_{x} v_{2}-v_{2} \cdot h \partial_{x} v_{1}
$$

then

$$
h \partial_{x} W\left(v_{1}, v_{2}\right)=v_{2} \cdot P_{x} v_{1}-v_{1} \cdot P_{x} v_{2} .
$$

Also, if $W\left(v_{1}, v_{2}\right) \neq 0$ and $u$ is some smooth function, then

$$
u=\frac{W\left(u, v_{1}\right) v_{2}-W\left(u, v_{2}\right) v_{1}}{W\left(v_{2}, v_{1}\right)} .
$$

We have $W\left(u_{ \pm}^{+}, u_{ \pm}^{-}\right) \sim 1$; therefore, the following fact applies:

Proposition 4.1. Assume that $I \subset \mathbb{R}$ is an interval and $U \subset I$ is a nonempty open set. Let $v_{1}(x ; h), v_{2}(x ; h) \in C^{\infty}(I)$ be two polynomially bounded functions such that $P_{x} v_{j}(x ; h)=O\left(h^{\infty}\right)$ in $C^{\infty}(I)$ and $W\left(v_{1}, v_{2}\right)^{-1}$ is polynomially bounded. (Note that by $(4.7), d_{x} W\left(v_{1}, v_{2}\right)=O\left(h^{\infty}\right)$ ). Let $u(x ; h) \in$ $C^{\infty}(I)$ be polynomially bounded in $C^{\infty}(U)$ and $P_{x} u=O\left(h^{\infty}\right)$ in $C^{\infty}(I)$. Then, $u=c_{1} v_{1}+c_{2} v_{2}+O\left(h^{\infty}\right)$ in $C^{\infty}(I)$, where the constants $c_{1}, c_{2}$ are polynomially bounded. Moreover, $c_{j}=W\left(u, v_{3-j}\right) / W\left(v_{j}, v_{3-j}\right)+O\left(h^{\infty}\right)$.

Proof. Let $W_{j}=W\left(u, v_{j}\right)$. Combining (4.7) and (4.8), we get $\left|d_{x} W_{j}\right|=$ $O\left(h^{\infty}\right)\left(\left|W_{1}\right|+\left|W_{2}\right|\right)$. Also, $W_{j}$ are polynomially bounded on $U$. By Gronwall's inequality, we see that $W_{j}$ are polynomially bounded on $I$ and constant modulo $O\left(h^{\infty}\right)$; it remains to use $(4.8)$.

Now, recall [26, Section 4] that for $X_{0}$ large enough, we have $V(x)=$ $V_{ \pm}\left(e^{\mp A_{ \pm} x}\right)$ for $\pm x>X_{0}$, where $A_{ \pm}>0$ are some constants and $V_{ \pm}(w)$ are holomorphic functions in the disks $\left\{|w|<e^{-A_{ \pm} X_{0}}\right\}$, and $V_{ \pm}(0)=-\omega_{ \pm}^{2}$, where

$$
\omega_{ \pm}=(1+\alpha)\left(\left(r_{ \pm}^{2}+a^{2}\right)(\tilde{\omega}+i h \tilde{\nu})-a \tilde{k}\right) .
$$

For $a$ and $h$ small enough, we have Re $\omega_{ \pm}>0$. In [26, Section 4], we constructed exact solutions $u_{ \pm}(x)$ to the equation $P_{x} u_{ \pm}=0$ such that

$$
u_{ \pm}(x)=e^{ \pm i \omega_{ \pm} x / h} v_{ \pm}\left(e^{\mp A_{ \pm} x}\right) \text { for } \pm x>X_{0},
$$

with $v_{ \pm}(w)$ holomorphic in the disks $\left\{|w|<e^{-A_{ \pm} X_{0}}\right\}$ and $v_{ \pm}(0)=1$. Note that we can use a different normalization condition than [26, Proposition 4.2], as $\operatorname{Im} \omega_{ \pm}=O(h)$ under the assumptions (1.13).

Proposition 4.2. For a certain normalization of the functions $\psi_{0}$ and $a_{ \pm}^{+(0)}$,

$$
u_{ \pm}(x)=u_{ \pm}^{+}(x)+O\left(h^{\infty}\right) \quad \text { in } C^{\infty}\left(I_{ \pm}\right) .
$$

In particular, by (4.6)

$$
\mathrm{WF}_{h}\left(\left.u_{ \pm}\right|_{I_{ \pm}}\right) \subset \Gamma_{ \pm}^{+} .
$$


Proof. We will consider the case of $u_{+}$. By Proposition 4.1, it is enough to show (4.10) for $\pm x>X_{0}$, where $X_{0}$ is large, but fixed. We choose $X_{0}$ large enough so that $\operatorname{Re} V_{ \pm}(w)<0$ for $|w| \leq e^{-A_{ \pm} X_{0}}$. Then, there exists a function $\psi(x)$ such that

$$
\begin{gathered}
\left(\partial_{x} \psi(x)\right)^{2}+V(x)=0, \quad x>X_{0} ; \\
\psi(x)=\omega_{+} x+\tilde{\psi}\left(e^{-A_{+} x}\right),
\end{gathered}
$$

with $\tilde{\psi}$ holomorphic in $\left\{|w|<e^{-A_{+} X_{0}}\right\}$. We can fix $\psi$ by requiring that $\tilde{\psi}(0)=$ 0. Take

$$
u_{+}(x)=e^{i \psi(x) / h} a\left(e^{-A_{+} x} ; h\right) ;
$$

then $P_{x} u_{+}=0$ if and only if

$$
\left(\left[h A_{+} w D_{w}+A_{+} w \tilde{\psi}^{\prime}(w)-\omega_{+}\right]^{2}+V\right) a=0 .
$$

This can be rewritten as

$$
-A_{+}\left(w \tilde{\psi}^{\prime}(w)\right)^{\prime} a+\left(2 \omega_{+}+i h A_{+}-2 A_{+} w \tilde{\psi}^{\prime}(w)\right) \partial_{w} a+i h A_{+} w \partial_{w}^{2} a=0 .
$$

We will solve this equation by a power series in $w$ and estimate the terms of this series uniformly in $h$. Let us write

$$
\tilde{\psi}^{\prime}(w ; h)=\sum_{l \geq 0} \psi_{l}(h) w^{l}, \quad a(w ; h)=\sum_{j \geq 0} a_{j}(h) w^{j}
$$

and solve for $a_{j}$ with the initial condition $a_{0}=1$, obtaining

$$
a_{j+1}(h)=\frac{A_{+}}{(j+1)\left(2 \omega_{+}+i h A_{+}(j+1)\right)} \sum_{0 \leq l \leq j} \psi_{l}(h)(1+2 j-l) a_{j-l}(h) .
$$

We claim that for some $R$, all $j$, and small $h,\left|a_{j}(h)\right| \leq R^{j}$. Indeed, we have $\left|2 \omega_{+}+i h A_{+}(j+1)\right| \geq \varepsilon>0$; combining this with an estimate on $\psi_{l}$, we get

$$
\left|a_{j+1}\right| \leq \frac{C}{j+1} \sum_{0 \leq l \leq j} S^{l}(1+2 j-l)\left|a_{j-l}\right| \leq 2 C \sum_{0 \leq l \leq j} S^{l}\left|a_{j-l}\right|
$$

for some constants $C$ and $S$. We can then conclude by induction if $R \geq 2 C+S$. In a similar way, we can estimate the derivatives of $a_{j}$ in $h$; therefore, $a(w ; h)$ is a classical symbol for $|w|<R^{-1}$.

Now, we take $X_{0}$ large enough so that $e^{-A_{+} X_{0}}<R^{-1}$ and restrict ourselves to real $x>X_{0}$. We can normalize $\psi_{0}$ so that $\psi(x)=\psi_{0}(x)+h \psi_{1}(x ; h)$ for some classical symbol $\psi_{1}$; then

$$
u_{+}(x)=e^{i \psi_{0}(x) / h}\left[e^{i \psi_{1}(x ; h)} a\left(e^{-A_{+} x} ; h\right)\right] .
$$

The expression in square brackets is a classical symbol; therefore, this expression solves the transport Eq. (4.4); it is then equal to a constant times $a_{+}^{+}$, modulo $O\left(h^{\infty}\right)$ errors. 

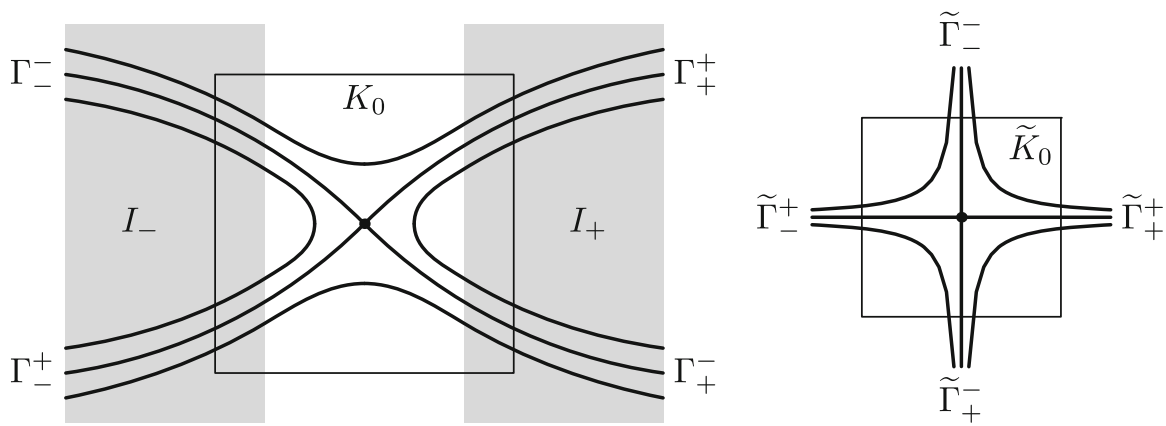

FiguRE 4 . Level lines of $p_{0}$ before and after conjugation by $\Phi$

\subsection{Transmission Through the Barrier}

First of all, we establish a microlocal normal form for $P_{x}$ near the potential maximum. Let $\varepsilon_{0}>0$ be small; define

$$
K_{0}=\left\{\left|x-x_{0}\right| \leq \varepsilon_{0},|\xi| \leq \varepsilon_{0}\right\} \subset T^{*} \mathbb{R} .
$$

We pick $\varepsilon_{r}$ small enough, depending on $\varepsilon_{0}$, such that $\varepsilon_{r}<\varepsilon_{0} / 2$ and

$$
\left\{p_{0}=0\right\} \subset K_{0} \cup \bigcup_{\gamma, \delta} \Gamma_{\gamma}^{\delta},
$$

with $\Gamma_{\gamma}^{\delta}$ defined in (4.5). (Recall from Sect. 4.1 that $\varepsilon_{r}$ controls how close we are to the trapping region). We also assume that $\varepsilon_{0}$ is small enough so that $\left(x_{0}, 0\right)$ is the only critical point of $p_{0}$ in $K_{0}$.

Proposition 4.3. For $\varepsilon_{0}$ small enough and $\varepsilon_{r}$ small enough depending on $\varepsilon_{0}$, there exists a symplectomorphism $\Phi$ (Fig. 4) from a neighborhood of $K_{0}$ onto a neighborhood of the origin in $T^{*} \mathbb{R}$ and operators $B_{1}, B_{2}$ quantizing $\Phi$ near $K_{0} \times \Phi\left(K_{0}\right)$ in the sense of Proposition 2.5, such that $P_{x}$ is intertwined via $\left(B_{1}, B_{2}\right)$ with the operator $S Q(\beta)$ microlocally near $K_{0} \times \Phi\left(K_{0}\right)$, with $S \in \Psi_{\mathrm{cl}}^{0}$ elliptic in the class $\Psi^{0}(\mathbb{R})$,

$$
Q(\beta)=h x D_{x}-\beta,
$$

and $\beta=\beta(\tilde{\omega}, \tilde{\nu}, \tilde{\lambda}, \tilde{\mu}, \tilde{k} ; h)$ a classical symbol. Moreover, the principal part $\beta_{0}$ of $\beta$ is real-valued, independent of $\tilde{\nu}, \tilde{\mu}$, and vanishes if and only $\tilde{\lambda}=\tilde{\lambda}_{0}(\tilde{\omega}, \tilde{k})$. Also,

$$
\begin{gathered}
\beta_{0}=-\frac{V_{0}\left(x_{0}\right)}{\sqrt{-2 V_{0}^{\prime \prime}\left(x_{0}\right)}}+O\left(V_{0}\left(x_{0}\right)^{2}\right), \\
\beta_{0}=\frac{27 M_{0}^{2} \tilde{\omega}^{2}-\tilde{\lambda}\left(1-9 \Lambda M_{0}^{2}\right)}{2 \sqrt{\tilde{\lambda}}\left(1-9 \Lambda M_{0}^{2}\right)}+O\left(V_{0}\left(x_{0}\right)^{2}\right) \quad \text { for } a=0 .
\end{gathered}
$$


Finally, $\Phi\left(K_{0}\right) \subset \widetilde{K}_{0}=\left\{|x| \leq \tilde{\varepsilon}_{0},|\xi| \leq \tilde{\varepsilon}_{0}\right\}$ for some $\tilde{\varepsilon}_{0}$ depending on $\varepsilon_{0}$, and, with $I_{0}$ defined in (4.2),

$$
\begin{gathered}
\Phi\left(\Gamma_{ \pm}^{+}\right) \subset \widetilde{\Gamma}_{ \pm}^{+}=\left\{ \pm x>0, \xi=\beta_{0} / x,|\xi| \leq \tilde{\varepsilon}_{0} / 2\right\}, \\
\Phi\left(\Gamma_{ \pm}^{-}\right) \subset \widetilde{\Gamma}_{ \pm}^{-}=\left\{\mp \xi>0, x=\beta_{0} / \xi,|x| \leq \tilde{\varepsilon}_{0} / 2\right\} ; \\
\left\{p_{0}=0\right\} \cap\left\{x \in I_{0}\right\} \subset \Phi^{-1}\left(\widetilde{K}_{0}\right) \subset K_{0} .
\end{gathered}
$$

Proof. First of all, we use [16, Theorem 12] to construct $\Phi, B_{1}, B_{2}$ conjugating $P_{x}$ microlocally near the critical point $\left(x_{0}, 0\right)$ to an operator of the form $f\left[h x D_{x}\right]$, for some symbol $f(s ; h)$, where the latter employs the formal functional calculus of Sect. 2.2. The techniques in the proof are similar to those of Sect. 3.3 of the present paper, with appropriate replacements for Propositions 2.10 and 3.7; therefore, the proof goes through for complex valued symbols with real principal part.

Let $f_{0}$ be the principal part of $f$; then, $p_{0} \circ \Phi^{-1}=f_{0}(x \xi)$. Note that $\Phi\left(x_{0}, 0\right)=(0,0)$. The level set $\left\{p_{0}=V_{0}\left(x_{0}\right)\right\}$ at the trapped energy contains in particular the outgoing trajectory $\left\{x>x_{0}, \xi=\sqrt{V_{0}\left(x_{0}\right)-V_{0}(x)}\right\}$; we can choose $\Phi$ mapping this trajectory into $\{x>0, \xi=0\}$. Since the latter is also outgoing for the Hamiltonian flow of $x \xi$, we have $\partial_{s} f_{0}(0)>0$; it follows that $\partial_{s} f_{0}(s)>0$ for all $s$ (if $\partial_{s} f_{0}$ vanishes, then $p_{0}$ has a critical point other than $\left.\left(x_{0}, 0\right)\right)$. The function $f(s ; h)$ not uniquely defined; however, its Taylor decomposition at $s=0, h=0$ is and we can compute in particular

$$
f_{0}(s)=V_{0}\left(x_{0}\right)+s \sqrt{-2 V_{0}^{\prime \prime}\left(x_{0}\right)}+O\left(s^{2}\right)
$$

Therefore, for $\varepsilon_{r}$ small enough, we can solve the equation $f(s ; h)=0$ for $s$; let $\beta$ be the solution. We now write $f(s ; h)=f_{1}(s ; h)(s-\beta)$ for some nonvanishing $f_{1}$ and get $f\left[h x D_{x}\right]=S Q(\beta)$ microlocally in $\Phi\left(K_{0}\right)$, with $S=f_{1}\left[h x D_{x}\right]$ in $\Phi\left(K_{0}\right)$ and extended to be globally elliptic outside of this set. The Eq. (4.12) follows from (4.17), while (4.13) follows from (4.12) and (4.1).

Finally, $p_{0}=0$ on each $\Gamma_{\gamma}^{\delta}$ and thus $x \xi=\beta$ on $\Phi\left(\Gamma_{\gamma}^{\delta}\right)$. By analyzing the properties of $\Phi$ near $\left(x_{0}, 0\right)$, we can deduce which part of the sets $\{x \xi=\beta\}$ each $\Gamma_{\gamma}^{\delta}$ maps into; (4.14)-(4.16) follow for $\varepsilon_{r}$ small enough.

We now describe the radial quantization condition and provide a nonrigorous explanation for it. Recall from [26, Section 4] that $(\omega, \lambda, k)$ is a pole of $R_{r}$ if and only if the functions $u_{ \pm}$, studied in the previous subsection, are multiples of each other. Assume that this is true and $u=u_{+} \sim u_{-}$. However, by (4.11) the function $B_{1} u$ is microlocalized on the union of $\widetilde{\Gamma}_{ \pm}^{+}$, but away from $\widetilde{\Gamma}_{ \pm}^{-}$; it also solves $Q(\beta) B_{1} u=0$ microlocally. By propagation of singularities, this can happen only if the characteristic set of $Q(\beta)$ is $\{x \xi=0\}$, and in this case, $B_{1} u$ is smooth near $x=0$. Then, $B_{1} u$ must be given by $x^{i \beta / h}$, with $\beta \in-i h \mathbb{N}$ and $\mathbb{N}$ denoting the set of nonnegative integers. Therefore, we define the radial quantization symbol $\mathcal{F}^{r}(m, \tilde{\omega}, \tilde{\nu}, \tilde{k} ; h)$ as the solution $\tilde{\lambda}+i h \tilde{\mu}$ to the equation

$$
\beta(\tilde{\omega}, \tilde{\nu}, \tilde{\lambda}, \tilde{\mu}, \tilde{k} ; h)=-i h m, \quad m \in \mathbb{Z}, \quad 0 \leq m \leq C_{m} .
$$


The expansions for $\mathcal{F}^{r}$ near $a=0$ described in Proposition 1.5 follow from (4.13) and (B.12).

We now prove the rest of Proposition 1.5. We start with quantifying the statement that in order for the equation $Q(\beta) u=0$ to have a nontrivial solution smooth near $x=0$, the quantization condition must be satisfied:

Proposition 4.4. Assume that $\beta \in \mathbb{C}$ satisfies

$$
|\beta| \leq C_{\beta}, \quad|\operatorname{Im} \beta| \leq C_{\beta} h, \quad \min _{m \in \mathbb{N}}|\beta+i h m| \geq C_{\beta}^{-1} h,
$$

for some constant $C_{\beta}$. Let $U \subset \mathbb{R}$ be a bounded open interval, $I \subset U$ be a compact interval centered at zero, and $X \in \Psi_{\mathrm{cl}}^{\mathrm{comp}}(\mathbb{R})$. Then, there exist constants $C$ and $N$ such that for each $u \in L^{2}(\mathbb{R})$,

$$
\|X u\|_{L^{2}(I)} \leq C h^{-N}\|Q(\beta) X u\|_{L^{2}(U)}+O\left(h^{\infty}\right)\|u\|_{L^{2}(\mathbb{R})} .
$$

Proof. First, assume that $\operatorname{Im} \beta \geq h$. Let $I^{\prime}$ be an interval compactly contained in $I$ and centered at zero. We will use the fact that every $C^{\infty}\left(I^{\prime}\right)$ seminorm of $X u$ is bounded by $C h^{-N}\|X u\|_{L^{2}(I)}+O\left(h^{\infty}\right)\|u\|_{L^{2}(\mathbb{R})}$ for some constants $C$ and $N$, depending on the seminorm chosen. (Henceforth $C$ and $N$ will be constants whose actual values may depend on the context). Since $X u \in C^{\infty}$, we can write

$$
h D_{x}\left(x^{-i \beta / h} X u\right)=x^{-1-i \beta / h} Q(\beta) X u .
$$

However, $\operatorname{Re}(-i \beta / h) \geq 1$; therefore, $x^{-i \beta / h} X u$ vanishes at $x=0$ and we can integrate to get

$$
\left\|x^{-i \beta / h} X u\right\|_{L^{\infty}(I)} \leq C\|Q(\beta) X u\|_{L^{2}(I)} .
$$

On the other hand,

$$
\|X u\|_{L^{\infty}\left(I^{\prime}\right)} \leq C h^{-N_{0}}\|X u\|_{L^{2}(I)}+O\left(h^{\infty}\right)\|u\|_{L^{2}(\mathbb{R})} .
$$

for some constants $C$ and $N_{0}$. Now, take a large constant $\varkappa$; using (4.21) in $\left\{|x|<h^{\varkappa}\right\}$ and (4.20) elsewhere, we get

$\|X u\|_{L^{2}(I)} \leq C h^{\varkappa / 2-N_{0}}\|X u\|_{L^{2}(I)}+h^{-\varkappa C_{\beta}}\|Q(\beta) X u\|_{L^{2}(I)}+O\left(h^{\infty}\right)\|u\|_{L^{2}(\mathbb{R})} ;$

taking $\varkappa$ large enough, we get (4.19).

For the general case, we choose an integer $M$ large enough so that $\operatorname{Im}(\beta+$ $i h M) \geq h$. Let $v$ be $M$ th Taylor polynomial of $X u$ at zero. Since

$$
\begin{aligned}
\| Q & (\beta+i h M) D_{x}^{M} X u \|_{L^{2}(I)} \\
= & \left\|D_{x}^{M} Q(\beta) X u\right\|_{L^{2}(I)} \\
\leq & C h^{-N}\|Q(\beta) X u\|_{L^{2}(U)} \\
& +O\left(h^{\infty}\right)\|u\|_{L^{2}(\mathbb{R})},
\end{aligned}
$$

we apply the current proposition for the case $\operatorname{Im} \beta \geq h$ considered above to get

$\|X u-v\|_{L^{2}(I)} \leq C\left\|D_{x}^{M} X u\right\|_{L^{2}(I)} \leq C h^{-N}\|Q(\beta) X u\|_{L^{2}(U)}+O\left(h^{\infty}\right)\|u\|_{L^{2}(\mathbb{R})} ;$ 
therefore, $\|Q(\beta) v\|_{L^{2}(I)}$ is bounded by the same expression. However, one can verify directly that if $\beta$ is $C_{\beta}^{-1} h$ away from $-i h \mathbb{N}$, then

$$
\|v\|_{L^{2}(I)} \leq C h^{-N}\|Q(\beta) v\|_{L^{2}(I)}
$$

this completes the proof.

Now, we show that each radial pole lies within $o(h)$ of a pseudopole:

Proposition 4.5. Assume that $\beta(h)$ satisfies (4.18). Then, for $h$ small enough, $(\omega, \lambda, k)$ is not a radial pole, and for each compact interval $I \subset \mathbb{R}$, there exist constants $C$ and $N$ such that

$$
\left\|1_{I} R_{r}(\omega, \lambda, k) 1_{I}\right\|_{L^{2} \rightarrow L^{2}} \leq C h^{-N} .
$$

Proof. Let $u \in H_{\text {loc }}^{2}(\mathbb{R})$ be an outgoing solution to the equation $P_{x} u=f$, with $f \in L^{2}$ supported in a fixed compact subset inside the open interval $I$. Then

$$
u(x)=c_{ \pm} u_{ \pm}(x), \quad \pm x \gg 0,
$$

for some constants $c_{ \pm}$. Clearly, $\left|c_{ \pm}\right| \leq C\|u\|_{L^{2}(I)}$. Using the method of proof of Proposition 4.1, we get

$$
\left\|u-c_{ \pm} u_{ \pm}\right\|_{L^{2}\left(I \cap I_{ \pm}\right)} \leq C h^{-1}\|f\|_{L^{2}} .
$$

Next, let $\chi \in C_{0}^{\infty}\left(I_{0}\right)$ be equal to 1 near the complement of $I_{+} \cup I_{-}$and $B_{1}$ be the operator introduced in Proposition 4.3; consider the compactly microlocalized operator

$$
T=S Q(\beta) B_{1}-B_{1} P_{x} .
$$

Then, by Proposition 2.5, we can write $T=T X+O\left(h^{\infty}\right)$, where $X \in \Psi_{\mathrm{cl}}^{\text {comp }}$ is a certain operator vanishing microlocally on $K_{0}$. By (4.16), we can further write $X=X_{1}+X_{2}$, where $X_{j} \in \Psi_{\mathrm{cl}}^{\mathrm{comp}}, \mathrm{WF}_{h}\left(X_{j}\right) \cap K_{0}=\emptyset, \mathrm{WF}_{h}\left(X_{1}\right) \cap\left\{p_{0}=0\right\}=\emptyset$ and $\mathrm{WF}_{h}\left(X_{2}\right) \subset\left\{x \in I_{+} \cup I_{-}\right\}$. By ellipticity,

$$
\left\|X_{1} u\right\|_{L^{2}} \leq C\|f\|_{L^{2}}+O\left(h^{\infty}\right)\|u\|_{L^{2}(I)} .
$$

Take $\chi_{ \pm} \in C_{0}^{\infty}\left(I_{ \pm}\right)$such that $\chi_{ \pm}=1$ near $I_{ \pm} \cap \pi\left(\mathrm{WF}_{h}\left(X_{2}\right)\right)$ (here $\pi: \mathbb{T}^{*} \mathbb{R} \rightarrow$ $\mathbb{R}$ is the projection map onto the base variable and $\pi\left(\mathrm{WF}_{h}\left(X_{2}\right)\right)$ is a compact subset of $\left.I_{+} \cup I_{-}\right)$; then, by (4.22),

$$
\begin{gathered}
\left\|X_{2}\left(u-u_{1}\right)\right\|_{L^{2}} \leq C h^{-1}\|f\|_{L^{2}}+O\left(h^{\infty}\right)\|u\|_{L^{2}(I)}, \\
u_{1}=c_{+} \chi_{+}(x) u_{+}+c_{-} \chi_{-}(x) u_{-} .
\end{gathered}
$$

It follows that

$$
\left\|S Q(\beta) B_{1} u-T X_{2} u_{1}\right\|_{L^{2}} \leq C h^{-1}\|f\|_{L^{2}}+O\left(h^{\infty}\right)\|u\|_{L^{2}(I)} .
$$

Combining (4.11) with (4.14) and the fact that $\mathrm{WF}_{h}\left(X_{2}\right) \cap K_{0}=\emptyset$, we get

$$
\mathrm{WF}_{h}\left(T X_{2} \chi_{ \pm}(x) u_{ \pm}\right) \subset \widetilde{\Gamma}_{ \pm}^{+} \backslash \widetilde{K}_{0} .
$$

The projections of the latter sets onto the $x$ variable do not intersect $\tilde{I}_{0}=$ $\left\{|x| \leq \tilde{\varepsilon}_{0}\right\}$; therefore, for some open $\widetilde{U}_{0}$ containing $\tilde{I}_{0}$,

$$
\left\|T X_{2} u_{1}\right\|_{L^{2}\left(\widetilde{U}_{0}\right)}=O\left(h^{\infty}\right)\|u\|_{L^{2}(I)} .
$$


Using ellipticity of $S$, we then get

$$
\left\|Q(\beta) B_{1} u\right\|_{L^{2}\left(\widetilde{U}_{0}\right)} \leq C h^{-1}\|f\|_{L^{2}}+O\left(h^{\infty}\right)\|u\|_{L^{2}(I)} .
$$

Applying Proposition 4.4 to $B_{1} u$ on $I_{0}$ and using that $B_{1}$ is compactly microlocalized, we get

$$
\left\|\widetilde{X} B_{1} u\right\|_{L^{2}} \leq C h^{-N}\|f\|_{L^{2}}+O\left(h^{\infty}\right)\|u\|_{L^{2}(I)},
$$

for any $\tilde{X} \in \Psi_{\mathrm{cl}}^{\text {comp }}$ microlocalized in $\widetilde{K}_{0}$. Using the elliptic estimate and (4.16), we get

$$
\|u\|_{L^{2}\left(I_{0}\right)} \leq C h^{-N}\|f\|_{L^{2}}+O\left(h^{\infty}\right)\|u\|_{L^{2}(I)} .
$$

From here by (4.22),

$$
\left|c_{ \pm}\right| \leq C\left\|c_{ \pm} u_{ \pm}\right\|_{L^{2}\left(I_{ \pm} \cap I_{0}\right)} \leq C h^{-N}\|f\|_{L^{2}}+O\left(h^{\infty}\right)\|u\|_{L^{2}(I)} ;
$$

combining the last two estimates with (4.22), we get the required estimate:

$$
\|u\|_{L^{2}(I)} \leq C h^{-N}\|f\|_{L^{2}}+O\left(h^{\infty}\right)\|u\|_{L^{2}(I)} .
$$

To finish the proof of Proposition 1.5, it remains to show

Proposition 4.6. Fix $\tilde{\omega}, \tilde{\nu}, \tilde{k}$ satisfying (1.13), $m \in \mathbb{N}$ bounded by a large constant $C_{m}$, and let $V$ be the set of all $\lambda$ such that

$$
|\beta(\tilde{\omega}, \tilde{\nu}, \tilde{k}, \tilde{\lambda}, \tilde{\mu} ; h)+i h m|<h / 3 .
$$

Then, for $h$ small enough, $R_{r}(\omega, \lambda, k)$ has a unique pole $\lambda_{0}$ in $V$, and $\lambda_{0}$ is within $O\left(h^{\infty}\right)$ of $\mathcal{F}^{r}(m, \tilde{\omega}, \tilde{\nu}, \tilde{k} ; h)$. Moreover, we can write

$$
R_{r}(\omega, \lambda, k)=\frac{S(\lambda)}{\lambda-\lambda_{0}}, \quad \lambda \in V
$$

where the family of operators $S(\lambda): L_{\text {comp }}^{2}(\mathbb{R}) \rightarrow L_{\text {loc }}^{2}(\mathbb{R})$ is bounded polynomially in $h$ and $S\left(\lambda_{0}\right)$ is a rank one operator.

Proof. We will use Proposition 4.3 and the fact that $\beta=O(h)$ to extend the WKB solutions $u_{ \pm}^{+}$from $I_{ \pm}$to the whole $\mathbb{R}$. Consider the locally integrable functions

$$
\tilde{u}_{ \pm}^{+}=(x \pm i 0)^{i \beta / h} .
$$

solving the equation $Q(\beta) \tilde{u}_{ \pm}^{+}=0$. (See for example [34, Section 3.2] for the definition and basic properties of $(x \pm i 0)^{b}$.) We have

$$
\mathrm{WF}_{h}\left(\tilde{u}_{ \pm}^{+}\right) \subset\{\xi=0\} \cup\{x=0, \pm \xi>0\} ;
$$

$\tilde{u}_{ \pm}^{+}(x)=x^{i \beta / h}$ microlocally near $\{x>0, \xi=0\}, \tilde{u}_{ \pm}^{+}(x)=e^{\mp \pi \beta / h}(-x)^{i \beta / h}$ microlocally near $\{x<0, \xi=0\}$. Using the formulas for the Fourier transform of $\tilde{u}_{ \pm}^{+}[34$, Example 7.1.17], we get

$$
\tilde{u}_{ \pm}^{+}(x)=\frac{h^{i \beta / h} e^{\mp \beta \pi /(2 h)}}{\Gamma(-i \beta / h)} \int_{0}^{\infty} \chi(\xi) \xi^{-1-i \beta / h} e^{ \pm i x \xi / h} \mathrm{~d} \xi
$$


microlocally near $\left\{x=0, \pm \xi \in K_{\xi}\right\}$, for every $\chi \in C_{0}^{\infty}(0, \infty)$ such that $\chi=1$ near $K_{\xi} \subset(0, \infty)$. Let $B_{2}$ be the operator constructed in Proposition 4.3. By (4.16) $P_{x} B_{2} \tilde{u}_{ \pm}^{+}=O\left(h^{\infty}\right)$ in $C^{\infty}\left(I_{0}\right)$, and $B_{2} \tilde{u}_{ \pm}^{+}=\tilde{c}_{ \pm} u_{ \pm}^{+}+O\left(h^{\infty}\right)$ in $C^{\infty}\left(I_{ \pm} \cap I_{0}\right)$ for some constants $\tilde{c}_{ \pm} \sim 1$. To prove the latter, we can use the theory of Fourier integral operators and Lagrangian distributions to represent $B_{2} \tilde{u}_{ \pm}^{+}$in the form (4.3) microlocally near $\Gamma_{ \pm}^{+}$; the symbols in these WKB expressions will have to solve the transport equations. Then, we can use $B_{2} \tilde{u}_{ \pm}^{+}$ to extend $u_{ \pm}^{+}$to $I_{ \pm} \cup I_{0}$ so that $P_{x} u_{ \pm}^{+}=O\left(h^{\infty}\right)$ there. We claim that

$$
u_{ \pm}^{+}=c_{ \pm 1} u_{\mp}^{+}+c_{ \pm 2} \Gamma(-i \beta / h)^{-1} u_{\mp}^{-}+O\left(h^{\infty}\right)
$$

in $C^{\infty}\left(I_{\mp} \cap I_{0}\right)$, with $c_{ \pm j}$ constants such that $c_{ \pm j}$ and $c_{ \pm j}^{-1}$ are polynomially bounded in $h$. To show (4.23), we can apply the theory of Lagrangian distributions to $B_{2} \tilde{u}_{ \pm}^{+}$one more time; alternatively, we know that this function is an $O\left(h^{\infty}\right)$ approximate solution to the equation $P_{x} u=0$ on $I_{0} \cap I_{\mp}$, and we have control on its $L^{2}$ norm when microlocalized to $\Gamma_{\mp}^{+}$and $\Gamma_{\mp}^{-}$. Thus, we can extend $u_{ \pm}^{+}$to the whole $\mathbb{R}$ as a polynomially bounded family with $P_{x} u_{ \pm}^{+}=O\left(h^{\infty}\right)$ in $C^{\infty}(\mathbb{R})$ and (4.23) holding on $I_{\mp}$. Similarly we can extend $u_{ \pm}^{-}$; using either of the families $\left(u_{ \pm}^{+}, u_{ \pm}^{-}\right)$in Proposition 4.1 together with Proposition 4.2, we get $u_{ \pm}=u_{ \pm}^{+}+O\left(h^{\infty}\right)$ in $C^{\infty}(\mathbb{R})$. It now follows from (4.23) that

$$
W\left(u_{+}, u_{-}\right)=c\left(\Gamma(-i \beta / h)^{-1}+O\left(h^{\infty}\right)\right),
$$

with $c$ and $c^{-1}$ bounded polynomially in $h$. By [26, (4.8)], we get

$$
R_{r}(\omega, \lambda, k)=\frac{\widetilde{S}(\omega, \lambda, k)}{W\left(u_{+}, u_{-}\right)}
$$

with the family $\widetilde{S}$ holomorphic and bounded polynomially in $h$. Moreover, for $W\left(u_{+}, u_{-}\right)=0, \widetilde{S}$ is proportional to $u_{+} \otimes u_{+}$and thus has rank one. We are now done if we let $\lambda_{0}$ be the unique solution to the equation $W\left(u_{+}, u_{-}\right)=0$ in $V$.

\section{Acknowledgements}

I would like to thank Maciej Zworski for suggesting this problem, helpful advice, and constant encouragement. I would also like to thank András Vasy for sharing with me early versions of [50] and his interest in the project, Michael Hitrik for guiding me through the complexities of [31], and Kiril Datchev, Hamid Hezari, and Jakub Kominiarczuk for friendly consultations and references. I am especially grateful to Emanuele Berti and Vitor Cardoso for providing the data on quasi-normal modes for the Kerr metric in the case of scalar perturbations, used in Appendix B.1. Finally, I would like to thank an anonymous referee for carefully reading the manuscript and many suggestions for improving it. 


\section{Appendix A. Grushin Problems for Several Commuting Operators}

\section{A.1. Global Grushin Problem}

Assume that $P_{1}, \ldots, P_{n}$ are pseudodifferential operators on a compact manifold $M$, with $P_{j} \in \Psi^{k_{j}}(M)$ and $k_{j} \geq 0$.

Definition A.1. We say that $\lambda=\left(\lambda_{1}, \ldots, \lambda_{n}\right) \in \mathbb{C}^{n}$ belongs to the joint spectrum $^{9}$ of $P_{1}, \ldots, P_{n}$, if the joint eigenspace

$$
\left\{u \in C^{\infty}(M) \mid P_{j} u=\lambda_{j} u, j=1, \ldots, n\right\}
$$

is nontrivial. (In our situation, one of the operators $P_{j}$ will be elliptic outside of a compact set, so all joint eigenfunctions will be smooth).

The goal of this appendix is to extract information about the joint spectrum of $P_{1}, \ldots, P_{n}$ from certain microlocal information. Essentially, we will construct exact joint eigenfunctions based on approximate eigenfunctions and certain invertibility conditions. The latter will be given in the form of operators $A_{1}, \ldots, A_{n}$, with the following properties:

(G1) Each $A_{j}$ can be represented as $A_{j}^{\prime}+A_{j}^{\prime \prime}$, where $A_{j}^{\prime}$ is compactly microlocalized and has operator norm $O\left(h^{-r}\right) ; A_{j}^{\prime \prime} \in h^{-r} \Psi^{-k_{j}}(M)$. Here $r>0$ is a constant.

(G2) The commutator of any two of the operators $P_{1}, \ldots, P_{n}, A_{1}, \ldots, A_{n}$ lies in $h^{\infty} \Psi^{-\infty}(M)$.

We would like to describe the joint spectrum of $P_{1}, \ldots, P_{n}$ in a ball of radius $o\left(h^{r}\right)$ centered at zero. First, we consider a situation when there is no joint spectrum:

Proposition A.1. Assume that conditions (G1) and (G2) hold and additionally,

$$
\sum_{j=1}^{n} A_{j} P_{j}=I \quad \bmod h^{\infty} \Psi^{-\infty}(M) .
$$

Then, there exists $\delta>0$ such that for $h$ small enough, the ball of radius $\delta h^{r}$ centered at zero contains no joint eigenvalues of $P_{1}, \ldots, P_{n}$.

Proof. Assume that $u \in L^{2}(M)$ and $P_{j} u=\lambda_{j} u$, where $\left|\lambda_{j}\right| \leq \delta h^{r}$. Then

$$
0=\sum_{j=1}^{n} A_{j}\left(P_{j}-\lambda_{j}\right) u=\left(I+h^{\infty} \Psi^{-\infty}\right) u-\sum_{j=1}^{n} \lambda_{j} A_{j} u .
$$

It follows from condition (G1) that $\left\|A_{j}\right\|_{L^{2} \rightarrow L^{2}}=O\left(h^{-r}\right)$; therefore,

$$
\|u\|_{L^{2}}=O\left(\delta+h^{\infty}\right)\|u\|_{L^{2}}
$$

and we must have $u=0$ for $\delta$ and $h$ small enough.

\footnotetext{
${ }^{9}$ Strictly speaking, this is the definition of the joint point spectrum. However, the operators we study in Sect. 3 are joint elliptic near the fiber infinity, as in Proposition A.4, thus all joint spectrum is given by eigenvalues.
} 
Now, we study the case when the joint spectrum is nonempty. Assume that $S_{1}: \mathbb{C} \rightarrow C^{\infty}(M)$ and $S_{2}: \mathcal{D}^{\prime}(M) \rightarrow \mathbb{C}$ are operators with the following properties:

(G3) Each $S_{j}$ is compactly microlocalized with operator norm $O(1)$.

(G4) $S_{2} S_{1}=1+O\left(h^{\infty}\right)$.

(G5) If $Q$ is any of the operators $P_{1}, \ldots, P_{n}, A_{1}, \ldots, A_{n}$, then $Q S_{1} \in h^{\infty} \Psi^{-\infty}$ and $S_{2} Q \in h^{\infty} \Psi^{-\infty}$.

(G6) We have

$$
\sum_{j=1}^{n} A_{j} P_{j}=I-S_{1} S_{2} \bmod h^{\infty} \Psi^{-\infty}(M) .
$$

Note that (G5) implies that the image of $S_{1}$ consists of $O\left(h^{\infty}\right)$-approximate joint eigenfunctions. For $n=1$, one recovers existence of exact eigenfunctions from approximate ones using Grushin problems, based on Schur complement formula; see for example [31, Section 6]. The proposition below constructs an analog of these Grushin problems for the case of several operators. This construction is more involved, since we need to combine the fact that $P_{j}$ commute exactly, needed for the existence of joint spectrum, with microlocal assumptions (G1)-(G6) having $O\left(h^{\infty}\right)$ error. Note also that condition (G2) does not appear in the case $n=1$.

Proposition A.2. Assume that the conditions (G1)-(G6) hold and the operators $P_{1}, \ldots, P_{n}$ commute exactly; that is, $\left[P_{j}, P_{k}\right]=0$ for all $j, k$. Then, there exists $\delta>0$ such that for $h$ small enough, the ball of radius $\delta h^{r}$ contains exactly one joint eigenvalue of $P_{1}, \ldots, P_{n}$. Moreover, this eigenvalue is $O\left(h^{\infty}\right)$ and the corresponding eigenspace is one dimensional.

Proof. We prove the proposition in the case $n=2$ (which is the case we will need in the present paper); the proof in the general case can be found in Appendix A.3.

For $\lambda=\left(\lambda_{1}, \lambda_{2}\right) \in \mathbb{C}^{2}$, consider the operator

$$
\begin{gathered}
T(\lambda)=\left(\begin{array}{cccc}
P_{1}-\lambda_{1} & -A_{2} & S_{1} & 0 \\
P_{2}-\lambda_{2} & A_{1} & 0 & S_{1} \\
S_{2} & 0 & 0 & 0 \\
0 & S_{2} & 0 & 0
\end{array}\right): \mathcal{H}_{1} \rightarrow \mathcal{H}_{2} ; \\
\mathcal{H}_{1}=L^{2}(M) \oplus H_{h}^{-k_{1}-k_{2}}(M) \oplus \mathbb{C}^{2}, \mathcal{H}_{2}=H_{h}^{-k_{1}}(M) \oplus H_{h}^{-k_{2}}(M) \oplus \mathbb{C}^{2} .
\end{gathered}
$$

The conditions (G1)-(G6) imply that for

$$
Q=\left(\begin{array}{cccc}
A_{1} & A_{2} & S_{1} & 0 \\
-P_{2} & P_{1} & 0 & S_{1} \\
S_{2} & 0 & 0 & 0 \\
0 & S_{2} & 0 & 0
\end{array}\right): \mathcal{H}_{2} \rightarrow \mathcal{H}_{1}
$$

we have $T(0) Q=I+O_{\mathcal{H}_{2} \rightarrow \mathcal{H}_{2}}\left(h^{\infty}\right), Q T(0)=I+O_{\mathcal{H}_{1} \rightarrow \mathcal{H}_{1}}\left(h^{\infty}\right)$. By (G1) and (G3), we have $\|Q\|_{\mathcal{H}_{2} \rightarrow \mathcal{H}_{1}}=O\left(h^{-r}\right)$; therefore, if $\delta>0$ and $h$ are small enough and $|\lambda| \leq \delta h^{r}$, then $T(\lambda)$ is invertible and 


$$
\left\|T(\lambda)^{-1}\right\|_{\mathcal{H}_{2} \rightarrow \mathcal{H}_{1}}=O\left(h^{-r}\right) .
$$

Now, let $|\lambda| \leq \delta h^{r}$ and put

$$
\left(u(\lambda), u_{2}(\lambda), f(\lambda)\right)=T(\lambda)^{-1}(0,0,1,0), f(\lambda)=\left(f_{1}(\lambda), f_{2}(\lambda)\right) \in \mathbb{C}^{2} .
$$

This is the only solution to the following system of equations, which we call global Grushin problem:

$$
\begin{aligned}
\left(P_{1}-\lambda_{1}\right) u(\lambda)-A_{2} u_{2}(\lambda)+S_{1} f_{1}(\lambda) & =0, \\
\left(P_{2}-\lambda_{2}\right) u(\lambda)+A_{1} u_{2}(\lambda)+S_{1} f_{2}(\lambda) & =0, \\
S_{2} u(\lambda) & =1, \\
S_{2} u_{2}(\lambda) & =0 .
\end{aligned}
$$

We claim that $\lambda$ is an element of the joint spectrum if and only if $f(\lambda)=0$, and in that case, the joint eigenspace is one dimensional and spanned by $u(\lambda)$. First, assume that $u$ is a joint eigenfunction with the eigenvalue $\lambda$. Then, $T(\lambda)(u, 0,0,0)=(0,0, s, 0)$, where $s$ is some nonzero number; it follows immediately that $f(\lambda)=0$ and $u$ is a multiple of $u(\lambda)$.

Now, assume that $f(\lambda)=0$; we need to prove that $u(\lambda)$ is a joint eigenfunction for the eigenvalue $\lambda$. By (A.1) and (A.2), it suffices to show that $u_{2}(\lambda)=0$. For that, we multiply (A.2) by $P_{1}-\lambda_{1}$ and subtract (A.1) multiplied by $P_{2}-\lambda_{2} ;$ since $f(\lambda)=0$ and $\left[P_{1}, P_{2}\right]=0$, we get

$$
\left(\left(P_{1}-\lambda_{1}\right) A_{1}+\left(P_{2}-\lambda_{2}\right) A_{2}\right) u_{2}(\lambda)=0 .
$$

Recalling (G6), we get

$$
\left(I-S_{1} S_{2}+O_{H_{h}^{-k_{1}-k_{2}} \rightarrow H_{h}^{-k_{1}-k_{2}}}\left(\delta+h^{\infty}\right)\right) u_{2}(\lambda)=0 .
$$

By (A.4), $\left(I+O\left(\delta+h^{\infty}\right)\right) u_{2}(\lambda)=0$ and thus $u_{2}(\lambda)=0$. The claim is proven.

It remains to show that the equation $f(\lambda)=0$ has exactly one root in the disk of radius $\delta h^{r}$ centered at zero, and this root is $O\left(h^{\infty}\right)$. For that, let $Q T(\lambda)=I-R(\lambda)$; we have

$$
\begin{gathered}
R(\lambda)=\left(\begin{array}{cccc}
\lambda_{1} A_{1}+\lambda_{2} A_{2} & 0 & 0 & 0 \\
-\lambda_{1} P_{2}+\lambda_{2} P_{1} & 0 & 0 & 0 \\
\lambda_{1} S_{2} & 0 & 0 & 0 \\
\lambda_{2} S_{2} & 0 & 0 & 0
\end{array}\right)+O_{\mathcal{H}_{1} \rightarrow \mathcal{H}_{1}}\left(h^{\infty}\right) ; \\
T(\lambda)^{-1}=\left(I+R(\lambda)+(I-R(\lambda))^{-1} R(\lambda)^{2}\right) Q .
\end{gathered}
$$

One can verify that $R(\lambda)^{2} Q(0,0,1,0)=O_{\mathcal{H}_{1}}\left(h^{\infty}\right)$ and then

$$
f(\lambda)=\lambda-g(\lambda ; h),
$$

where $g(\lambda ; h)=O\left(h^{\infty}\right)$ uniformly in $\lambda$. It remains to apply the contraction mapping principle.

Finally, we establish a connection between global Grushin problem and meromorphic resolvent expansions, using some more information about our particular application: 
Proposition A.3. Assume that $n=2, P_{1}, P_{2}$ satisfy the properties stated in the beginning of this subsection, $\left[P_{1}, P_{2}\right]=0, k_{1}>0$, and $P_{1}-\lambda$ is elliptic in the class $\Psi^{k_{1}}$ for some $\lambda \in \mathbb{C}$. If $V$ is the kernel of $P_{2}$, then by analytic Fredholm theory (see for example [54, Theorem D.4]), the resolvent

$$
R(\lambda)=\left.\left(P_{1}-\lambda\right)^{-1}\right|_{V}: H_{h}^{-k_{1}}(M) \cap V \rightarrow L^{2}(M) \cap V
$$

is a meromorphic family of operators in $\lambda \in \mathbb{C}$ with poles of finite rank. Then:

1. Assume that the conditions of Proposition A.1 hold and let $\delta>0$ be given by this proposition. Then, for $h$ small enough, $R(\lambda)$ is holomorphic in $\left\{|\lambda|<\delta h^{r}\right\}$ and $\|R(\lambda)\|_{L^{2} \cap V \rightarrow L^{2}}=O\left(h^{-r}\right)$ in this region.

2. Assume that the conditions of Proposition A.2 hold and let $\left(\lambda_{0}, \lambda_{2}^{0}\right)$ be the joint eigenvalue and $\delta>0$ the constant given by this proposition. Suppose that $\lambda_{2}^{0}=0$. Then for $h$ small enough,

$$
R(\lambda)=S(\lambda)+\frac{\Pi}{\lambda-\lambda_{0}},|\lambda|<\delta h^{r},
$$

where $S(\lambda)$ is holomorphic, $\Pi$ is a rank one operator, and the $L^{2} \cap V \rightarrow L^{2}$ norms of $S(\lambda)$ and $\Pi$ are $O\left(h^{-N}\right)$ for some constant $N$.

Proof. 1. We have

$$
A_{1}\left(P_{1}-\lambda\right)=I-\lambda A_{1}+h^{\infty} \Psi^{-\infty}(M) \text { on } V
$$

the right-hand side is invertible for $\delta$ small enough. Therefore, $R$ has norm $O\left(h^{-r}\right)$.

2. We know that $R(\lambda)$ has a pole at $\lambda$ if and only if there exists nonzero $u \in L^{2}(M) \cap V$ such that $\left(P_{1}-\lambda\right) u=0$; that is, a joint eigenfunction of $\left(P_{1}, P_{2}\right)$ with joint eigenvalue $(\lambda, 0)$. Therefore, $\lambda_{0}$ is the only pole of $R(\lambda)$ in $\left\{|\lambda|<\delta h^{r}\right\}$.

Now, take $\lambda \neq \lambda_{0},|\lambda|<\delta h^{r}$, and assume that $v \in H_{h}^{-k_{1}}(M) \cap V$ and $u=R(\lambda) v \in L^{2}(M) \cap V$. Let $T(\lambda)$ be the family of operators introduced in the proof of Proposition A.2, with $\lambda_{1}=\lambda$ and $\lambda_{2}=0$; we know that $T(\lambda)$ is invertible. We represent $T(\lambda)^{-1}$ as a $4 \times 4$ operator-valued matrix; let $T_{i j}^{-1}(\lambda)$ be its entries. We have $T(\lambda)(u, 0,0,0)=(v, 0, c, 0)$ for some number $c$. However, then $(u, 0,0,0)=T(\lambda)^{-1}(v, 0, c, 0)$; taking the third entry of this equality, we get $T_{31}^{-1}(\lambda) v+T_{33}^{-1}(\lambda) c=0$. Now, $T_{33}^{-1}(\lambda)=f_{1}(\lambda)$, with the latter introduced in the proof of Proposition A.2. Therefore, we can compute $c$ in terms of $v$; substituting this into the expression for $u$, we get the following version of the Schur complement formula:

$$
R(\lambda)=\left.\left(T_{11}^{-1}(\lambda)-\frac{T_{13}^{-1}(\lambda) T_{31}^{-1}(\lambda)}{f_{1}(\lambda)}\right)\right|_{V} .
$$

Next, by the proof of Proposition A.2, $f_{1}\left(\lambda_{0}\right)=0$ and $f_{1}(\lambda)=\lambda+O\left(h^{\infty}\right)$. Therefore, we may write $f_{1}(\lambda)=\left(\lambda-\lambda_{0}\right) / g(\lambda)$, with $g$ holomorphic and bounded by $O(1)$. Let $u_{0}$ be the joint eigenfunction of $\left(P_{1}, P_{2}\right)$ with eigenvalue $\left(\lambda_{0}, 0\right)$; then, $\Pi=-g\left(\lambda_{0}\right) T_{13}^{-1}\left(\lambda_{0}\right) T_{31}^{-1}\left(\lambda_{0}\right)$ is a rank one operator, as $T_{13}^{-1}\left(\lambda_{0}\right)$ acts $\mathbb{C} \rightarrow V$ and $\Pi u_{0}=-\left(1+O\left(h^{\infty}\right)\right) u_{0}$. Since the operators $T_{i j}^{-1}$ are polynomially bounded in $h$, we are done. 


\section{A.2. Local Grushin Problem}

In this subsection, we show how to obtain information about the joint spectrum of two operators $P_{1}, P_{2}$ based only on their behavior microlocally near the set where neither of them is elliptic. For that, we use global Grushin problems discussed in the previous subsection. Assume that $P_{1} \in \Psi_{\mathrm{cl}}^{k_{1}}(M), P_{2} \in \Psi_{\mathrm{cl}}^{k_{2}}(M)$ satisfy

(E1) The principal symbol $p_{j 0}$ of $P_{j}$ is real-valued.

(E2) The symbol $p_{10}$ is elliptic in the class $S^{k_{1}}(M)$ outside of some compact set. As a corollary, the set

$$
K=\left\{(x, \xi) \in T^{*} M \mid p_{10}(x, \xi)=p_{20}(x, \xi)=0\right\}
$$

is compact.

Next, assume that $A_{1}, A_{2}$ are compactly microlocalized operators on $M$ such that:

(L1) For each $j$ and every bounded neighborhood $U$ of $K, A_{j}$ can be represented as $A_{j}^{\prime}+A_{j}^{\prime \prime}$, where both $A_{j}^{\prime}$ and $A_{j}^{\prime \prime}$ are compactly microlocalized, $\left\|A_{j}^{\prime}\right\|=O\left(h^{-r}\right), \mathrm{WF}_{h}\left(A_{j}^{\prime}\right) \subset U \times U$, and $A_{j}^{\prime \prime} \in h^{-r} \Psi_{\mathrm{cl}}^{\mathrm{comp}}(M)$. Here $r \geq 0$ is some constant.

(L2) The commutator of any two of the operators $P_{1}, P_{2}, A_{1}, A_{2}$ lies in $h^{\infty} \Psi^{-\infty}(M)$.

Finally, let $S_{1}: \mathbb{C} \rightarrow C^{\infty}(M), S_{2}: \mathcal{D}^{\prime}(M) \rightarrow \mathbb{C}$ be compactly microlocalized operators such that:

(L3) $\left\|S_{j}\right\|=O(1)$ and $\mathrm{WF}_{h}\left(S_{j}\right) \subset K$.

(L4) $S_{2} S_{1}=1+O\left(h^{\infty}\right)$.

(L5) If $Q$ is any of the operators $P_{1}, P_{2}, A_{1}, A_{2}$, then $Q S_{1} \in h^{\infty} \Psi^{-\infty}$ and $S_{2} Q \in h^{\infty} \Psi^{-\infty}$.

Proposition A.4. 1. If the conditions (E1)-(E2) and (L1)-(L2) hold, and

$$
A_{1} P_{1}+A_{2} P_{2}=I
$$

microlocally near $K \times K$, then there exists $\delta>0$ such that for $h$ small enough, there are no joint eigenvalues of $P_{1}, P_{2}$ in the ball of radius $\delta h^{r}$ centered at zero.

2. If the conditions (E1)-(E2) and (L1) $-(\mathrm{L} 5)$ hold, $\left[P_{1}, P_{2}\right]=0$, and

$$
A_{1} P_{1}+A_{2} P_{2}=I-S_{1} S_{2}
$$

microlocally near $K \times K$, then there exists $\delta>0$ such that for $h$ small enough, the ball of radius $\delta h^{r}$ centered at zero contains exactly one joint eigenvalue $\lambda$ of $P_{1}, P_{2}$. Moreover, $\lambda=O\left(h^{\infty}\right)$ and the corresponding joint eigenspace is one dimensional.

Proof. We will prove part 2; part 1 is handled similarly. Take small $\varepsilon>0$ and let $\chi_{\varepsilon} \in C_{0}^{\infty}(\mathbb{R})$ be supported in $(-\varepsilon, \varepsilon)$ and equal to 1 on $[-\varepsilon / 2, \varepsilon / 2]$. Also, let $\psi_{\varepsilon} \in C^{\infty}(\mathbb{R})$ satisfy $t \psi_{\varepsilon}(t)=1-\chi_{\varepsilon}(t)$ for all $t$; then, $\psi_{\varepsilon}(t)=0$ for $|t| \leq \varepsilon / 2$. The function $\psi_{\varepsilon}$ is a symbol of order -1 , as it is equal to $t^{-1}$ for $|t| \geq \varepsilon$. 
By $(\mathrm{E} 1)$, we can define the operators $\chi_{\varepsilon}\left[P_{j}\right], \psi_{\varepsilon}\left[P_{j}\right] \in \Psi_{\mathrm{cl}}^{\mathrm{loc}}\left(T^{*} M\right)$ using the formal functional calculus introduced in Sect. 2.2. By (E2) and Proposition $2.4 \psi_{\varepsilon}\left[P_{1}\right] \in \Psi_{\mathrm{cl}}^{-k_{1}}(M)$, and $\chi_{\varepsilon}\left[P_{1}\right] \in \Psi_{\mathrm{cl}}^{\text {comp }}$. Therefore, we can define uniquely up to $h^{\infty} \Psi^{-\infty}$ the operators

$$
\begin{gathered}
X_{\varepsilon}=\chi_{\varepsilon}\left[P_{1}\right] \chi_{\varepsilon}\left[P_{2}\right] \in \Psi_{\mathrm{cl}}^{\mathrm{comp}}(M), \psi_{\varepsilon}\left[P_{1}\right] \in \Psi_{\mathrm{cl}}^{-k_{1}}(M), \\
\chi_{\varepsilon}\left[P_{1}\right] \psi_{\varepsilon}\left[P_{2}\right] \in \Psi_{\mathrm{cl}}^{\mathrm{comp}}(M) .
\end{gathered}
$$

By Proposition 2.3, these operators commute with each other and with $P_{1}, P_{2}$ modulo $h^{\infty} \Psi^{-\infty}$. Let $Y$ be any of the operators in (A.7); we will show that it commutes with each $A_{j}$ modulo $h^{\infty} \Psi^{-\infty}$. Take a neighborhood $U$ of $K$ so small that $\left|p_{10}\right|+\left|p_{20}\right| \leq \varepsilon / 4$ on $U$; then, $Y$ is either zero or the identity operator microlocally on $U$. By (L1), decompose $A_{j}=A_{j}^{\prime}+A_{j}^{\prime \prime}$, where $\mathrm{WF}_{h}\left(A_{j}^{\prime}\right) \subset U \times U$ and $A_{j}^{\prime \prime} \in \Psi_{\mathrm{cl}}^{\mathrm{comp}}$. We have $A_{j}=A_{j}^{\prime \prime}$ microlocally away from $U \times U$; therefore, $\left[A_{j}^{\prime \prime}, P_{k}\right]=0$ microlocally near $T^{*} M \backslash U$. By Proposition 2.3, $\left[A_{j}^{\prime \prime}, Y\right]=0$ microlocally near $T^{*} M \backslash U$; therefore, the commutator $\left[A_{j}, Y\right]$ is compactly microlocalized and $\mathrm{WF}_{h}\left(\left[A_{j}, Y\right]\right) \subset U \times U$. However, since $Y=0$ or $Y=I$ microlocally in $U$, we have $\left[A_{j}, Y\right] \in h^{\infty} \Psi^{-\infty}$, as needed.

Since $X_{\varepsilon}=I$ microlocally near $K$ and $\mathrm{WF}_{h}\left(S_{j}\right) \subset K$, we get $(I-$ $\left.X_{\varepsilon}\right) S_{1}, S_{2}\left(I-X_{\varepsilon}\right) \in h^{\infty} \Psi^{-\infty}$. Multiplying (A.6) by $X_{\varepsilon}$, we get for $\varepsilon$ small enough,

$$
\left(X_{\varepsilon} A_{1}\right) P_{1}+\left(X_{\varepsilon} A_{2}\right) P_{2}+S_{1} S_{2}=X_{\varepsilon} \bmod h^{\infty} \Psi^{-\infty} .
$$

Next, by Proposition 2.3

$$
\psi_{\varepsilon}\left[P_{1}\right] P_{1}+\chi_{\varepsilon}\left[P_{1}\right] \psi_{\varepsilon}\left[P_{2}\right] P_{2}=I-X_{\varepsilon} \bmod h^{\infty} \Psi^{-\infty} .
$$

Adding these up, we get

$$
\left(X_{\varepsilon} A_{1}+\psi_{\varepsilon}\left[P_{1}\right]\right) P_{1}+\left(X_{\varepsilon} A_{2}+\chi_{\varepsilon}\left[P_{1}\right] \psi_{\varepsilon}\left[P_{2}\right]\right) P_{2}+S_{1} S_{2}=I+h^{\infty} \Psi^{-\infty} .
$$

The operators $P_{1}, P_{2}, \widetilde{A}_{1}=X_{\varepsilon} A_{1}+\psi_{\varepsilon}\left[P_{1}\right], \widetilde{A}_{2}=X_{\varepsilon} A_{2}+\chi_{\varepsilon}\left[P_{1}\right] \psi_{\varepsilon}\left[P_{2}\right], S_{1}$, $S_{2}$ satisfy the assumptions of Proposition A.2. Applying it, we get the desired spectral result.

\section{A.3. Proof of Proposition A.2 in the General Case}

In this subsection, we prove Proposition A.2 for the general case of $n \geq 2$ operators. For simplicity, we assume that $k_{1}=\cdots=k_{n}=0$; that is, each $P_{j}$ lies in $\Psi^{0}(M)$. (If this is not the case, one needs to replace $L^{2}(M)$ below with certain semiclassical Sobolev spaces).

Let $V$ be the space of all exterior forms on $\mathbb{C}^{n}$; we can represent it as $V_{\text {Even }} \oplus V_{\text {Odd }}$, where

$$
V_{\text {Even }}=\bigoplus_{j \geq 0} \Lambda^{2 j} \mathbb{C}^{n}, V_{\text {Odd }}=\bigoplus_{j \geq 0} \Lambda^{2 j+1} \mathbb{C}^{n}
$$

are the vector spaces of the even and odd degree forms, respectively. Note that $V_{\text {Even }}$ and $V_{\text {Odd }}$ have the same dimension. Define the spaces

$$
L_{\text {Even }}^{2}=L^{2}(M) \otimes V_{\text {Even }}, L_{\text {Odd }}^{2}=L^{2}(M) \otimes V_{\text {Odd }}, L_{V}^{2}=L^{2}(M) \otimes V .
$$


We call elements of $L_{V}^{2}$ forms. They possess properties similar to those of differential forms; beware though that they are not differential forms in our case. We will use the families of operators $\left(A_{j}\right)$ and $\left(P_{j}\right)$ to define the operators

$$
d_{P}, d_{A}^{*}: L_{V}^{2} \rightarrow L_{V}^{2}
$$

given by the formulas

$$
\begin{aligned}
& d_{P}(u \otimes v)=\sum_{j=1}^{n}\left(P_{j} u\right) \otimes\left(e_{j} \wedge v\right), \\
& d_{A}^{*}(u \otimes v)=\sum_{j=1}^{n}\left(A_{j} u\right) \otimes\left(i_{e_{j}} v\right)
\end{aligned}
$$

$u \in L^{2}(M), v \in V$.

Here $e_{1}, \ldots, e_{n}$ is the canonical basis of $\mathbb{C}^{n}$. The notation $i_{e_{j}}$ is used for the interior product by $e_{j}$; this is the adjoint of the operator $v \mapsto e_{j} \wedge v$ with respect to the inner product on $V$ induced by the canonical bilinear inner product on $\mathbb{C}^{n}$. Note that $d_{P}$ and $d_{A}^{*}$ map even forms to odd and vice versa.

A direct calculation shows that under the assumptions (G1)-(G6),

$$
\left(d_{P}+d_{A}^{*}\right)^{2}=I-S_{1} S_{2} \otimes I_{V}+O_{\Psi^{-\infty}}\left(h^{\infty}\right) .
$$

Here $I_{V}$ is the identity operator on $V$, while $I$ is the identity operator on $L_{V}^{2}$. Moreover, since the operators $P_{1}, \ldots, P_{n}$ commute exactly, we have

$$
d_{P}^{2}=0 \text {. }
$$

For $\lambda=\left(\lambda_{1}, \ldots, \lambda_{n}\right) \in \mathbb{C}^{n}$, define the operator

$$
\begin{gathered}
T(\lambda)=\left(\begin{array}{cc}
\left.\left(d_{P-\lambda}+d_{A}^{*}\right)\right|_{L_{\text {Even }}^{2}} & S_{1} \otimes I_{V} \\
S_{2} \otimes I_{V} & 0
\end{array}\right): \mathcal{H}_{1} \rightarrow \mathcal{H}_{2}, \\
\mathcal{H}_{1}=L_{\text {Even }}^{2} \oplus V_{\text {Odd }}, \mathcal{H}_{2}=L_{\text {Odd }}^{2} \oplus V_{\text {Even }} .
\end{gathered}
$$

Here $d_{P-\lambda}$ is defined using the operators $P_{1}-\lambda_{1}, \ldots, P_{n}-\lambda_{n}$ in place of $P_{1}, \ldots, P_{n}$. It follows from (A.11) that for

$$
Q=\left(\begin{array}{cc}
\left.\left(d_{P}+d_{A}^{*}\right)\right|_{L_{\text {Odd }}^{2}} & S_{1} \otimes I_{V} \\
S_{2} \otimes I_{V} & 0
\end{array}\right): \mathcal{H}_{2} \rightarrow \mathcal{H}_{1},
$$

we have $Q T(0)=I+O_{\mathcal{H}_{1} \rightarrow \mathcal{H}_{1}}\left(h^{\infty}\right), T(0) Q=I+O_{\mathcal{H}_{2} \rightarrow \mathcal{H}_{2}}\left(h^{\infty}\right)$. Moreover, it follows from (G1) and (G3) that $\|Q\|_{\mathcal{H}_{2} \rightarrow \mathcal{H}_{1}}=O\left(h^{-r}\right)$. Therefore, for $|\lambda| \leq$ $\delta h^{r}$ and $h$ and $\delta>0$ small enough, the operator $T(\lambda)$ is invertible, with $\left\|T(\lambda)^{-1}\right\|_{\mathcal{H}_{2} \rightarrow \mathcal{H}_{1}}=O\left(h^{-r}\right)$.

Assume that $|\lambda| \leq \delta h^{r}$ and let $\mathbf{1} \in V_{\text {Even }}$ be the basic zero-form on $\mathbb{C}^{n}$. Put $(\alpha(\lambda), v(\lambda))=T(\lambda)^{-1}(0, \mathbf{1})$, where $\alpha(\lambda) \in L_{\text {Even }}^{2}, v(\lambda) \in V_{\text {Odd }}$; then, $(\alpha(\lambda), v(\lambda))$ is the unique solution to the system

$$
\begin{aligned}
\left(d_{P-\lambda}+d_{A}^{*}\right) \alpha(\lambda)+S_{1}(1) \otimes v(\lambda) & =0, \\
\left(S_{2} \otimes I_{V}\right) \alpha(\lambda) & =\mathbf{1} .
\end{aligned}
$$


We further write $v(\lambda)=f(\lambda)+w(\lambda)$, where $f(\lambda)$ is a 1 -form and $w(\lambda)$ is a sum of forms of degree 3 or more. Note that both $f$ and $w$ are holomorphic functions of $\lambda$, with $f(\lambda) \in \mathbb{C}^{n}$.

We claim that $\lambda$ is a joint eigenvalue of $P_{1}, \ldots, P_{n}$ if and only if $f(\lambda)=0$. First of all, if $u$ is a joint eigenfunction, then $T(\lambda)(u \otimes \mathbf{1}, 0)=c(0, \mathbf{1})$ for some scalar $c \neq 0$; therefore, $f(\lambda)=0$ and the joint eigenspace is one dimensional.

Now, assume that $f(\lambda)=0$. We will prove that the solution to (A.13) satisfies $\alpha(\lambda)=u \otimes \mathbf{1}$ for some $u \in L^{2}(M)$; it follows immediately that $\left(P_{1}-\right.$ $\left.\lambda_{1}\right) u=\cdots=\left(P_{n}-\lambda_{n}\right) u=0$. Let $\alpha=u \otimes 1+\beta$, where $\beta$ is a sum of forms of degree 2 or higher. Then, by (A.12),

$$
\left(d_{P-\lambda}+d_{A}^{*}\right)^{2}(u \otimes 1) \in L^{2}(M) \otimes \mathbf{1} .
$$

Next, we get from (A.13)

$$
\left(S_{2} \otimes I_{V}\right)\left(d_{P-\lambda}+d_{A}^{*}\right) \alpha+\left(1+O\left(h^{\infty}\right)\right) v=0 .
$$

The components of this equation corresponding to odd forms of degree 3 or higher depend only on $\beta$ and $w$; therefore, for $h$ small enough, $w=W \beta$ for some operator $W$ of norm $O\left(h^{-r}\right)$. Since $f=0$, we get $v=W \beta$; therefore, by (A.14) and (A.13) multiplied by $d_{P-\lambda}+d_{A}^{*}$,

$$
\left(d_{P-\lambda}+d_{A}^{*}\right)^{2} \beta+\left(d_{P-\lambda}+d_{A}^{*}\right)\left(S_{1}(1) \otimes W \beta\right) \in L^{2}(M) \otimes \mathbf{1} .
$$

Taking the components of this equation corresponding to forms of even degree 2 or higher and recalling (A.11), we get

$$
\left(\left(I-S_{1} S_{2}\right) \otimes I_{V}+O\left(\delta+h^{\infty}\right)\right) \beta=0 .
$$

However, $\left(S_{2} \otimes I_{V}\right) \beta=0$ by (A.13); therefore,

$$
\left(I+O\left(\delta+h^{\infty}\right)\right) \beta=0 .
$$

It follows that $\beta=0$ and the claim is proven.

It remains to show that the equation $f(\lambda)=0$ has exactly one solution in the disk of radius $\delta h^{r}$. For that, we write $Q T(\lambda)=I-R(\lambda)$,

$$
T(\lambda)^{-1}=\left(I+R(\lambda)+(I-R(\lambda))^{-1} R(\lambda)^{2}\right) Q .
$$

We have $Q(\lambda)(0, \mathbf{1})=\left(S_{1}(1) \otimes \mathbf{1}, 0\right)$ and

$$
R(\lambda)=\left(\begin{array}{cc}
\left(d_{P}+d_{A}^{*}\right) d_{\lambda} & 0 \\
\left(S_{2} \otimes I_{V}\right) d_{\lambda} & 0
\end{array}\right)+O\left(h^{\infty}\right) .
$$

Here $d_{\lambda}$ is constructed using $\lambda_{1}, \ldots, \lambda_{n}$ in place of $P_{1}, \ldots, P_{n}$. Now, we use that $R(\lambda)^{2} Q(0, \mathbf{1})=O_{\mathcal{H}_{1}}\left(h^{\infty}\right)$ to conclude that $f(\lambda)=\lambda-g(\lambda ; h)$ with $g=O\left(h^{\infty}\right)$; it then remains to use the contraction mapping principle.

\section{Appendix B. Numerical Results}

\section{B.1. Overview}

This section describes a procedure for computing the quantization symbol $\mathcal{F}(m, l, k)$ from Theorem 1 to an arbitrarily large order in the case

$$
l^{\prime}=l-|k|=O(1)
$$


The reason for the restriction $l^{\prime}=O(1)$ is because then we can use bottom of the well asymptotics for eigenvalues of the angular operator; otherwise, we would have to deal with nondegenerate trajectories, quantization conditions for which are harder to compute numerically; see for example [14].

We first use the Eq. (1.17); once we get rid of the semiclassical parameter $h$ (remembering that the original problem was $h$-independent), the number $\omega=\mathcal{F}(m, l, k)$ is the solution to the equation

$$
\mathcal{G}^{r}(m, \omega, k)=\mathcal{G}^{\theta}\left(l^{\prime}, \omega, k\right) .
$$

Here $\mathcal{G}^{r}, \mathcal{G}^{\theta}$ are the nonsemiclassical analogs of $\mathcal{F}^{r}, \mathcal{F}^{\theta}$; namely, (1.14) and (1.16) take the form

$$
\begin{aligned}
\lambda=\mathcal{G}^{r}(m, \omega, k) & \sim \sum_{j \geq 0} \mathcal{G}_{j}^{r}(m, \omega, k), \\
\lambda=\mathcal{G}^{\theta}\left(l^{\prime}, \omega, k\right) & \sim \sum_{j \geq 0} \mathcal{G}_{j}^{\theta}\left(l^{\prime}, \omega, k\right),
\end{aligned}
$$

respectively. The functions $\mathcal{G}_{j}^{r}, \mathcal{G}_{j}^{\theta}$ are homogeneous of degree $2-j$ in the following sense:

$$
\mathcal{G}_{j}^{r}\left(m, M_{s} \omega, s k\right)=s^{2-j} \mathcal{G}_{j}^{r}(m, \omega, k), \mathcal{G}_{j}^{\theta}\left(l^{\prime}, M_{s} \omega, s k\right)=s^{2-j} \mathcal{G}_{j}^{\theta}\left(l^{\prime}, \omega, k\right), s>0 .
$$

Here $M_{s} \omega=s \operatorname{Re} \omega+i \operatorname{Im} \omega$; the lack of dilation in the imaginary part of $\omega$ reflects the fact that it is very close to the real axis.

We will describe how to compute $\mathcal{G}_{j}^{r}, \mathcal{G}_{j}^{\theta}$ for an arbitrary value of $j$ in Sect. B.3. The method is based on a quantization condition for barrier-top resonances, studied in Sect. 4.3; their computation is explained in Sect. B.2 and a MATLAB implementation and data files for several first QNMs can be found online at http://math.berkeley.edu/ dyatlov/qnmskds. We explain why the presented method gives the quantization conditions of Propositions 1.5 and 1.6, but we do not provide a rigorous proof.

We now compare the pseudopoles given by quantization conditions to QNMs for the Kerr metric ${ }^{10}$ computed by the authors of [6] using Leaver's continued fraction method-see [6, Section 4.6] for an overview of the method and [7, Appendix E] and [8, Section IV] for more details. The QNM data for the case of scalar perturbations, studied in this paper, computed using Leaver's method can be found online at http://www.phy.olemiss.edu/ berti/ qnms.html.

Figure 5 compares the second order approximation to QNMs (that is, solution to the Eq. (B.2) constructed using $\mathcal{G}_{j}^{r}$ and $\mathcal{G}_{j}^{\theta}$ for $j \leq 2$ ) to the QNMs of [6]. Each branch on the picture shows the trajectory of the QNM with fixed parameters $m, l, k$ for $a \in[0,0.25]$; the marked points correspond

10 The results of the present paper do not apply to the Kerr case $\Lambda=0$, due to lack of control on the scattering resolvent at the asymptotically flat spatial infinity. However, the resonances described by $(0.2)$ are generated by trapping, which is located in a compact set; therefore, we can still make sense of the quantization condition and compute approximate QNMs. 

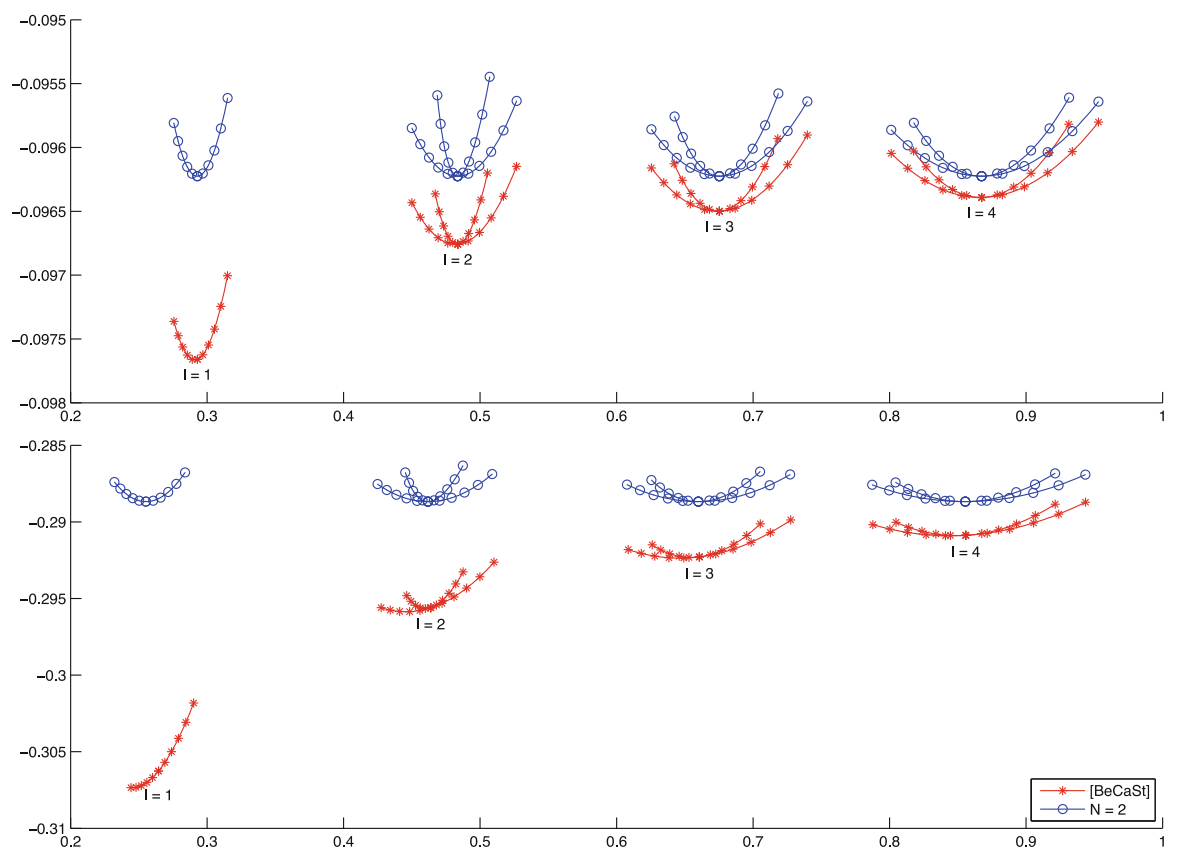

Figure 5. Comparison of order 2 approximation to QNMs with the data of [6]. Here $l=1, \ldots, 4, k=-l,-l+1, l-1, l$ (left to right), and $m=0$ (top) and 1 (bottom)

to $a=0,0.05, \ldots, 0.25$. The branches for same $m, l$ and different $k$ converge to the Schwarzschild QNMs as $a \rightarrow 0$. We see that the approximation gets better when $l$ increases, but worse if one increases $m$; this agrees well with the fact that the computed quantization conditions are expected to work when $l$ is large and $m$ is bounded.

The left part of Fig. 6 compares the second and fourth order approximations with the QNMs of [6] (with the same values of $a$ as before); we see that the fourth order approximation is considerably more accurate than the second order one, and the former is more accurate for a smaller value of $l^{\prime}$. Finally, the right part of Fig. 6 is a log-log plot of the error of approximations of degree $1-4$, as a function of $l$; we see that the error decreases polynomially in $l$.

\section{B.2. Barrier-Top Resonances}

Here we study a general spectral problem to which we will reduce both the radial and the angular problems in the next subsection. Our computation is based on the following observation: when the quantization condition of Sect. 4.3 is satisfied, the function $u_{+}$has the microlocal form (4.3), with the symbol behaving like $\left(r-r_{0}\right)^{m}$ near the trapped set. This can be seen from the proof of Proposition 4.6: if $\beta=-i h m$, then $\tilde{u}_{ \pm}^{+}(x)=x^{m}$ and $B_{1} \tilde{u}_{ \pm}^{+}$has to have the form (4.3). The calculations below are similar to [21, Section 3]. 

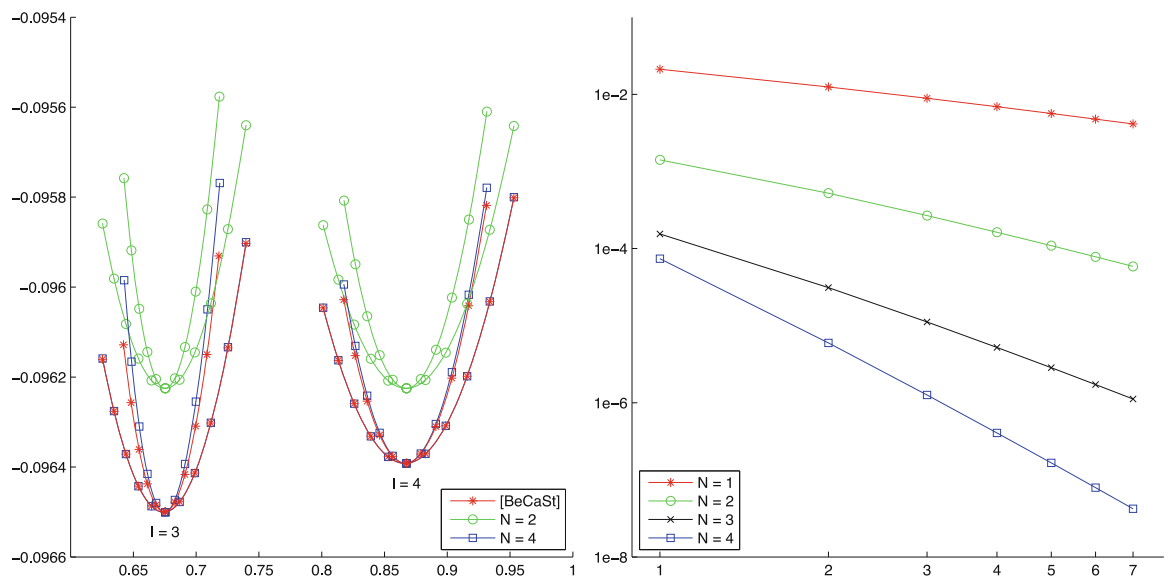

Figure 6. Left comparison of order 2 and 4 approximations to QNMs with the data of [6]. Here $l=3,4, l^{\prime}=0,1$, and $m=0$. Right $\log -\log$ plot of the error of order 1-4 approximations to QNMs, as compared to [6]. Here $a=0.1, k=l$, $m=0$, and $l$, plotted on the $x$ axis, ranges from 1 to 7

Consider the operator

$$
P_{y}=D_{y} A(y) D_{y}+B(y ; \omega, k) .
$$

Here the function $A(y)$ is independent of $\omega, k$, real-valued, and $A(0)>0 ; B$ is a symbol of order 2 :

$$
B(y ; \omega, k) \sim \sum_{j \geq 0} B_{j}(y ; \omega, k),
$$

with $B_{j}$ homogeneous of degree $2-j$ in the sense of (B.3). We also require that $B_{0}$ be real-valued and

$$
B_{0}^{\prime}(0 ; \omega, k)=0, B_{0}^{\prime \prime}(0 ; \omega, k)<0 .
$$

We will describe an algorithm to find the quantization condition for eigenvalues $\lambda$ of $P_{y}$ with eigenfunctions having the outgoing WKB form (B.6) near $y=0$; we will compute $\lambda$ as a symbol of order 2 :

$$
\lambda \sim \sum_{j \geq 0} \lambda_{j}(\omega, k), \lambda_{j}\left(M_{s} \omega, s k\right)=s^{2-j} \lambda(\omega, k), s>0 .
$$

More precisely, we will show how to inductively compute each $\lambda_{j}$. The principal part $\lambda_{0}$ is given by the following barrier-top condition:

$$
\lambda_{0}=B_{0}(0 ; \omega, k) .
$$

In this case, we have

$$
B_{0}(y ; \omega, k)=\lambda_{0}(\omega, k)-y^{2} U_{0}(y ; \omega, k),
$$


where $U_{0}$ is a smooth function, and $U_{0}(0)=-V^{\prime \prime}(0) / 2>0$. Define the phase function $\psi_{0}(y ; \omega, k)$ such that

$$
\psi_{0}^{\prime}(y ; \omega, k)=y \sqrt{U_{0}(y ; \omega ; k) / A(y)} ;
$$

note that $\psi_{0}$ is homogeneous of degree 1 . We will look for eigenfunctions of the WKB form

$$
u(y ; \omega, k)=e^{i \psi_{0}(y ; \omega, k)} a(y ; \omega, k),
$$

solving the equation $P_{y} u=\lambda u$ up to $O(|\omega|+|k|)^{-\infty}$ error near $y=0$. Here $a$ is a symbol of order zero:

$$
a(y ; \omega, k) \sim \sum a_{j}(y ; \omega, k)
$$

with $a_{j}$ homogeneous of order $-j$.

Substituting (B.6) into the equation $P_{y} u=\lambda u$ and gathering terms with the same degree of homogeneity, we get the following system of transport equations:

$$
\begin{gathered}
\left(L_{0}-B_{1}+\lambda_{1}\right) a_{j}=-L_{1} a_{j-1}+\sum_{0<l \leq j}\left(B_{l+1}-\lambda_{l+1}\right) a_{j-l}, j \geq 0 \\
L_{0}=2 i \psi_{0}^{\prime} A \partial_{y}+i\left(A \psi_{0}^{\prime}\right)^{\prime}=2 i \sqrt{U_{0}(y) A(y)} y \partial_{y}+i\left(y \sqrt{U_{0}(y) A(y)}\right)^{\prime} \\
L_{1}=\partial_{y} A(y) \partial_{y}
\end{gathered}
$$

with the convention $a_{-1}=0$.

Now, consider the space of infinite sequences

$$
\mathbb{C}^{\infty}=\left\{\mathbf{a}=\left(\mathbf{a}^{j}\right)_{j=0}^{\infty} \mid \mathbf{a}^{j} \in \mathbb{C}\right\}
$$

and the operator $T: C^{\infty}(\mathbb{R}) \rightarrow \mathbb{C}^{\infty}$ defined by

$$
T(a)=\mathbf{a}, \quad \mathbf{a}_{j}=\partial_{y}^{j} a(0) / j ! .
$$

Let the operators $\mathbf{L}_{j}, \mathbf{B}_{j}: \mathbb{C}^{\infty} \rightarrow \mathbb{C}^{\infty}$ be defined by the relations

$$
T L_{j}=\mathbf{L}_{j} T, \quad T B_{j}=\mathbf{B}_{j} T .
$$

We treat $\mathbf{L}_{j}, \mathbf{B}_{j}$ as infinite dimensional matrices. We see that each $\mathbf{B}_{j}$ is lower triangular, with elements on the diagonal given by $B_{j}(0) ;\left(\mathbf{L}_{1}\right)_{j k}=0$ for $j+2<k$. As for $\mathbf{L}_{0}$, due to the factor $y$ in front of the differentiation it is lower triangular and

$$
\left(\mathbf{L}_{0}\right)_{j j}=i(2 j+1) \sqrt{U_{0}(0) A(0)} .
$$

One can show that there exists a smooth nonzero function $a_{0}$ solving $\left(L_{0}-\right.$ $\left.B_{1}+\lambda_{1}\right) a_{0}=0$ if and only if one of the diagonal elements of the matrix $\mathbf{L}_{0}-\mathbf{B}_{1}+\lambda_{1}$ is zero (the kernel of this matrix being spanned by $T a_{0}$ ). Let $m \geq 0$ be the index of this diagonal element; this will be a parameter of the quantization condition. We can now find

$$
\lambda_{1}=B_{1}(0)-i(2 m+1) \sqrt{U_{0}(0) A(0)} .
$$

Now, there exists a nonzero functional $\mathbf{f}$ on $\mathbb{C}^{\infty}$, such that $\mathbf{f}(\mathbf{a})$ depends only on $\mathbf{a}^{0}, \ldots, \mathbf{a}^{m}$, and $\mathbf{f}$ vanishes on the image of $\mathbf{L}_{0}-\mathbf{B}_{1}+\lambda_{1}$. Moreover, one 
can show that the equation $\left(L_{0}-B_{1}+\lambda_{1}\right) a=b$ has a smooth solution $a$ if and only if $\mathbf{f}(T b)=0$.

Take $a_{0}$ to be a nonzero element of the kernel of $L_{0}-B_{1}+\lambda_{1}$; we normalize it so that $\mathbf{f}\left(T a_{0}\right)=0$. Put $\mathbf{a}_{j}=T a_{j}$; then, the transport equations become

$$
\left(\mathbf{L}_{0}-\mathbf{B}_{1}+\lambda_{1}\right) \mathbf{a}_{j}=-\mathbf{L}_{1} \mathbf{a}_{j-1}+\sum_{0<l \leq j}\left(\mathbf{B}_{l+1}-\lambda_{l+1}\right) \mathbf{a}_{j-l}, \quad j>0 .
$$

We normalize each $a_{j}$ so that $\mathbf{f}\left(\mathbf{a}_{j}\right)=0$ for $j>0$. The $j$ th transport equation has a solution if and only if the $\mathbf{f}$ kills the right-hand side, which makes it possible to find

$$
\lambda_{j+1}=\mathbf{f}\left(-\mathbf{L}_{1} a_{j-1}+\sum_{0<l<j} \mathbf{B}_{l+1} \mathbf{a}_{j-l}\right), \quad j>0 .
$$

Using the Eqs. (B.5), (B.8), (B.10), and (B.9), we can find all $\lambda_{j}$ and $\mathbf{a}_{j}$ inductively.

\section{B.3. Radial and Angular Quantization Conditions}

We start with the radial quantization condition. Consider the original radial operator

$$
\begin{aligned}
& P_{r}=D_{r}\left(\Delta_{r} D_{r}\right)+V_{r}(r ; \omega, k), \\
& V_{r}(r ; \omega, k)=-\Delta_{r}^{-1}(1+\alpha)^{2}\left(\left(r^{2}+a^{2}\right) \omega-a k\right)^{2} .
\end{aligned}
$$

It has the form (B.4), with

$$
y=r-r_{0}, A(y)=\Delta_{r}, B(y ; \omega, k)=V_{r}(r ; \omega, k) .
$$

Here $r_{0}$ is the point where $V_{r}$ achieves its maximal value, corresponding to the trapped point $x_{0}$ in Sect. 4.1. Now the previous subsection applies, with the use of the outgoing microlocalization mentioned in the beginning of that subsection. Using (B.5) and (B.8), we can compute near $a=0$,

$$
\begin{gathered}
r_{0}=3 M_{0}-\frac{2 a k\left(1-9 \Lambda M_{0}^{2}\right)}{9 M_{0} \operatorname{Re} \omega}+O\left(a^{2}\left(|k|^{2}+|\omega|^{2}\right)\right), \\
\mathcal{G}_{0}^{r}=\frac{27 M_{0}^{2}}{1-9 \Lambda M_{0}^{2}}\left(1-\frac{2 a k}{9 M_{0}^{2} \operatorname{Re} \omega}\right)(\operatorname{Re} \omega)^{2}+O\left(a^{2}\left(|k|^{2}+|\omega|^{2}\right)\right), \\
\mathcal{G}_{0}^{r}+\mathcal{G}_{1}^{r}=\left[i(m+1 / 2)+\frac{3 \sqrt{3} M_{0} \omega}{\sqrt{1-9 \Lambda M_{0}^{2}}}\right]^{2}+O(1) \quad \text { for } a=0
\end{gathered}
$$

reintroducing the semiclassical parameter, we get the formulas for $\mathcal{F}^{r}$ in Proposition 1.5.

Now, we consider the angular problem. Without loss of generality, we assume that $k>0$. After the change of variables $y=\cos \theta$, the operator $\left.P_{\theta}\right|_{\mathcal{D}_{k}^{\prime}}$ takes the form

$$
P_{y}=D_{y}\left(1-y^{2}\right)\left(1+\alpha y^{2}\right) D_{y}+\frac{(1+\alpha)^{2}\left(a \omega\left(1-y^{2}\right)-k\right)^{2}}{\left(1-y^{2}\right)\left(1+\alpha y^{2}\right)} .
$$

We are now interested in the bottom of the well asymptotics for the eigenvalues of $P_{y}$, with the parameter $l^{\prime}$ from (B.1) playing the role of the quantization 
parameter $m$. The critical point for the principal symbol of the operator $P_{y}$ is $(0,0)$. To reduce the bottom of the well problem to the barrier-top problem, we formally rescale in the complex plane, introducing the parameter $y^{\prime}=e^{i \pi / 4} y$, so that $\left(y^{\prime}\right)^{2}=i y^{2}$. We do not provide a rigorous justification for such an operation; we only note that the WKB solution of (B.6) looks like $e^{i c\left(y^{\prime}\right)^{2}} a=e^{-c y^{2}} a$ near $y=0$ for some positive constant $c$; therefore, it is exponentially decaying away from the origin, reminding one of the exponentially decaying Gaussians featured in the bottom of the well asymptotics (see for example [21, Section 3] or the discussion following [41, Proposition 4.3]). There is a similar calculation of the bottom of the well resonances based on quantum Birkhoff normal form; see for example [15]. The rescaled operator $P_{y^{\prime}}=-i P_{y}$ takes the form (B.4), with $y^{\prime}$ taking the place of $y$ and

$$
\begin{aligned}
A\left(y^{\prime}\right) & =\left(1+i\left(y^{\prime}\right)^{2}\right)\left(1-i \alpha\left(y^{\prime}\right)^{2}\right), B\left(y^{\prime} ; \omega, k\right) \\
& =-\frac{i(1+\alpha)^{2}\left(a \omega\left(1+i\left(y^{\prime}\right)^{2}\right)-k\right)^{2}}{\left(1+i\left(y^{\prime}\right)^{2}\right)\left(1-i \alpha\left(y^{\prime}\right)^{2}\right)} .
\end{aligned}
$$

We can now formally apply the results of Sect. B.2; note that, even though $A$ and $B$ are not real-valued, we have

$$
A(0)=1, B_{0}(0)=-i(1+\alpha)^{2}(a \operatorname{Re} \omega-k)^{2}, B_{0}^{\prime \prime}(0)<0 .
$$

An interesting note is that when $a=0$ and $k>0$, the process described in Sect. B.2 gives the spherical harmonics $\lambda=l(l+1)$ exactly and without the assumption (B.1). In fact, the first three terms of the asymptotic expansion of $\lambda$ sum to $l(l+1)$ and the remaining terms are zero.

\section{References}

[1] Abbott, B.P., et al.: The LIGO scientic collaboration, search for gravitational wave ringdowns from perturbed black holes in LIGO S4 data. Phys. Rev. D 80, 062001 (2009)

[2] Andersson, L., Blue, P.: Hidden symmetries and decay for the wave equation on the Kerr spacetime. Preprint, arXiv:0908.2265

[3] Bachelot, A.: Gravitational scattering of electromagnetic field by Schwarzschild black hole. Ann. Inst. H. Poincaré Phys. Théor. 54, 261-320 (1991)

[4] Bachelot, A.: Scattering of electromagnetic field by de Sitter-Schwarzschild black hole. Non-linear hyperbolic equations and field theory. Pitman Res. Notes Math. Ser. 253, 23-35

[5] Bachelot, A., Motet-Bachelot, A.: Les résonances d'un trou noir de Schwarzschild. Ann. Inst. H. Poincaré Phys. Théor. 59, 3-68 (1993)

[6] Berti, E., Cardoso, V., Starinets, A.: Quasinormal modes of black holes and black branes. Class. Quant. Grav. 26, 163001 (2009)

[7] Berti, E., Cardoso, V., Will, C.M.: On gravitational-wave spectroscopy of massive black holes with the space interferometer LISA. Phys. Rev. D 73, 064030 (2006) 
[8] Berti, E., Kokkotas, K.: Quasinormal modes of Kerr-Newman black holes: coupling of electromagnetic and gravitational perturbations. Phys. Rev. D 71, 124008 (2005)

[9] Blue, P., Sterbenz, J.: Uniform decay of local energy and the semi-linear wave equation on Schwarzschild space. Comm. Math. Phys. 268, 481-504 (2006)

[10] Bony, J.-F., Häfner, D.: Decay and non-decay of the local energy for the wave equation on the de Sitter-Schwarzschild metric. Comm. Math. Phys. 282, 697-719 (2008)

[11] Chandrasekhar, S.: The Mathematical Theory of Black Holes. Oxford Classic Texts in the Physical Sciences. Oxford University Press, Oxford (2000)

[12] Charbonnel, A.-M.: Spectre conjoint d'opérateurs pseudodifférentiels qui commutent. Ann. Fac. Sci. Toulouse Math. 5, 109-147 (1983)

[13] Christiansen, T., Zworski, M.: Resonance wave expansions: two hyperbolic examples. Comm. Math. Phys. 212, 323-336 (2000)

[14] Colinde Verdière, Y.: Bohr-Sommerfeld rules to all orders. Ann. Henri Poincaré 6, 925-936 (2005)

[15] Colin de Verdière, Y., Guillemin, V.: A semi-classical inverse problem I: Taylor expansions. Preprint, arXiv:0802.1605

[16] Colinde Verdière, Y., Parisse, B.: Équilibre instable en régime semi-classique: I-Concentration microlocale. Comm. Partial Differ. Equ. 19, 1535-1563 (1994)

[17] Dafermos, M., Rodnianski, I.: The wave equation on Schwarzschild-de Sitter spacetimes. Preprint, arXiv:0709.2766

[18] Dafermos, M., Rodnianski, I.: Lectures on black holes and linear waves. Preprint, arXiv:0811.0354v1

[19] Dafermos, M., Rodnianski, I.: Decay for solutions of the wave equation on Kerr exterior space-times I-II: the cases of $|a| \ll M$ or axisymmetry. Preprint, arXiv:1010.5132

[20] Datchev, K., Vasy, A.: Gluing semiclassical resolvent estimates via propagation of singularities. Preprint, arXiv:1008.3964

[21] Dimassi, M., Sjöstrand, J.: Spectral Asymptotics in the Semi-classical Limit. Cambridge University Press, Cambridge (1999)

[22] Donninger, R., Schlag, W., Soffer, A.: A proof of Price's Law on Schwarzschild black hole manifolds for all angular momenta. Preprint, arXiv:0908.4292

[23] Donninger, R., Schlag, W., Soffer, A.: On pointwise decay of linear waves on a Schwarzschild black hole background. Preprint, arXiv:0911.3179

[24] Duistermaat, J.J.: On global action-angle coordinates. Comm. Pure Appl. Math. 33, 687-706 (1980)

[25] Dunford, N., Schwarz, J.T.: Linear Operators, Part I: General Theory. Interscience, New York (1958)

[26] Dyatlov, S.: Quasi-normal modes and exponential energy decay for the Kerr-de Sitter black hole. Comm. Math. Phys. 306, 119-163 (2011)

[27] Dyatlov, S.: Exponential energy decay for Kerr-de Sitter black holes beyond event horizons. Math. Res. Lett. (to appear), arXiv:1010.5201

[28] Finster, F., Kamran, N., Smoller, J., Yau, S.-T.: Decay of solutions of the wave equation in the Kerr geometry. Comm. Math. Phys. 264, 465-503 (2006) 
[29] Finster, F., Kamran, N., Smoller, J., Yau, S.-T.: Erratum: decay of solutions of the wave equation in the Kerr geometry. Comm. Math. Phys. 280, 563-573 (2008)

[30] Guillemin, V., Sternberg, S.: Semi-classical analysis. Lecture Notes (version

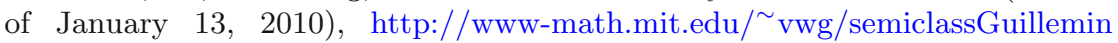
Sternberg.pdf

[31] Hitrik, M., Sjöstrand, J.: Non-selfadjoint perturbations of selfadjoint operators in two dimensions. I. Ann. Henri Poincaré 5, 1-73 (2004)

[32] Hitrik, M., Sjöstrand, J., SanVũ Ngọc, : Diophantine tori and spectral asymptotics for nonselfadjoint operators. Am. J. Math. 129, 105-182 (2007)

[33] Horowitz, G.T., Hubeny, V.E.: Quasinormal modes of AdS black holes and the approach to thermal equilibrium. Phys. Rev. D 62, 024027 (2000)

[34] Hörmander, L.: The Analysis of Linear Partial Differential Operators. I. Distribution Theory and Fourier Analysis. Springer, Berlin (1990)

[35] Kokkotas, K.D., Schmidt, B.G.: Quasi-normal modes of stars and black holes. Living Rev. Relativ. 2 (1999), http://www.livingreviews.org/lrr-1999-2

[36] Konoplya, R.A., Zhidenko, A.: High overtones of Schwarzschild-de Sitter quasinormal spectrum. JHEP 0406, 037 (2004)

[37] Konoplya, R.A., Zhidenko, A.: Decay of a charged scalar and Dirac fields in the Kerr-Newman-de Sitter background. Phys. Rev. D 76, 084018 (2007)

[38] Melrose, R., Sá Barreto, A., Vasy, A.: Asymptotics of solutions of the wave equation on de Sitter-Schwarzschild space. Preprint, arXiv:0811.2229

[39] Pravica, D.: Top resonances of a black hole. R. Soc. Lond. Proc. Ser. A 455, 3003-3018 (1999)

[40] Ramond, T.: Semiclassical study of quantum scattering on the line. Comm. Math. Phys. 177, 221-254 (1996)

[41] Sá Barreto, A., Zworski, M.: Distribution of resonances for spherical black holes. Math. Res. Lett. 4, 103-121 (1997)

[42] Sjöstrand, J.: Semiclassical resonances generated by nondegenerate critical points. Pseudodifferential operators, Lecture Notes in Mathematics, vol. 1256, pp. 402-429. Springer, Berlin (1987)

[43] Sjöstrand, J.: Semi-excited states in nondegenerate potential wells. Asymp. Anal. 6, 29-43 (1992)

[44] Sjöstrand, J., Zworski, M.: Quantum monodromy and semi-classical trace formulae. J. Math. Pures Appl. 81, 1-33 (2002)

[45] Tang, S.-H., Zworski, M.: Resonance expansions of scattered waves. Comm. Pure Appl. Math. 53, 1305-1334 (2000)

[46] Tataru, D.: Local decay of waves on asymptotically flat stationary space-times. Preprint, arXiv:0910.5290

[47] Tataru, D., Tohaneanu, M.: Local energy estimate on Kerr black hole backgrounds. Preprint, arXiv:0810.5766

[48] Taylor, M.: Partial Differential Equations, I. Basic Theory. Springer, Berlin (1996)

[49] Tohaneanu, M.: Strichartz estimates on Kerr black hole backgrounds. Preprint, arXiv:0910.1545 
[50] Vasy, A.: Microlocal analysis of asymptotically hyperbolic and Kerr-de Sitter spaces. Preprint, arXiv:1012.4391

[51] Vũ Ngọc, S.: Systèmes intégrables semi-classiques: du local au global. Société Mathématique de France, France (2006)

[52] Wunsch, J., Zworski, M.: Resolvent estimates for normally hyperbolic trapped sets. Preprint, arXiv:1003.4640

[53] Yoshida, S., Uchikata, N., Futamase, T.: Quasinormal modes of Kerr-de Sitter black holes. Phys. Rev. D 81, 044005 (2010)

[54] Zworski, M.: Semiclassical analysis. Graduate Studies in Mathematics. AMS (2012), http://math.berkeley.edu/ $\sim_{\text {zworski/semiclassical.pdf }}$

Semyon Dyatlov

Department of Mathematics

University of California

Evans Hall

Berkeley

CA 94720

USA

e-mail: dyatlov@math. berkeley.edu

Communicated by Piotr T. Chrusciel.

Received: April 29, 2011.

Accepted: December 6, 2011. 